Aus der Klinik für Augenheilkunde

(Prof. Dr. med. H. Hoerauf)

der Medizinischen Fakultät der Universität Göttingen

\title{
Einfluss der makulären Ischämie auf funktionelle und morphologische Parameter bei Patienten mit diabetischer Retinopathie
}

\author{
INAUGURAL-DISSERTATION \\ zur Erlangung des Doktorgrades \\ der Medizinischen Fakultät der \\ Georg-August-Universität zu Göttingen
}

vorgelegt von

Greta Zinser

aus

Paderborn

Göttingen 2020 


\section{Betreuungsausschuss}

Betreuer:

Prof. Dr. med. N. Feltgen

Ko-Betreuer:

Prof. Dr. med. D. Raddatz

\section{Prüfungskommission}

Referent/in

Ko-Referent/in:

Promoter-Vertreterin:

Datum der mündlichen Prüfung:
Prof. Dr. med. N. Feltgen

Prof. Dr. med. D. Raddatz

Prof. Dr. med. M. Schön

04.08.2021 
Hiermit erkläre ich, die Dissertation mit dem Titel "Einfluss der makulären Ischämie auf funktionelle und morphologische Parameter bei Patienten mit diabetischer Retinopathie" eigenständig angefertigt und keine anderen als die von mir angegebenen Quellen und Hilfsmittel verwendet zu haben.

Göttingen, den 


\section{Inhaltsverzeichnis}

Abbildungsverzeichnis.

Tabellenverzeichnis II

Abkürzungsverzeichnis. .III

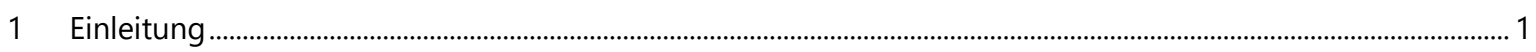

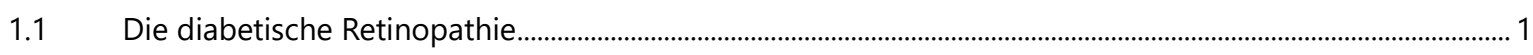

1.1.1 Terminologie und Klassifikation ........................................................................................................

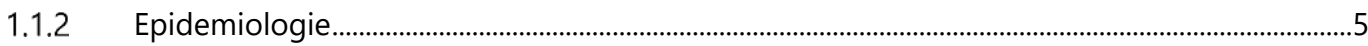

1.1.3 Pathophysiologie ......................................................................................................................

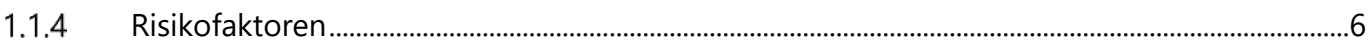

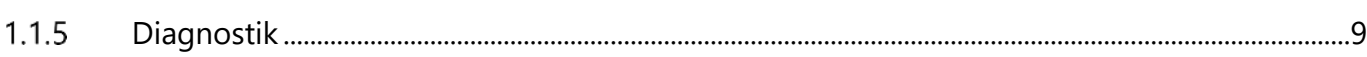

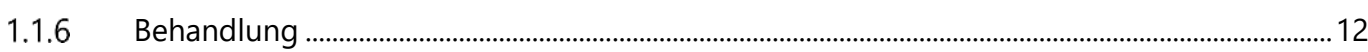

$1.2 \quad$ Zielsetzung und wissenschaftlicher Hintergrund ............................................................................................. 15

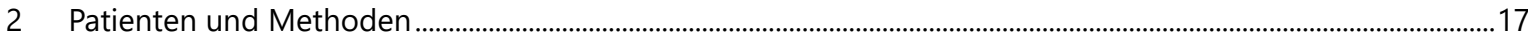

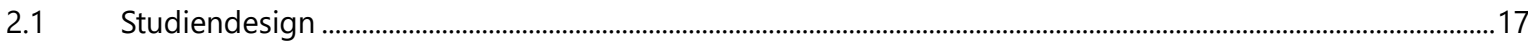

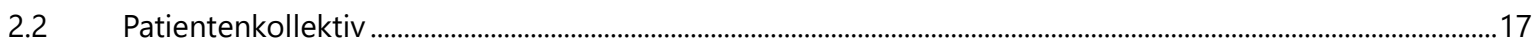

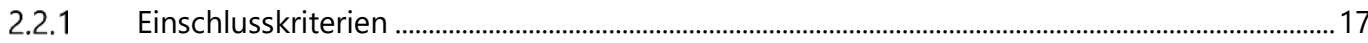

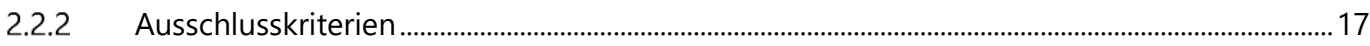

2.3 Untersuchungen, Datenerhebung und -verarbeitung …............................................................................18

2.3.1 Krankheitsspezifische Anamnese ......................................................................................................... 19

2.3.2 Sphärisches Äquivalent.................................................................................................................2 20

2.3.3 Visus- und Kontrastvisusbestimmung.............................................................................................20

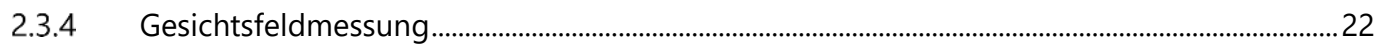

2.3.5 Optische Kohärenztomografie (OCT) .......................................................................................23

2.3.6 Fundusfotografie und Fluoreszeinangiografie ............................................................................25

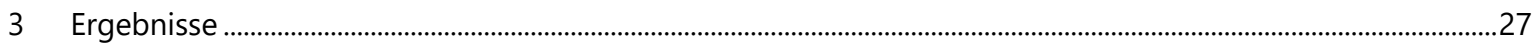

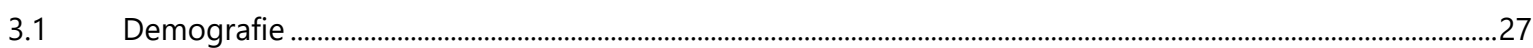

3.2 Fundusfotografie und Fluoreszeinangiografie .................................................................................................3

3.3 Sphärisches Äquivalent ...........................................................................................................................................32

3.4 Visus- und Kontrastvisusbestimmung .................................................................................................................

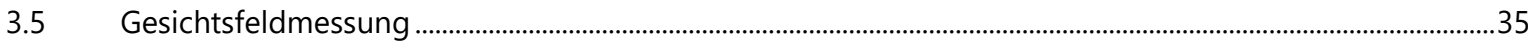

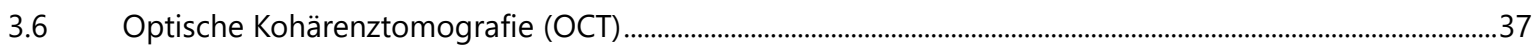

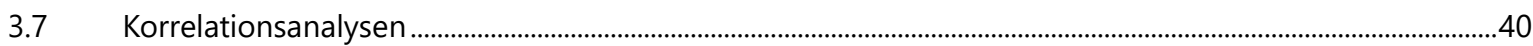

3.7.1 Ischämiefläche vs. demografische Daten ........................................................................................40 


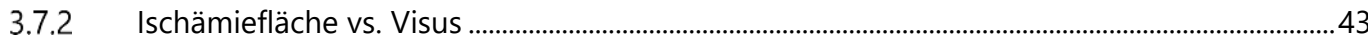

3.7.3 Ischämiefläche vs. perimetrische Daten ………………………………...............................................45

3.7.4 Ischämiefläche vs. OCT-Ergebnisse ...............................................................................................4

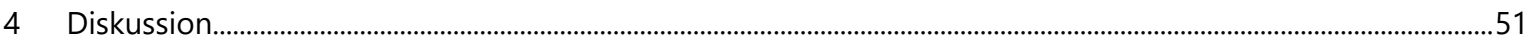

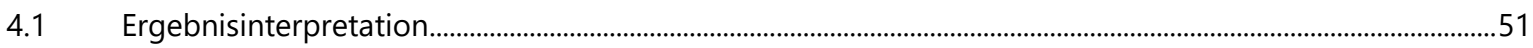

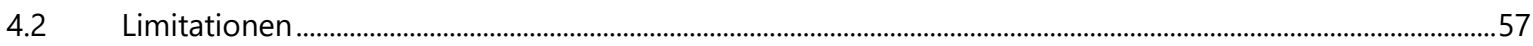

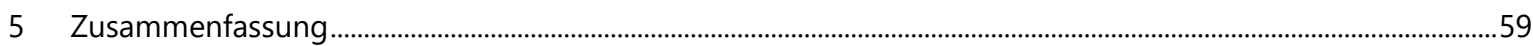

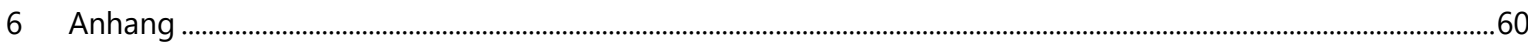

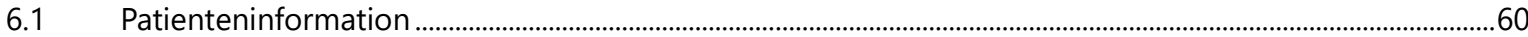

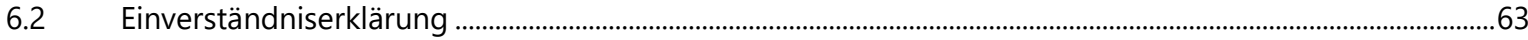

6.3 Untersuchungsbögen ..................................................................................................................................66

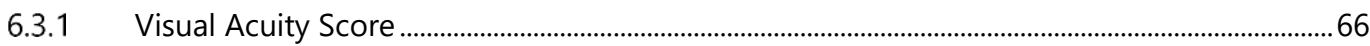

6.3.2 Bailey-Lovie-Chart ............................................................................................................................. 67

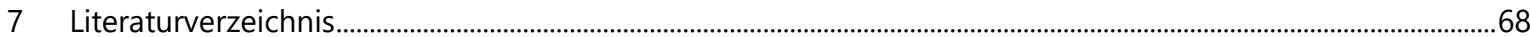




\section{Abbildungsverzeichnis}

Abbildung 1: Foveale avaskuläre Zone in der Fluoreszeinangiografie, Normalbefund ................................................. 2

Abbildung 2: Diabetische Makulopathie mit Einblutungen, harten Exsudaten und Makulaödem .............................. 3

Abbildung 3: Schweregradeinteilung der diabetischen Retinopathie anhand der ETDRS-Skala................................. 5

Abbildung 4: Berechnung des sphärischen Äquivalents ................................................................................................... 9

Abbildung 5: Darstellung der Fovea und Retina mittels OCT im Querschnitt .............................................................11

Abbildung 6: $50^{\circ}$ Fundusaufnahme einer gesunden Netzhaut .........................................................................................12

Abbildung 7: Mittlere Phase einer Fluoreszeinangiografie einer gesunden Netzhaut, 30-Ausschnitt ...................12

Abbildung 8: Behandlung der diabetischen Retinopathie .................................................................................................14

Abbildung 9: Mittlere Phase einer Fluoreszeinangiografie einer gesunden Netzhaut und normwertiger FAZ .....15

Abbildung 10: Mittlere Phase einer Fluoreszeinangiografie bei diabetischer Makulopathie......................................15

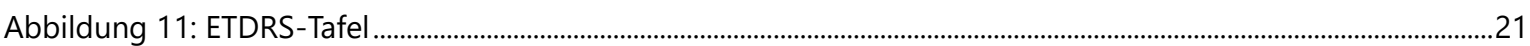

Abbildung 12: Beispiel einer perimetrischen Untersuchung mittels Octopus 900 ....................................................22

Abbildung 13: Beispiele Defektstärken für geringen, mäßigen und ausgeprägten Defekt ........................................22

Abbildung 14: Bereiche der OCT-Messung, Schema......................................................................................................24

Abbildung 15: Beispiel einer OCT-Messung in Absolutwerten- und Graustufendarstellung .....................................24

Abbildung 16: Beispiel einer Abmessungsbestimmung ..............................................................................................................26

Abbildung 17: Geschlechtergetrennte Verteilung der Diabetes-Typen...........................................................................28

Abbildung 18: Gemessene Ischämieflächen $\left[\mathrm{mm}^{2}\right]$ von U1 und U2 anhand des Fluoreszeinangiografie-Bildes.30 Abbildung 19: Übersicht über die gemessen Ischämieflächen $\left[\mathrm{mm}^{2}\right]$ im Fluoreszeinangiografie-Bild ...................31

Abbildung 20: Mittelwerte der gewerteten Fangfragen in \% ..........................................................................................36

Abbildung 21: Analyse-übergreifende Darstellung von mean center subfield thickness $[\mu \mathrm{m}]$ und mean center

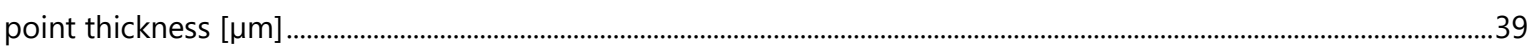

Abbildung 22: Analyse-übergreifende Darstellung des mean total macular volume $\left[\mathrm{mm}^{3}\right]$....................................39

Abbildung 23: Gemittelte Ischämiefläche $\left[\mathrm{mm}^{2}\right]$ vs. Patientenalter [a] ..........................................................................40

Abbildung 24: Gemittelte Ischämiefläche $\left[\mathrm{mm}^{2}\right]$ vs. HbA1c [\%] ......................................................................................41

Abbildung 25: Gemittelte Ischämiefläche $\left[\mathrm{mm}^{2}\right]$ vs. Erkrankungsdauer [a] ..................................................................41

Abbildung 26: Gemittelte Ischämiefläche $\left[\mathrm{mm}^{2}\right]$ vs. Therapiedauer mit Insulin [a] ...................................................42

Abbildung 27: Gemittelte Ischämiefläche [ $\mathrm{mm}^{2}$ ] vs. ETDRS Visual Acuity Score...........................................................43

Abbildung 28: Gemittelte Ischämiefläche $\left[\mathrm{mm}^{2}\right]$ vs. high-contrast Visus ......................................................................4

Abbildung 29: Gemittelte Ischämiefläche $\left[\mathrm{mm}^{2}\right]$ vs. low-contrast Visus.......................................................................4

Abbildung 30: Gemittelte Ischämiefläche $\left[\mathrm{mm}^{2}\right]$ vs. mean sensitivity $[\mathrm{dB}]$................................................................45

Abbildung 31: Gemittelte Ischämiefläche $\left[\mathrm{mm}^{2}\right]$ vs. mean defect $[\mathrm{dB}]$......................................................................46

Abbildung 32: Gemittelte Ischämiefläche $\left[\mathrm{mm}^{2}\right]$ vs. center point thickness $[\mu \mathrm{m}]$ ].....................................................4

Abbildung 33: Gemittelte Ischämiefläche $\left[\mathrm{mm}^{2}\right]$ vs. center subfield thickness $[\mu \mathrm{m}]$................................................48

Abbildung 34: Gemittelte Ischämiefläche $\left[\mathrm{mm}^{2}\right]$ vs. total macular volume $\left[\mathrm{mm}^{3}\right]$.................................................49

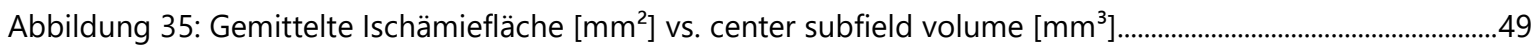




\section{Tabellenverzeichnis}

Tabelle 1: Stadien der diabetischen Retinopathie ............................................................................................................... 3

Tabelle 2: Kriterien des klinisch signifikaten Makulaödems .............................................................................................

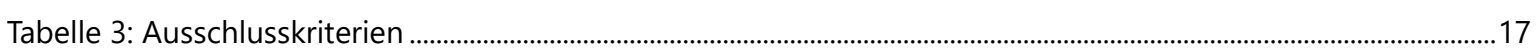

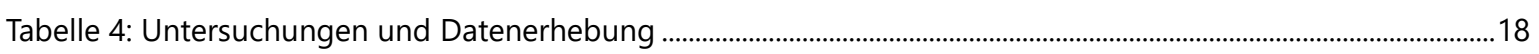

Tabelle 5: Demographie und Patienten-Charakteristika.............................................................................................................22

Tabelle 6: Diabetesbezogene Daten .....................................................................................................................................28

Tabelle 7: Demographie und Patienten-Charakteristika, Schwellenwert-Analyse .........................................................22

Tabelle 8: Diabetesbezogene Daten, Quartilanalyse ...........................................................................................................29

Tabelle 9: Sphärisches Äquivalent, Quartil- und Schwellenwert-Analyse..........................................................................32

Tabelle 10: Visus- und Kontrastvisusbestimmung, Schwellenwert-Analyse .......................................................................33

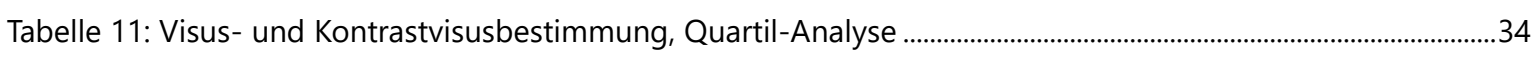

Tabelle 12: Gesichtsfeldmessung, Schwellenwert-Analyse...............................................................................................35

Tabelle 13: Gesichtsfeldmessung, Quartil-Analyse ..........................................................................................................................35

Tabelle 14: OCT-Parameter im Vergleich, Schwellenwert-Analyse ........................................................................................3

Tabelle 15: OCT-Parameter im Vergleich, Quartil-Analyse ....................................................................................................38

Tabelle 16: Übersicht Korrelationswerte Ischämiefläche vs. demografische Daten ......................................................42

Tabelle 17: Übersicht Korrelationswerte Ischämiefläche vs. Visus.........................................................................................4

Tabelle 18: Übersicht Korrelationswerte Ischämiefläche vs. perimetrische Daten ...........................................................46

Tabelle 19: Übersicht Korrelationswerte Ischämiefläche vs. OCT-Ergebnisse..................................................................50

Tabelle 20: Vergleich Normwerte vs. Studienergebnisse............................................................................................................53

Tabelle 21: Übersicht retinale Schichtdicken bei Patienten mit Diabetes mellitus ........................................................54 


\section{Abkürzungsverzeichnis}

$\begin{array}{ll}\text { BM } & \text { Basalmembran } \\ \text { CSFV } & \text { central subfield volume } \\ \text { CSFT } & \text { central subfield thickness } \\ \text { CPT } & \text { central point thickness } \\ \text { DM } & \text { Diabetes mellitus } \\ \text { dpt } & \text { Dioptrien } \\ \text { DR } & \text { diabetische Retinopathie } \\ \text { ETDRS } & \text { Early Treatment Diabetic Retinopathy Study } \\ \text { FA } & \text { Fluoreszeinangiografie } \\ \text { FAZ } & \text { foveale avaskuläre Zone } \\ \text { GCL } & \text { ganglion cell layer } \\ \text { ICT } & \text { intense conventional therapy } \\ \text { IVOM } & \text { intravitreale operative Medikamenteneingabe } \\ \text { LA } & \text { linkes Auge } \\ \text { MD } & \text { mean defect } \\ \text { MS } & \text { mean sensitivity } \\ \text { N } & \text { Größe der Grundgesamtheit } \\ \text { NPDR } & \text { nicht-proliferative diabetische Retinopathie } \\ \text { OCT } & \text { optische Kohärenztomografie } \\ \text { OCT-A } & \text { optische Kohärenztomografie-Angiografie } \\ \text { PDR } & \text { proliferative diabetische Retinopathie } \\ \text { RA } & \text { rechtes Auge } \\ \text { SÄ } & \text { sphärisches Äquivalent } \\ \text { SD } & \text { Standardabweichung } \\ \text { TMV } & \text { total macular volume } \\ \text { VAS } & \text { Visual Acuity Score } \\ \text { VEGF } & \text { vascular endothelial growth factor } \\ & \end{array}$




\section{Einleitung}

Die diabetische Retinopathie betrifft global mehr als ein Drittel der an Diabetes mellitus erkrankten Menschen und gehört somit zu den häufigsten Augenerkrankungen weltweit (Yau et al. 2012). Folge dieser Erkrankung kann unter anderem ein progredienter Visusverlust sein, welcher bis zur Erblindung führen kann. Hieraus resultieren sowohl erhebliche Einschränkungen des Patienten im Alltag bis hin zur Erwerbsminderung oder Erwerbsunfähigkeit. Die intensive Aufklärung der Patienten und die Prävention der diabetischen Retinopathie stellt deshalb ein wichtiges Ziel innerhalb der medizinischen Versorgung dar. Eine Voraussetzung hierfür ist das Verständnis der pathophysiologischen Zusammenhänge sowie ihrer funktionellen und morphologischen Korrelate im diabetischen Auge.

Die vorliegende klinische Studie untersucht sowohl funktionelle als auch morphologische Parameter bei Typ-1 und Typ-2-Diabetikern. Ziel ist es, den Zusammenhang zwischen den krankheitsbedingten morphologischen Veränderungen und den funktionellen Testergebnissen zu überprüfen. Im Detail soll geklärt werden, ob und ab welchem Zeitpunkt sich eine zentrale Durchblutungsstörung (makuläre Ischämie) auf die Sehfunktion auswirkt und in welchem Maß die Schwere der Ischämie mit der Einschränkung der Sehfunktion assoziiert ist. Den Kern der Studie bildet deshalb die Beurteilung des Augenhintergrundes mithilfe der Bildgebungsmethoden Fundusfotografie und Fluoreszeinangiografie. Zur Beurteilung der Sehfunktion wurden verschiedene Tests durchgeführt; unter anderem die Untersuchung der Sehkraft und des Gesichtsfeldes.

\subsection{Die diabetische Retinopathie}

\subsubsection{Terminologie und Klassifikation}

Als diabetische Retinopathie bezeichnet man die durch einen Diabetes mellitus verursachte Mikroangiopathie der Netzhaut. Klinisch wird sie in eine nicht-proliferative diabetische Retinopathie (NPDR) und eine proliferative diabetische Retinopathie (PDR) unterteilt. Diese Differenzierung dient zur Spezifizierung des Schweregrads der Erkrankung und hat therapeutische Konsequenzen. Auch die Lokalisation der Schädigung ist von Bedeutung, weshalb immer Stellung dazu genommen werden muss, ob auch das Netzhautzentrum (Makula) betroffen ist, was dann als diabetische Makulopathie bezeichnet wird. Bei der Beurteilung der Makula wird zwischen einer reinen 
Durchblutungsstörung (makuläre Ischämie) und einer Schrankenstörung (Makulaödem) unterschieden.

Im Mittelpunkt der vorliegenden Arbeit stehen diabetische Veränderungen der Makula und der Fovea centralis, dem Punkt des schärfsten Sehens. An dieser anatomischen Stelle gibt es keine Gefäße, das Gebiet wird als foveale avaskuläre Zone (FAZ) bezeichnet. Die durchschnittliche Größe der FAZ beträgt etwa 0,3-0,4 mm² (Dubis et al. 2012; Guo et al. 2017). Ein Normalbefund der FAZ wird in Abbildung 1 gezeigt.

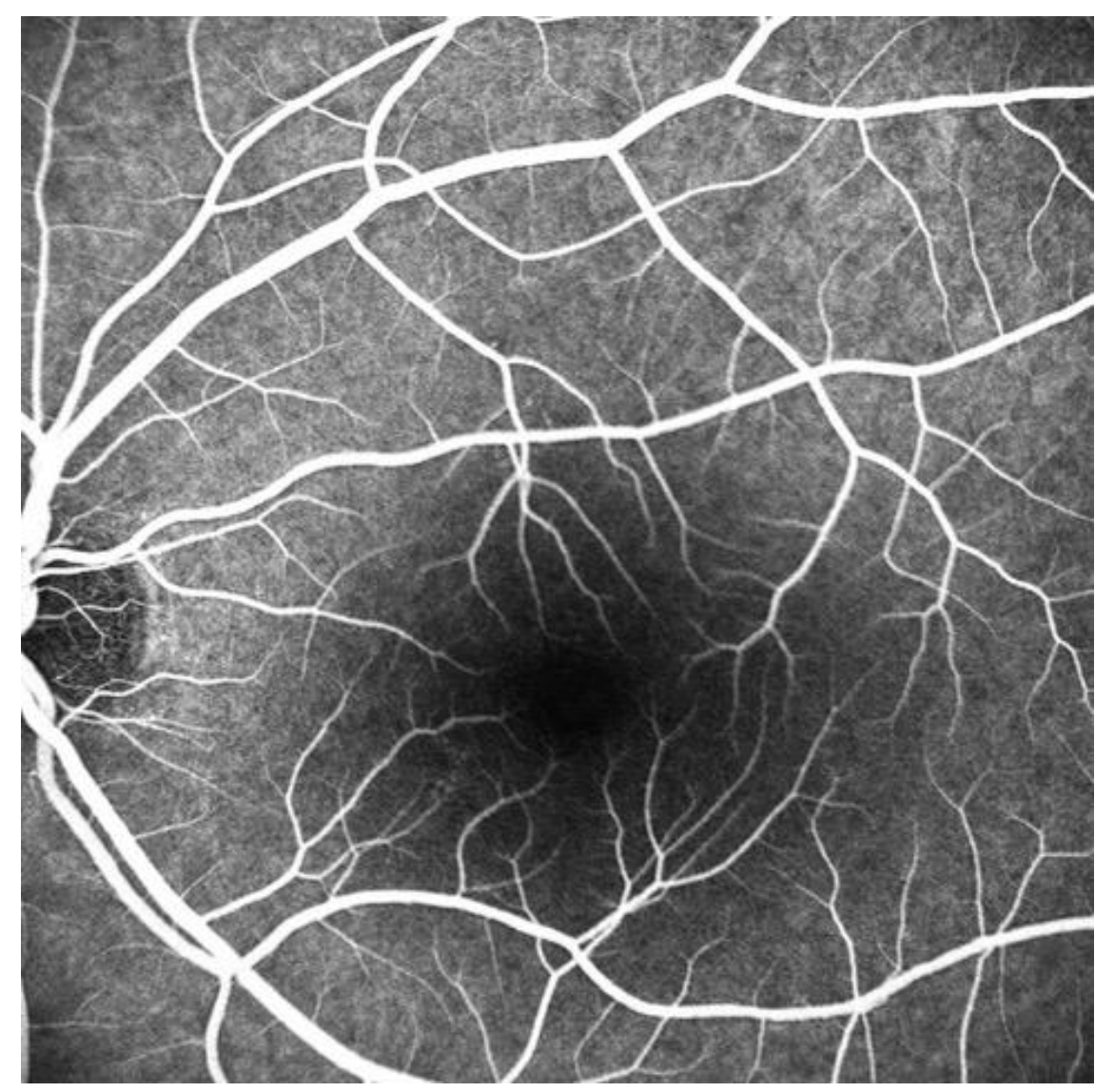

Abbildung 1: Foveale avaskuläre Zone in der Fluoreszeinangiografie, Normalbefund (mit freundlicher Genehmigung der Augenklinik der UMG)

Die NPDR ist gekennzeichnet durch Mikroaneurysmen, Punkt- und Fleckblutungen, Cotton-woolHerde, harte Exsudate und, bei zentralem Befall, einem Makulaödem (siehe Abbildung 2). Mikroaneurysmen entstehen durch eine Wandschwäche der retinalen Kapillaren. Risse in diesen Mikroaneurysmen können zu Blutungen in den oberflächlichen Netzhautschichten führen. Eine protrahierte Schrankenstörung führt zu einer Ödembildung, die bei zentraler Beteiligung zu erheblichen Einbußen der Sehschärfe führen kann. Neben der Diffusion von Albumin und Blut aus 
den mikroangiopathisch veränderten Kapillaren, finden sich außerdem Lipide im Extrazellularraum, die als harte Exsudate bezeichnet werden. Im Verlauf der Erkrankung kommt es regelhaft zu Gefäßokklusionen mit ischämischen Arealen im nachgeschalteten Stromgebiet. Durch ischämische Reaktionen im Nervenfaserverlauf schwellen die retinalen Axone an, es kommt zu einem Axonplasmastau, der funduskopisch als weiß-gelbliche Verschattungen detektiert werden kann (sog. Cotton-wool-Herde).

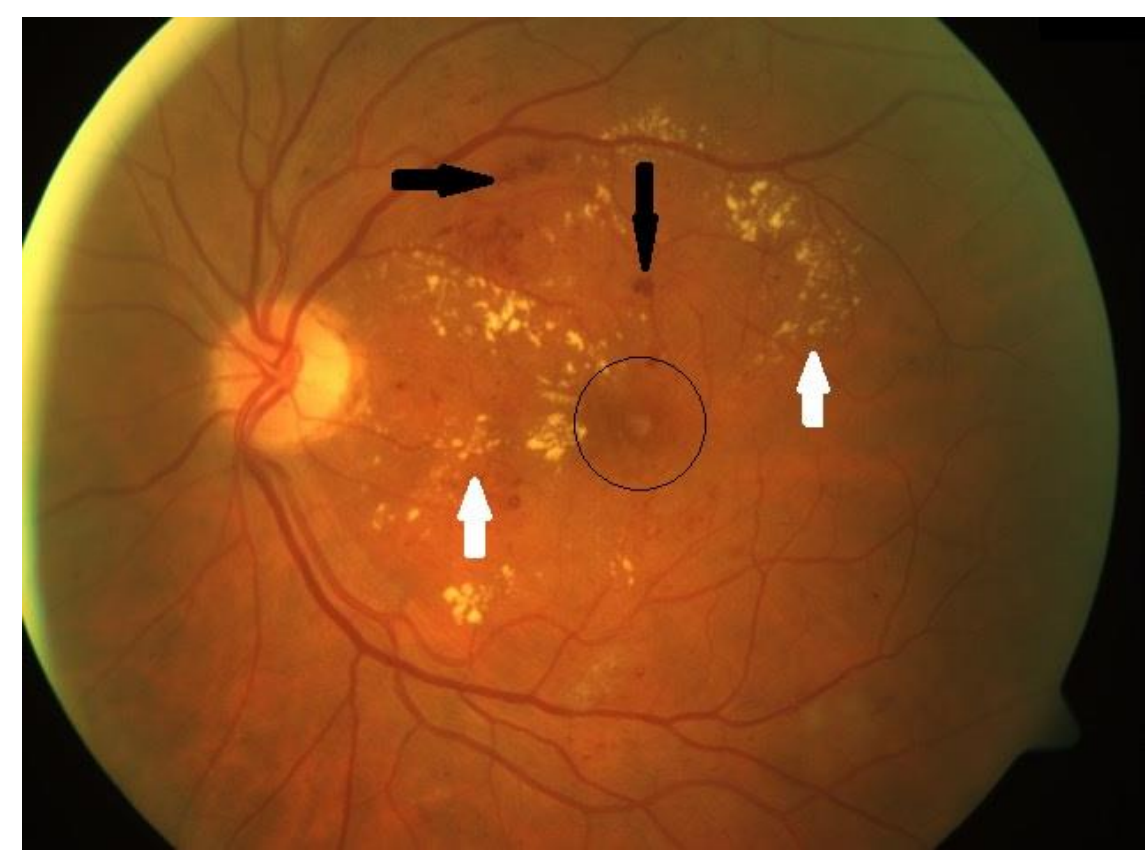

Abbildung 2: Diabetische Makulopathie mit Einblutungen (schwarze Pfeile), harten Exsudaten (weiße Pfeile) und Makulaödem (schwarzer Kreis), Fundusfotografie (mit freundlicher Genehmigung der Augenklinik der UMG)

Die NPDR lässt sich klinisch in drei Stadien einteilen (siehe Tabelle 1), die oben genannten mikrovaskulären Veränderungen helfen bei der Unterteilung.

Tabelle 1: Stadien der diabetischen Retinopathie (Bundesärztekammer (BÄK) et al. 2015)

\begin{tabular}{|l|l|}
\hline Form & \multicolumn{1}{|c|}{ Klinisches Bild } \\
\hline Milde NPDR & Nur einzelne Mikroaneurysmen \\
\hline Mäßige NPDR & $\begin{array}{l}\text { Mikroaneurysmen, intraretinale Blutungen, maximal ein Quadrant mit } \\
\text { perlschnurartigen Venen }\end{array}$ \\
\hline Schwere NPDR & $\begin{array}{l}\text { Mehr als 20 Mikroaneurysmen und/oder intraretinale Blutungen in jedem } \\
\text { der vier Quadraten und/oder mindestens eine perlschnurartige Vene in } \\
\text { mindestens zwei Quadranten und/oder mindestens eine intraretinale } \\
\text { mikrovaskuläre Anomalie (IRMA) in mindestens einem Quadranten }\end{array}$ \\
\hline PDR & Neovaskularisationen \\
\hline
\end{tabular}


Die Einteilung des Makulaödems wurde in der Early Treatment for Diabetic Retinopathy Study (ETDRS) vorgenommen und hat bis heute Gültigkeit (Bundesärztekammer (BÄK) et al. 2015; Mathew et al. 2015). Kriterien, die für die Definition eines klinisch signifikanten Makulaödems im Rahmen der ETDRS-Studie festgelegt wurden, sind in Tabelle 2 aufgeführt.

Tabelle 2: Kriterien des klinisch signifikaten Makulaödems (Bundesärztekammer (BÄK) et al. 2015)

Signifikantes Makulaödem (Wenn mindestens ein Kriterium erfüllt ist)

Netzhautödem reicht bis in die Fovea centralis (foveale Beteiligung)

Netzhautödem reicht bis zu $500 \mu \mathrm{m}$ vom Zentrum, evtl. mit harten Exsudaten

Netzhautödem reicht bis $1500 \mu \mathrm{m}$ vom Zentrum und Fläche $\geq 1$ Papillenfläche

Die PDR weist additiv zu den Veränderungen der NPDR charakteristisch Neovaskularisationen auf (sog. Proliferationen) (Witmer et. al. 2003). Diese neu entstandenen, pathologischen Gefäße sind häufig besonders fragil, weshalb sie typischerweise mit einer Schrankenstörung und Blutungen einhergehen. Die Proliferationen wachsen zunächst in der Netzhaut und werden als intraretinale mikrovaskuläre Anomalien (IRMA) bezeichnet. Gerade bei noch anliegendem Glaskörper tendieren sie aber dazu vertikal auszusprießen und in das Glaskörpergerüst zu proliferieren. Durch die Fibrosierungsneigung der Neovaskularisationen einerseits und die physiologische Degeneration des Glaskörpers andererseits kann es zu Traktionen an den Proliferationen mit spontaner Blutung kommen. Unbehandelt besteht auch im vorderen Augenabschnitt ein neovaskulärer Reiz, der dann zu Gefäßproliferationen auf der Iris bis hin zu Störungen des Kammerwasserabflusses und fulminantem Augeninnendruckanstieg führen kann (rubeotisches Sekundärglaukom). Auch die PDR lässt sich in verschiedene Stadien einteilen, wobei die klinische Relevanz geringer ist als bei der NPDR. Die diabetische Retinopathie kann anhand der ETDRS-Skala unterteilt werden (siehe Abbildung 3). Diese Skala suggeriert aufgrund der angegebenen Zahlen eine Genauigkeit, die im klinischen Alltag allerdings nicht gegeben ist. Deshalb hat sie sich in Zentraleuropa bisher nicht durchgesetzt. 


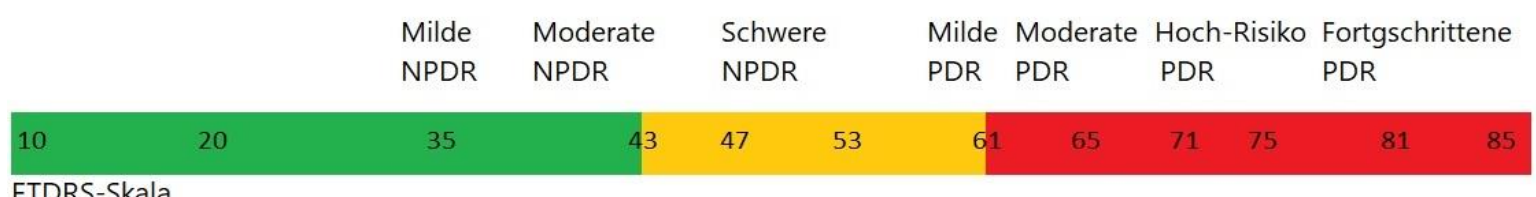

Abbildung 3: Schweregradeinteilung der diabetischen Retinopathie anhand der ETDRS-Skala. Dabei ist die ETDRS-Skala das in den US-amerikanischen Studien präferierte System, während in Europa die obere, beschreibende Einteilung verwendet wird (siehe Tabelle 1 und Tabelle 2), Abbildung erstellt nach (Davis et al. 1998)

Bei der Beurteilung der Beteiligung der Netzhautmitte wird nach Ödembildung und retinaler Ischämie gesucht. Während ein Ödem regelhaft mit einer Sehverschlechterung einhergeht (Ahmadpour-Baghdadabad et al. 2013; Ramsey und Arden 2015), kann der Visus bei einer ischämischen Makulopathie zunächst noch wenig verändert sein.

\subsubsection{Epidemiologie}

Die diabetische Retinopathie ist die häufigste Erblindungsursache von Patienten im Alter von 20 bis 74 Jahren weltweit (Lee et al. 2015). Bezieht man auch Menschen außerhalb des erwerbsfähigen Alters mit ein, führt nur die altersabhängige Makuladegenration (AMD) noch häufiger zu Blindheit (NVL Diabetische Retinopathie 2016). Der Sehverlust bei Diabetikern (Typ-1 und Typ-2) wird am häufigsten durch das diabetische Makulaödem verursacht (Lightman und Towler 2003; Acan et al. 2018). Das diabetische Makulaödem ist zugleich die häufigste vaskuläre Ursache eines Visusverlusts in den westlichen Industrieländern (Ding und Wong 2012). Zudem wurde nachgewiesen, dass die diabetische Retinopathie sowie das Makulaödem mit der Entwicklung von weiteren diabetesassoziierten Folgeschäden, wie zum Beispiel die diabetische Nephropathie und Neuropathie, assoziiert sind (Lee et al. 2014; Mottl et al. 2014; Hammes et al. 2015; Zhang et al. 2018). Das hat für die Kommunikation und den Informationsaustausch zwischen den Augenärzten und den Diabetologen eine entscheidende Bedeutung.

Von weltweit etwa 285 Millionen Patienten mit Diabetes mellitus ist ungefähr jeder dritte Patient von einer diabetischen Retinopathie betroffen. Von dieser Gruppe ist wiederum ein Drittel von einem unmittelbaren Sehverlust bedroht (Yau et al. 2012). Die Prävalenz der diabetischen Retinopathie liegt bei Typ-1-Diabetikern bei ca. 24-27\% bei Typ-2-Diabetikern bei ca. 9-16\% (NVL Diabetische Retinopathie 2016; Ponto et al. 2016). In Studien konnte zudem nachgewiesen werden, dass die Prävalenz der Retinopathie mit zunehmender Erkrankungsdauer des Diabetes mellitus 
ansteigt. Nach 40 Krankheitsjahren weisen ungefähr $84 \%$ der Typ-1 und bis zu 63\% der Typ-2 Diabetiker Zeichen einer diabetischen Retinopathie auf (Hammes et al. 2011; Voigt et al. 2018).

\subsubsection{Pathophysiologie}

Die diabetische Retinopathie entsteht durch die langanhaltende Schädigung der Gefäßendothelien (Avogaro et al. 2011). Die Ablagerung von glykierten Makromolekülen in den Gefäßwänden führt zu einer Mikroangiopathie der Kapillaren durch Destabilisierung ihres Grundgerüstes (Madonna et al. 2017). Aus der anhaltenden Entzündung resultiert der Untergang von Perizyten und Endothelzellen. Folge der vaskulären Dysfunktion ist der Ausfall der Blut-Retina-Schranke und Okklusion der Kapillaren, die zur Minderperfusion der Retina führen. Diese Minderperfusion betrifft hierbei alle retinalen Schichten und Zelltypen (Cohen und Gardner 2016). Zeitgleich mit der Entwicklung einer diabetischen Retinopathie finden sich nicht selten bestimmte Veränderungen des Blutbilds, unter anderem eine gesteigerte Erythrozytenaggregation, eine ineffektive Fibrinolyse, eine gesteigerte Serumkonzentrationen von Wachstumshormonen, wie dem vascular endothelial growth factor (VEGF), sowie Zeichen von lokaler und systemischer Entzündung. Der Zusammenhang zwischen dem gemeinsamen Auftreten der Blutbildveränderungen und einer diabetischen Retinopathie ist zwar sehr naheliegend, allerdings konnte bisher keine direkte Korrelation nachgewiesen werden (Antonetti et al. 2012). VEGF spielt neben den Entzündungsmediatoren eine besondere Rolle in der Veränderung der Blut-Retina-Schranke (Chowdhury et al. 2002). Die durch Hypoxie und Ischämie gesteigerte Produktion von VEGF führt zur Veränderung von Proteinen durch Phosphorylierung, welche für die Integrität von Zell-Zell-Kontakten eine Rolle spielen. Diese Veränderung manifestiert sich klinisch als Makulaödem (Antonetti et al. 1999; Simó und Hernández 2008).

\subsubsection{Risikofaktoren}

\subsubsection{Blutzucker}

In der Wisconsin Epidemiologic Study of Diabetic Retinopathy konnten bereits 1984 wichtige Risikofaktoren wie u.a. die Erkrankungsdauer, männliches Geschlecht, hohe Langzeitzuckerwerte $\left(\mathrm{HbA}_{1 c}\right)$ und ein hoher diastolischer Blutdruckwert nachgewiesen werden (Klein et al. 1984). Diese Ergebnisse konnten auch in jüngeren Studien und Metaanalysen bestätigt werden. Ein langanhaltend zu hoher Blutzucker, gemeint sind hier sowohl der Kapillarglukosewert, als auch ein $\mathrm{HbA}_{1 c}>8 \%$, stellt einen der wichtigsten Risikofaktoren für die Entwicklung einer diabetischen 
Retinopathie dar (Hemmingsen et al. 2011; Yau et al. 2012). Dies gilt sowohl für Patienten mit einem Typ-1 (Hermann et al. 2014), als auch mit einem Typ-2 Diabetes mellitus (Hammes et al. 2015). Bezogen auf die Progression einer bereits vorhandenen diabetischen Retinopathie konnte gezeigt werden, dass diese beim Typ-1 Diabetes vom $\mathrm{HbA}_{1 c}$ abhängt (Diabetes Control and Complications Trial Research Group et al. 1993; Kawasaki et al. 2011; Broe et al. 2014; Group 2015). Beim Typ-2 Diabetes liegen sowohl Studien vor, die ebenso einen Zusammenhang nachweisen konnten (UK Prospective Diabetes Study (UKPDS) Group 1998; ACCORD Study Group et al. 2010; Ferris und Nathan 2016), als auch Studien, in denen kein Zusammenhang zwischen den Langzeitzuckerwerten und dem Fortschreiten einer diabetischen Retinopathie festgestellt werden konnte (Beulens et al. 2009; Duckworth et al. 2009). Es konnte zudem gut untersucht werden, dass die Einleitung einer intensivierten Therapie vor allem bei bereits lang andauernder Therapie oder schlecht eingestellten Patienten mit Diabetes mellitus mit einer vorbestehenden diabetischen Retinopathie zu einem so genannten early worsening führen kann, also einer rapiden Zunahme der diabetischen Retinopathie (Hooymans et al. 1982; Feldman-Billard et al. 2018; Bain et al. 2019). Die Einstellung des Langzeitzuckerwerts innerhalb des individuellen Zielbereiches, primär mittels eines intensivierten konservativen Schemas, stellt trotzdem weiterhin einen zentralen Gesichtspunkt in der Therapie einer diabetischen Retinopathie dar, da sie die Krankheitsentstehung verhindern können (Bundesärztekammer (BÄK) et al. 2015). Es ist nicht bekannt, inwieweit die Anpassungsgeschwindigkeit oder die Verringerung der Amplitude des $\mathrm{HbA}_{1 c}$ einen Einfluss auf die Reduzierung der Auftretenswahrscheinlichkeit eines early worsening haben.

\subsubsection{Bluthochdruck und Nephropathie}

Ebenfalls von maßgeblicher Bedeutung ist das häufig gemeinsame Auftreten von erhöhten Blutzuckerwerten und arterieller Hypertension, da die Schädigung der retinalen Blutgefäße durch einen erhöhten Gefäßinnendruck weiter vorangetrieben werden kann (Patel et al. 1992; Raum et al. 2015). Die Behandlung eines koexistierenden arteriellen Hypertonus ist von entscheidender therapeutischer Bedeutung. Er ist zu einem großen Teil mitverantwortlich für die Entstehung oder das Fortschreiten einer diabetischen Retinopathie (Saif et al. 2014). In der U.K. Prospective Diabetes Study (UKPDS) konnte nachgewiesen werden, dass eine enge Kontrolle der Blutdruckwerte $(<150 / 85 \mathrm{mmHg}$ ) über einen Zeitraum von etwa acht Jahren den Progress einer Retinopathie um etwa 34\% reduzieren konnte (Stratton et al. 2001). Auch die Auftrittswahrscheinlichkeit einer diabetischen Retinopathie bei an Diabetes erkrankten Patienten lässt sich durch eine konsequente Blutdruckkontrolle um bis zu vier bis fünf Jahre senken (Do et al. 2015). Der gleiche Pathomechanismus ist außerdem mitverantwortlich für die Entstehung einer diabetischen 
Nephropathie, die in einer engen Beziehung mit der diabetischen Retinopathie steht. Liegt bei einem Patienten eine Beteiligung der Niere vor, sollte die augenfachärztliche Kontrolle von besonderem Stellenwert sein, da sowohl der Nachweis oder die Progression der diabetischen Retinopathie bei gleichzeitiger diabetische Nierenschädigung häufiger zu beobachten ist (NVL Diabetische Retinopathie 2016). Als Parameter korreliert die Mikro- als auch Makroalbuminurie mit der Entwicklung einer diabetischen Retinopathie (Hammes et al. 2015).

\subsubsection{Blutfette}

Betrachtet man die Rolle von erhöhten Blutfettwerten als Risikofaktor für eine diabetische Retinopathie, bemerkt man eine deutliche Inkonsistenz der Ergebnisse im Gegensatz zu anderen Risikofaktoren. Es wurden sowohl Korrelationen für erhöhte Triglyceridwerte (Hadjadj et al. 2004; Rema et al. 2006), als auch für erhöhte low-density-lipoprotein (LDL)-Werte beschrieben (Klein et al. 2002b; Popescu und Moţa 2009). Die vermehrte Bildung von harten Exsudaten konnte sowohl bei gesteigerten Blutwerten für Triglyceride (Klein et al. 2002a), als auch für Gesamtcholesterin und LDL (Chew et al. 1996; Uçgun et al. 2007; Sachdev und Sahni 2010) nachgewiesen werden. Eine neuere Untersuchung beschreibt eine Korrelation zwischen Entwicklung der diabetischen Retinopathie und erhöhten HDL-Werten bei asiatischen Patienten. Die widersprüchlichen Ergebnisse sind möglicherweise auf die unterschiedlichen Bevölkerungsgruppen zurückzuführen und nicht unbedingt seriöse Risikofaktoren.

\subsubsection{Weitere Faktoren}

Auch hormonelle Schwankungen, wie sie in der Schwangerschaft und Pubertät typisch sind, stellen einen Risikofaktor für die Entwicklung und den Progress der diabetischen Augenkomplikationen dar (Mallika et al. 2010). Deshalb sind diese Lebensabschnitte hochvulnerable Phasen für die Progression einer diabetischen Retinopathie. Für den Diabetes Typ-1 ist zudem nachgewiesen worden, dass Zigarettenrauchen das Auftreten einer diabetischen Retinopathie begünstigt (Praxisleitlinie der Deutschen Diabetes Gesellschaft 2016). Neben metabolischen Risikofaktoren ist außerdem bekannt, dass bestimmte ethnische Gruppen sowohl von Diabetes mellitus als auch von den resultierenden Folgeschäden, wie der diabetischen Retinopathie, häufiger betroffen sind (Yau et al. 2012; Islam et al. 2016). 


\subsubsection{Diagnostik}

Zur Untersuchung einer diabetischen Retinopathie werden verschiedene Verfahren angewendet. Zur standardmäßig durchgeführten Diagnostik gehören die Testung von Refraktion, Visus und Gesichtsfeld sowie die Schichtdickenmessung der Retina und die Fundusfotografie bzw. Fluoreszeinangiografie (FA).

\subsubsection{Refraktion und sphärisches Äquivalent}

Mithilfe der Refraktion und des sphärischen Äquivalents (SÄ) lässt sich der reelle Gesamtbrechwert des optischen Systems abbilden. Der Einfluss von Vergrößerungs- bzw. Verkleinerungseffekten sowie der Grad einer Hornhautverkrümmung (Astigmatismus) können so untersucht werden.

Das sphärische Äquivalent sich zusammen aus der Addition der Stärken von Sphäre und Zylinder und wird in dpt (oder D) angegeben (siehe Abbildung 4).

$$
\pm \text { Sphäre } \pm\left(\frac{1}{2}\right) \times \text { Zylinder }=\text { sphärisches Äquivalent }
$$

\section{Abbildung 4: Berechnung des sphärischen Äquivalents}

\subsubsection{Visus und Kontrastvisus}

Bei der Sehschärfenprüfung wird mittels projizierten Sehzeichen in abnehmender Größe die Sehschärfe des Probanden geprüft. Die Untersuchung ist leicht reproduzierbar und kann im Verlauf gut kontrolliert werden. Zusätzlich zum Visus besteht die Möglichkeit der Überprüfung des Kontrastsehens. Dabei wird gemessen, wie gut der Patient Helligkeitsunterschiede zwischen den inm dargebotenen Sehzeichen unterscheiden kann. Der verwendete Logarithmus in Bezug auf die Größenabstufung der dargebotenen Sehzeichen ist für die Sehschärfenprüfung durch die DINNormen festgelegt. Sowohl für den Visus, als auch für den Kontrastvisus liegt die Abstufung bei $\sqrt[10]{10}$ (Lachenmayr et al. 2006). Der Visus ergibt sich aus der Formel Visus $=\frac{\text { Prüfentfernung }}{\text { Normalentfernung }}$. Je größer der Nenner, desto schlechter die Sehschärfe. Als Normalwert gilt ein Dezimalvisus von 1,0. Da die traditionellen Sehtafeln nicht logarithmiert dargestellt sind, muss für Studienuntersuchungen eine gesonderte Tafel verwendet werden. Die Sehschärfe wird hierbei in gelesenen Buchstaben oder einem logarithmierten Wert angegeben (logMAR), der dann wieder in einen Dezimalvisus zurückgerechnet werden kann (siehe auch 2.3.3). 


\subsubsection{Perimetrie}

Im Rahmen der Bestimmung des Gesichtsfeldes werden dem Patienten Lichtpunkte in unterschiedlichen Abständen zum zentralen Gesichtsfeld dargeboten, die in ihrer Helligkeit so lange gesteigert werden, bis sie vom Patienten wahrgenommen werden. Auf diese Weise wird nach und nach eine Karte mit den Außengrenzen des zentralen Gesichtsfeldes des Patienten angefertigt. Gemessen werden die Schwellenwerte in Bezug auf die Empfindlichkeit in der Einheit Dezibel (dB). Dabei gilt, je höher der angegebene Wert, desto besser ist die Lichtempfindlichkeit für den Prüfort. Zusätzlich werden dem Patienten durch das Perimeter automatisiert in zufälligen Abständen „Fangfragen" präsentiert. Es handelte sich um optische Reize, die sich entweder außerhalb des Gesichtsfeldes befinden oder fehlen und somit vom Patienten nicht wahrgenommen werden können. Der Anteil der bejahten Fangfragen dient als Merkmal zur Qualitätsüberprüfung der perimetrischen Untersuchung. Die Qualität der Untersuchung verbessert sich, je weniger Fangfragen bejaht wurden. Ein Nachteil der zusätzlichen Fangfragen kann die Verlängerung der Untersuchungszeit sein, die dazu führen kann, dass der Patient unkonzentrierter arbeitet und vor allem zum Ende der Datenerhebung hin mehr Fehler macht, was die Qualität der Untersuchung vermindern kann (Katz et al. 1991).

\subsubsection{Optische Kohärenztomografie (OCT)}

Die OCT-Untersuchung dient dazu mittels eines spaltförmigen Laserstrahls einen Querschnitt der Netzhaut anzufertigen. Der Strahl wird zu diesem Zwecke auf die Netzhaut projiziert und das reflektierte Licht analysiert. Das entstehende Bild ermöglicht es dem Untersucher die unterschiedlichen Schichten der Netzhaut zu differenzieren, und Veränderungen einer bestimmten Schicht zuzuordnen (siehe Abbildung 5). Die unterschiedliche Reflexion des Lichtes hängt mit dem Aufbau und der Zusammensetzung der einzelnen Retinaschichten zusammen. An den Grenzflächen verändert sich die Brechung des Lichts, was in der Rekonstruktion als Schichtaufbau erkennbar ist. 


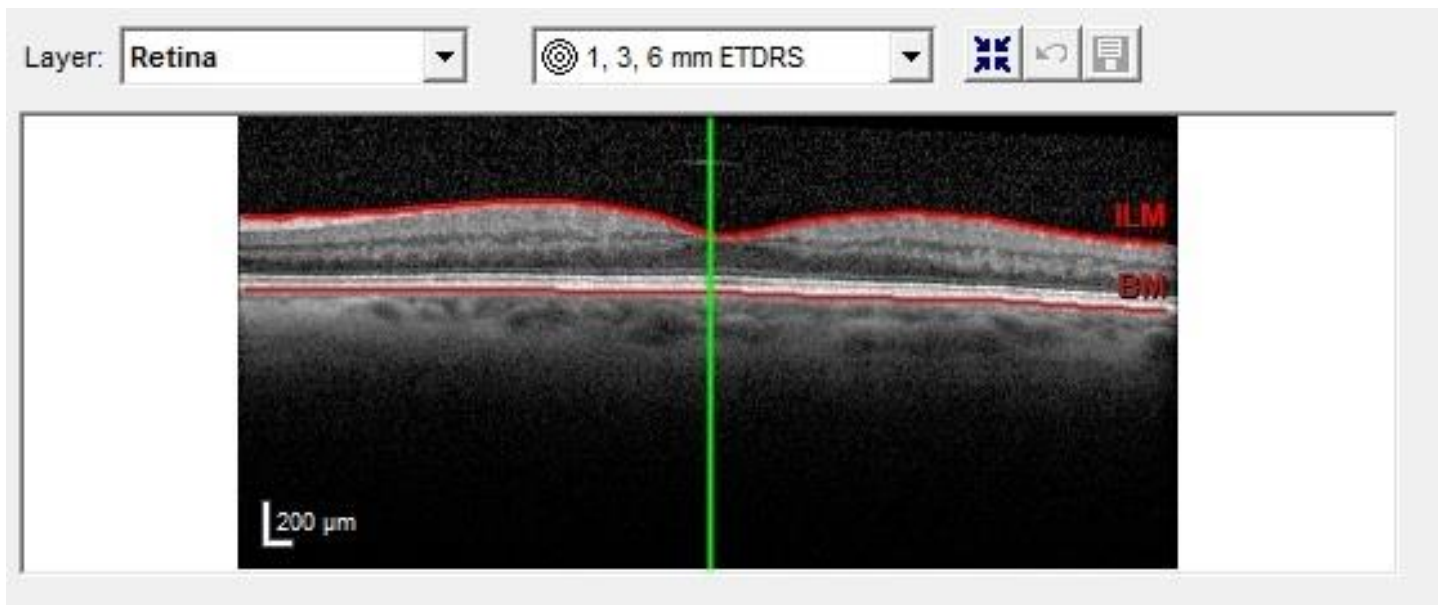

Abbildung 5: Darstellung der Fovea (Höhe des grünen Balkens) und Retina (Bereich zwischen den roten Markierungen) mittels OCT im Querschnitt (mit freundlicher Genehmigung der Augenklinik der UMG)

\subsubsection{Fundusfotografie und Fluoreszeinangiografie}

Bei der Fundusfotografie werden mithilfe einer Kamera Bilder des Augenhintergrunds aufgenommen, die Aufschluss über morphologische Veränderungen der Netzhaut bieten (Beispiel siehe Abbildung 6). Die Fluoreszeinangiografie dient als Erweiterung der Fundusfotografie, bei der dem Patienten ein Farbstoff (z.B. 5 ml Fluoreszein) venös injiziert wird. Durch die Applikation in den Blutkreislauf lassen sich besonders vaskuläre Prozesse in der Netzhaut gut beurteilen (Beispiel siehe Abbildung 7). Dazu zählen unter anderem ischämische, aber auch proliferative Prozesse.

Durch die Filterung des einfallenden Lichts gelangt nur blaues Licht mit einer Wellenlänge von etwa $480 \mathrm{~nm}$ bis zur Netzhaut und regt dort den applizierten Farbstoff in den Gefäßen zur Fluoreszenz an. Die entstehende grün-gelbe Fluoreszenz mit einer Länge von ca. $525 \mathrm{~nm}$ wird reflektiert und das Bild traditionell auf einem schwarz-weiß-Film der Funduskamera abgebildet. Ein Filter für grüngelbes Licht zwischen Patientenauge und Kamera sorgt dafür, dass nur das durch die Fluoreszenz hervorgerufene Licht auf den Film gelangt (Sachsenweger u. a. 2002). Moderne Geräte verwenden im Gegensatz zum herkömmlichen Film heutzutage zunehmend digitale Systeme in der Bildverarbeitung und Bildspeicherung. Sie erleichtern vor allem den Zugriff auf die entstandenen Bilder und ermöglichen deren Bearbeitung zur besseren Darstellung der aufgezeigten Pathologien. 


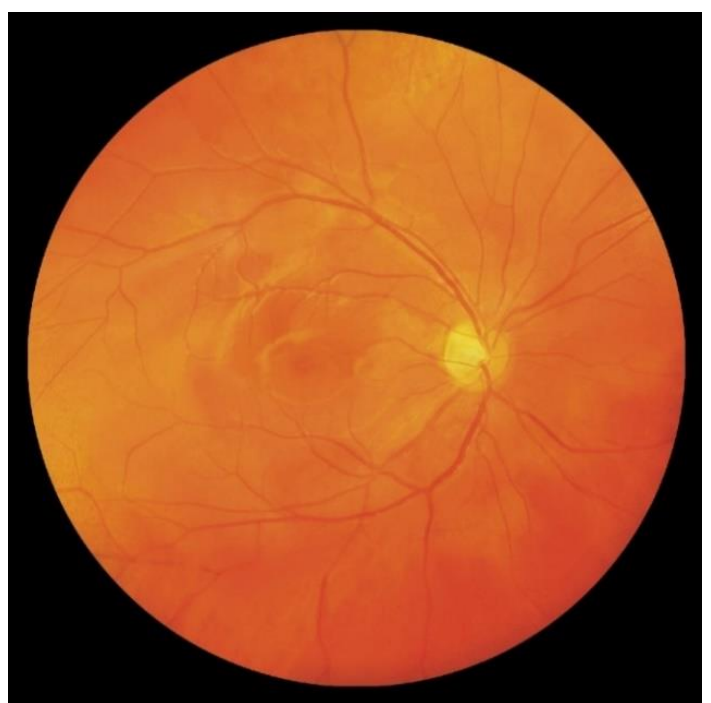

Abbildung 6: $50^{\circ}$ Fundusaufnahme einer gesunden Netzhaut (mit freundlicher Genehmigung der Augenklinik der UMG)

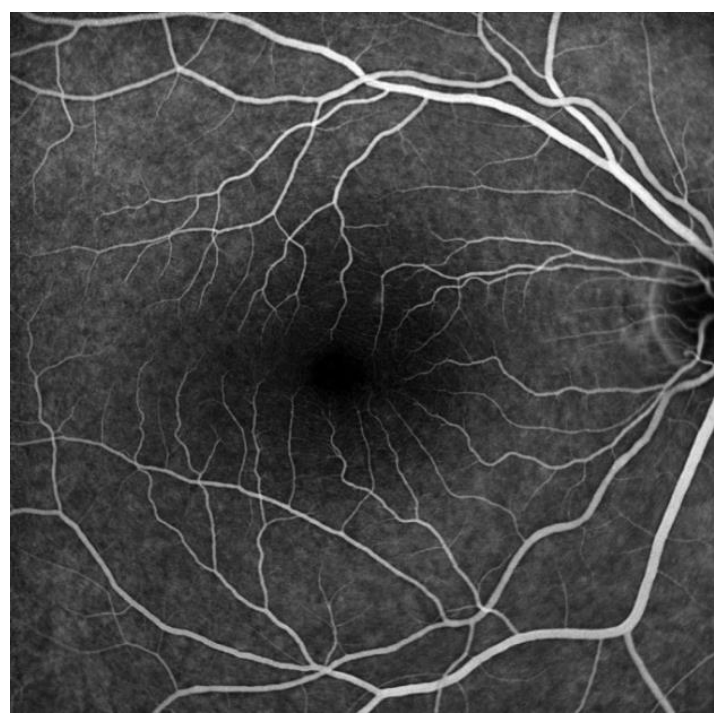

Abbildung 7: Mittlere Phase einer Fluoreszeinangiografie einer gesunden Netzhaut, $30^{\circ}$-Ausschnitt (mit freundlicher Genehmigung der Augenklinik der UMG)

\subsubsection{Behandlung}

Bei der Behandlung der diabetischen Retinopathie ist zunächst die Behandlung bzw. Vermeidung von Risikofaktoren entscheidend. So kann sowohl die Auftretenswahrscheinlichkeit reduziert als auch ein Krankheitsprogress verlangsamt oder sogar gestoppt werden.

Basis der augenärztlichen Therapie bilden die drei Verfahren Lasertherapie, intravitreale Injektion von Medikamenten und Vitrektomie. Bei der Lasertherapie werden gezielt Fotorezeptoren in der Peripherie der Netzhaut verödet, um das aus dem Gleichgewicht geratene Sauerstoffverhältnis wieder auszugleichen und die Entstehung von Neovaskularisationen zu verhindern. Ziel dieser Behandlung ist der Erhalt des Sauerstoffangebotes im Zentrum (Makula) auf Kosten einer gesenkten peripheren Rezeption. Welche Laserstrategie verwendet wird, hängt davon ab, welche Art der Retinopathie vorliegt. Bei einem Makulaödem kann fokal koaguliert werden, bei der PDR wird die Netzhautperipherie behandelt. Die zweite Säule in der Behandlung einer diabetischen Retinopathie stellt die intravitreale operative Medikamenteneingabe (IVOM) dar. Hierbei können Therapeutika direkt in den Glaskörper eingebracht werden, um dort ihre Wirkung zu entfalten. Im Speziellen handelt es sich zumeist um Anti-VEGF-Präparate, welche durch Blockierung dieses Faktors für einen Rückgang der Neovaskularisationen sorgen. So kann die IVOM von VEGFInhibitoren zusätzlich das Risiko einer durch instabile Gefäße ausgelösten Glaskörperblutung mindern. Die Effektivität und Sicherheit der neueren Präparate, wie Ranibizumab und Aflibercept, konnten in multiplen Studien nachgewiesen werden (Massin et al. 2010; Nguyen et al. 2010; Do et 
al. 2012; Schmidt-Erfurth et al. 2014). Aber auch das off-label-Präparat Bevacizumab ist sicher und wirkungsvoll (Stellungnahme der Deutschen opthalmologischen Gesellschaft). Patienten profitieren in diesem Zusammenhang am meisten von einer regelmäßigen intravitrealen Gabe des Wirkstoffes. Optional besteht auch die Möglichkeit der Injektion von Steroiden, welche die Freisetzung von angioproliferativ und inflammatorisch wirksamen Zytokinen unterbinden sollen. Diese Therapieoption stellt im klinischen Alltag jedoch eine Reserveoption dar, da sie ein ungünstigeres Nebenwirkungsprofil aufweist als die Injektion von Anti-VEGF-Präparaten. Aufgrund des erhöhten Risikos einer Glaskörperabhebung und konsekutiver Netzhautablösung bedingt durch in den Glaskörper vorwachsende Gefäße kann die Vitrektomie als dritte Therapieoption bei der Behandlung einer diabetischen Retinopathie in Betracht kommen. Die Wahl der Therapie richtet sich zunächst danach, ob eine PDR oder NPDR vorliegt. An zweiter Stelle steht die Beurteilung einer möglichen Foveabeteiligung.

Die NPDR im milden bzw. mäßigen Stadium muss in der Regel nicht behandelt werden. Patienten mit einer fortgeschrittenen NPDR mit klinischem Makulaödem ohne Beteilung der Fovea können von einer fokalen Laserkoagulation profitieren (Haritoglou et al. 2015). Im Falle einer fovealen Mitbeteiligung sollte eine IVOM-Behandlung begonnen werden.

Die panretinale Laserkoagulation eignet sich zur Behandlung einer PDR, bei der in der gesamten Netzhaut sowohl geschädigte als auch gesunde Areale verödet werden, um den Sauerstoffbedarf in der Retina zu senken. Zusätzlich kann das Risiko für eine Glaskörperblutung zum Teil erheblich reduziert werden (Evans et al. 2014). Neuere Lasermethoden umfassen hier u.a. das Prinzip der navigierten Laserbehandlung, bei der mithilfe des sogenannten eye trackings die genaue Behandlung von Leckageorten möglich gemacht wird (Kozak et al. 2011).

Eine Übersicht über die möglichen Optionen in der Therapie der diabetischen Retinopathie bietet Abbildung 8. 

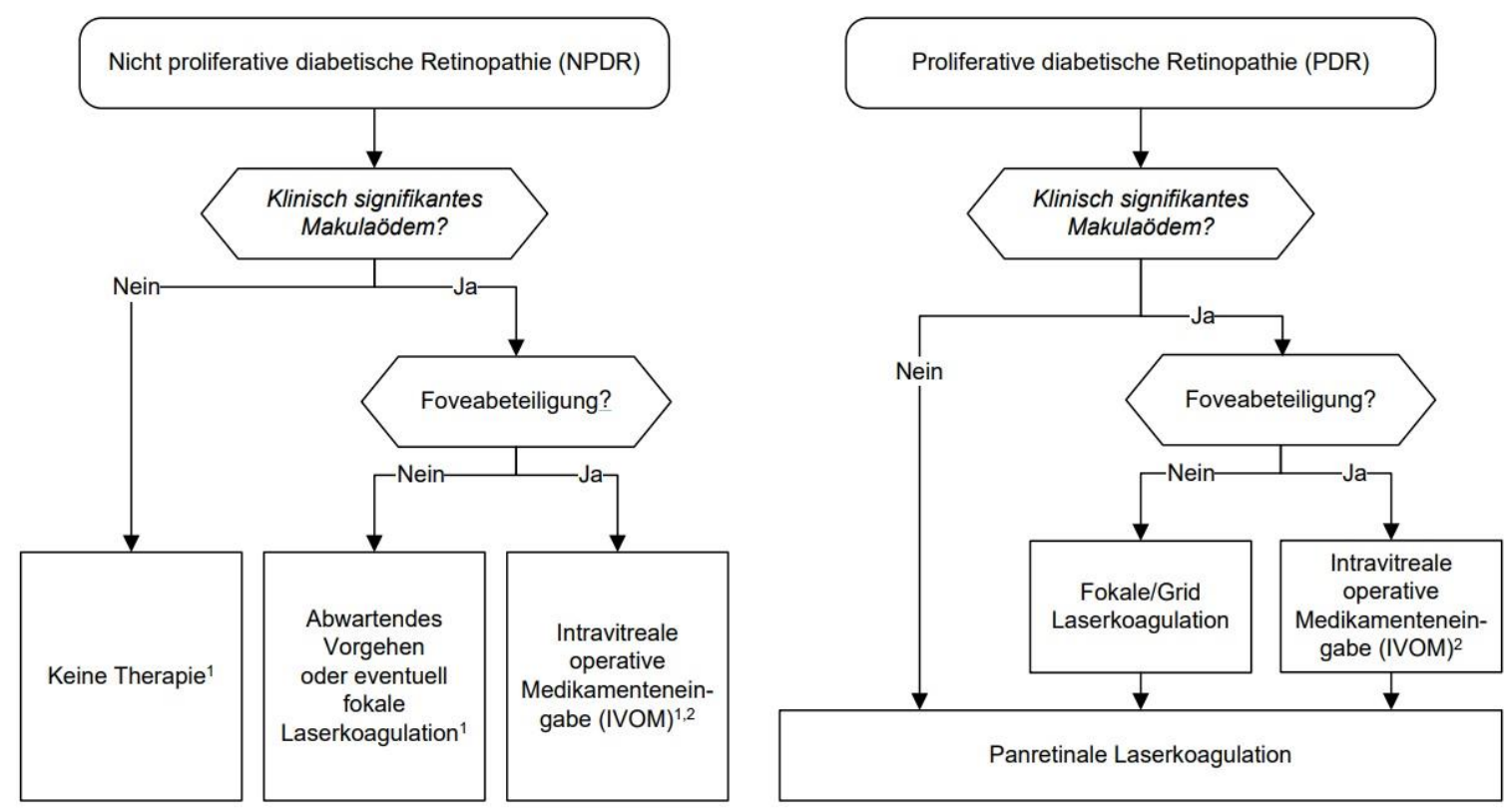

Abbildung 8: Behandlung der diabetischen Retinopathie (Bundesärztekammer (BÄK) et al. 2015), (C) ÄZQ, BÄK, KBV und AWMF 2015 (Quelle: Nationale Versorgungsleitlinie zur Prävention und Therapie von Netzhautkomplikationen bei Diabetes, die Verwendung erfolgt mit freundlicher Genehmigung durch die ÄZQ) 


\subsection{Zielsetzung und wissenschaftlicher Hintergrund}

Ziel der vorliegenden Studie ist es, eine Korrelation zwischen morphologischen und funktionellen Parametern bei diabetischen Makulaerkrankungen zu untersuchen. Die funktionellen Messergebnisse sämtlicher Untersuchungen sollten mit dem morphologischen Bild korreliert werden, um Zusammenhänge zwischen dem Grad der Ischämie und der erhaltenen Sehfunktion auf den Prüfstand zu stellen. Es wurden Korrelationsanalysen zwischen der Sehkraft (Visus und Kontrastvisus), Ausmaß und Einschränkung des Gesichtsfeldes (Perimetrie) sowie der Schichtdicke der Retina (OCT) durchgeführt. Des Weitern wurde die Krankengeschichte des Patienten mit besonderem Augenmerk auf die diabetes-bezogene Anamnese erfasst und ebenfalls mit dem Grad der Ischämie korreliert.

Bei bestehender diabetischer Retinopathie konnten sowohl quantitative als auch qualitative Veränderungen der fovealen avaskulären Zone (FAZ) gegenüber Kontrollgruppen verzeichnet werden. In Augen mit diabetischer Retinopathie (siehe Abbildung 10) kann die FAZ größer und weniger scharf begrenzt sein als bei gesunden Augen (siehe Abbildung 9), was auf die auftretende Ischämie zurückzuführen ist (Mansour et al. 1993).

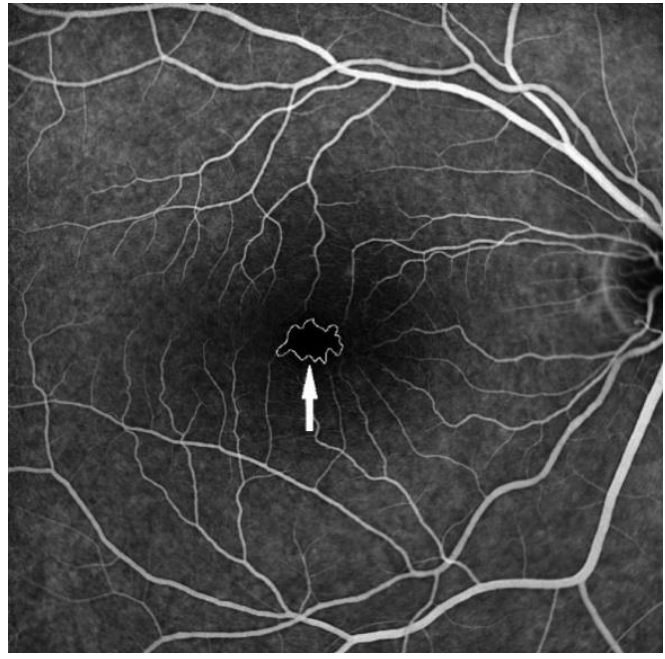

Abbildung 9: Mittlere Phase einer Fluoreszeinangiografie einer gesunden Netzhaut und normwertiger FAZ (umfahren, Pfeil) (mit freundlicher Genehmigung der Augenklinik der UMG)

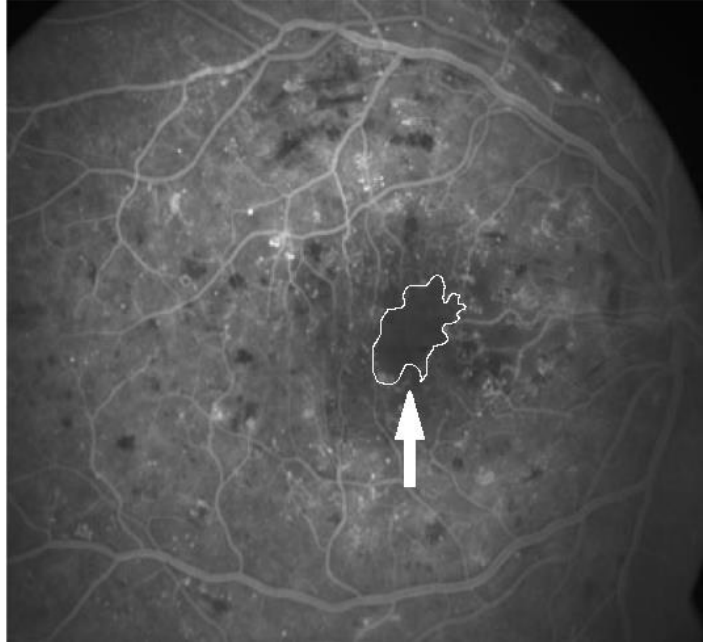

Abbildung 10: Mittlere Phase einer Fluoreszeinangiografie bei diabetischer Makulopathie, Vergrößerung der FAZ (umfahren, Pfeil) und Verlust des perifovealen Kapillarnetzes (mit freundlicher Genehmigung der Augenklinik der UMG)

Allerdings ist die genaue Bedeutung der Makulaischämie und ihrer Auswirkung auf die Sehfunktion weiterhin unklar. Verschiedene Arbeitsgruppen haben mittels Fluoreszeinangiografie bei mehr als 
50\% der Patienten mit einer diabetischen Makulopathie ischämische Anteile beobachtet. Eine herabgesetzte Sehkraft wurde jedoch erst ab fortgeschrittenen Stadien der Makulopathie und bei ausgedehnteren Ischämiearealen beschrieben (Sim et al. 2013). Es ist bisher ebenfalls unklar, ab welcher Größe der Ischämiefläche die Sehfunktion klinisch relevant beeinträchtigt ist und ob eine Behandlung eines eventuell gleichzeitig bestehenden Makulaödems noch sinnvoll ist. Außerdem ist eine fortschreitende diabetische Makulopathie prädiktiv für den Verlust der Sehkraft, jedoch ist nicht bekannt, wie schnell die diabetische Makulaischämie fortschreitet und mit welchen Faktoren dies korreliert (Sim et al. 2013). 


\section{Patienten und Methoden}

\subsection{Studiendesign}

Bei der vorliegenden Studie handelt es sich um eine monozentrische und prospektive Beobachtungsstudie an einer konsekutiv rekrutierten Fallserie. Entsprechend der Ein- und Ausschlusskriterien wurden sowohl Patienten aus der Ambulanz der Augenklinik als auch der Diabetes-Ambulanz der Universitätsmedizin Göttingen rekrutiert. Das Forschungsvorhaben wurde von der Ethikkommission der Universitätsmedizin Göttingen am 04.11.2015 genehmigt (DOK_318_2015). Der Zeitraum der Rekrutierung bemaß sich von November 2015 bis Juni 2016. Die geplante Patientenanzahl betrug 40 .

Die Patienten wurden nach ärztlicher Aufklärung einmalig in der Poliklinik der Augenklinik untersucht. Von jedem Patienten wurde nur ein Auge für die Studie eingeschlossen.

\subsection{Patientenkollektiv}

\subsubsection{Einschlusskriterien}

Die Patienten mussten zur Teilnahme an der Studie mindestens 18 Jahre alt sein und einen Diabetes mellitus Typ-1 oder Typ-2 aufweisen. Vor studienspezifischen Untersuchungen wurden die Patienten über die Studie informiert und aufgeklärt. Falls die Patienten bereit waren, an der Studie teilzunehmen, mussten sie eine Einverständniserklärung unterschreiben und erst danach wurden die Untersuchungen durchgeführt, die im Rahmen der Studie erforderlich waren.

\subsubsection{Ausschlusskriterien}

Folgende Merkmale stellten Ausschlusskriterien der Studie dar:

Tabelle 3: Ausschlusskriterien

\begin{tabular}{|l|}
\hline Ausschlusskriterien \\
\hline Fehlendes Einverständnis bzw. Einwilligungsunfähigkeit \\
\hline Getrübte optische Medien, ohne Möglichkeit der Fundus- bzw. Makulabeurteilung \\
\hline Eine bekannte Allergie gegen den Farbstoff Fluoreszein \\
\hline Ein bestehendes Makulaödem \\
\hline Vorliegen weiterer Augenerkrankungen wie retinale Gefäßverschlüsse, Glaukom, klinisch \\
\hline
\end{tabular}


relevante epiretinale Gliose, durchgreifendes Makulaforamen, altersabhängige Makuladegeneration, Uveitis posterior und Vernarbungen im Bereich der Makula

Patienten, die aufgrund eines bestehenden Makulaödems behandelt wurden, konnten in die Studie aufgenommen werden, sobald das Makulaödem nicht mehr vorlag.

\subsection{Untersuchungen, Datenerhebung und -verarbeitung}

Es wurden folgende nichtinvasive Untersuchungen einmalig durchgeführt:

Tabelle 4: Untersuchungen und Datenerhebung

\begin{tabular}{|l|}
\hline Untersuchungen und Datenerhebung \\
\hline Standardisierte Anamnese \\
\hline Refraktionsbestimmung (sphärisches Äquivalent) \\
\hline Visus- und Kontrastvisusbestimmung \\
\hline Gesichtsfeldmessung $\left(30^{\circ}\right)$ \\
\hline Spectral-Domain Optische Kohärenztomografie (SD-OCT) \\
\hline Fundusfotografie und Fluoreszeinangiografie (FA) \\
\hline Untersuchung des vorderen und hinteren Augenabschnittes in Mydriase \\
\hline
\end{tabular}

Die Daten wurden einmalig bei Patientenvorstellung erhoben und statistisch analysiert. Als Annahme bezüglich der Testung auf Signifikanz ( $p$ ) wurde die Nullhypothese festgelegt, dass zwischen der Größe der Ischämiefläche und den Ergebnissen der weiteren visuellen Untersuchungen kein statistischer Zusammenhang besteht. Ziel der Studie und der statistischen Analyse bestanden darin, diese Annahme zu widerlegen. Das Signifikanzniveau wurde für $p \leq 0,05$ festgelegt, die Berechnung erfolgte mittels t-Test.

Die Daten wurden sowohl individuell als auch in Gruppen ausgewertet. Die Gruppenanalysen bestanden aus einer Quartil- und einer Schwellenwert-Analyse. Um die Quartile bzw. Gruppen zu begrenzen, wurde bei beiden Analyse-Methoden die Unterteilung der Patientendaten anhand der gemessenen ischämischen Fläche durchgeführt. Bei der Schwellenwert-Analyse wurden die Patientendaten in zwei Gruppen unterteilt. Für eine normal große FAZ gelten Normwerte zwischen 0,3 und 0,4 $\mathrm{mm}^{2}$, der Schwellenwert wurde darauf bezogen auf 0,35 $\mathrm{mm}^{2}$ festgelegt. Die entstandenen Gruppen stellten so sowohl ein Patientenkollektiv mit wenig bis keiner (G1), als auch ein Kollektiv mit mäßiger bis ausgeprägter Ischämie (G2) dar. So ließ sich vergleichen, ob die Größe 
der FAZ bzw. der Grad der Ischämie im Bereich der Makula einen signifikanten Einfluss auf beispielsweise die Sehkraft oder die Größe des Gesichtsfelds hat. Die Aufteilung der Quartile erfolgte nach einer Ranglistenaufstellung der Daten vom kleinsten zum größten Wert. Zur Auswertung wurde die Gruppe mit den neun kleinsten Werten (Q1) mit der Gruppe der neun größten Werten (Q3) verglichen.

Im Anschluss an die Gruppenanalysen wurde eine Regressionsanalyse durchgeführt, bei der der Zusammenhang zwischen der Größe der ischämischen Fläche und den zusätzlich erhobenen Messergebnissen im Rahmen einer Korrelationstestung untersucht wurde. So ließ sich untersuchen, inwieweit zwischen zwei Variablen ein Zusammenhang besteht. Im Rahmen der Studie sollte primär festgestellt werden, ob und in welchem Ausmaß die Größe der FAZ mit den anderen untersuchten Parametern zusammenhängt.

Durchgeführt wurde die statistische Auswertung mithilfe des Programms Statistical Analysis Software (SAS). Für die Regressionsanalysen wurde der parametrische Korrelationskoeffizient nach Pearson als Maß für den statistischen Zusammenhang verwendet. Zusätzlich wurde jeweils der nicht-parametrische Korrelationskoeffizient nach Spearman angegeben, der für die Rangkorrelation verwendet wird. Er bezieht sich nicht auf die tatsächlich gemessenen Werte, sondern auf die Unterteilung der Werte nach Rängen. Dadurch ist er, im Gegensatz zu Pearson, weniger empfindlich gegenüber Ausreißern und kann auch bei nicht-normalverteilten Stichproben verwendet werden. Die Unterteilung kann jedoch zum Informationsverlust führen, da in der Analyse die tatsächlich gemessenen Werte nicht mehr auftauchen. Zur Veranschaulichung von Zusammenhängen zwischen klinisch relevanten Informationen ist die Regressionsanalyse unter Verwendung des Korrelationskoeffizienten nach Pearson besser geeignet.

Untersucht wurde bei jedem Patienten nur ein Auge. Als Studienauge wurde immer dasjenige ausgewählt, welches in der Fluoreszeinangiografie die geringere Makula-Schädigung aufwies.

Unterstützt wurde die statistische Auswertung durch Herrn Sebastian Pfeiffer vom Institut für Medizinische Statistik der UMG, AG Biometrie und Studiendokumentation.

\subsubsection{Krankheitsspezifische Anamnese}

Alle Patienten wurden nach Diabetestyp, Blutzuckerwerten, Therapie und Krankheitsdauer befragt. 


\subsubsection{Sphärisches Äquivalent}

Erhoben wurden diese Werte am Autorefraktometer ARK-1S der Firma Nidek. Der Patient fixierte dazu durch ein Okular ein Bild im Inneren des Geräts. Es wurde Messlicht auf die Netzhaut des Auges gerichtet und von dort reflektiert. Abhängig von der jeweiligen Fehlsichtigkeit des Patienten wurde das Licht unterschiedlich zurückgeworfen und vom System registriert. Die Dokumentation der Untersuchung erfolgte mittels der standardisierten Untersuchungsbögen der UMG (siehe Anhang).

\subsubsection{Visus- und Kontrastvisusbestimmung}

Der Visus wurde nach Linsenkorrektur mithilfe der ETDRS-Tafel bestimmt (ETDRS Visual Acuity Score, ETRDS-VAS). Diese ist in Bezug auf Entfernung des Patienten zur Tafel, Beleuchtung, Abstand der Zeilen etc. standardisiert und ermöglicht so gute Handhabbarkeit vor allem für Studien. Die Tafel zeigt 14 Reihen mit jeweils fünf Buchstaben, die in logarithmischer Abstufung kleiner werden (siehe Abbildung 11).

Die Untersuchung wurde aus einer Entfernung von vier Metern durchgeführt. Die Tafel wurde vor einem Illuminator präsentiert, der die Optotypen von hinten beleuchtet. Der Patient wurde gebeten die Optotypen Zeile für Zeile widerzugeben. Konnte der Patient in einer Zeile weniger als vier von fünf Buchstaben richtig erkennen, wurde die Untersuchung beendet. Die Anzahl der richtig erkannten Optotypen wurde addiert, konnte der Patient 20 oder mehr Optotypen korrekt widergeben, wurden zusätzlich 30 Punkte zum Ergebnis addiert. Es war eine Maximalpunktzahl von 100 erreichbar. 


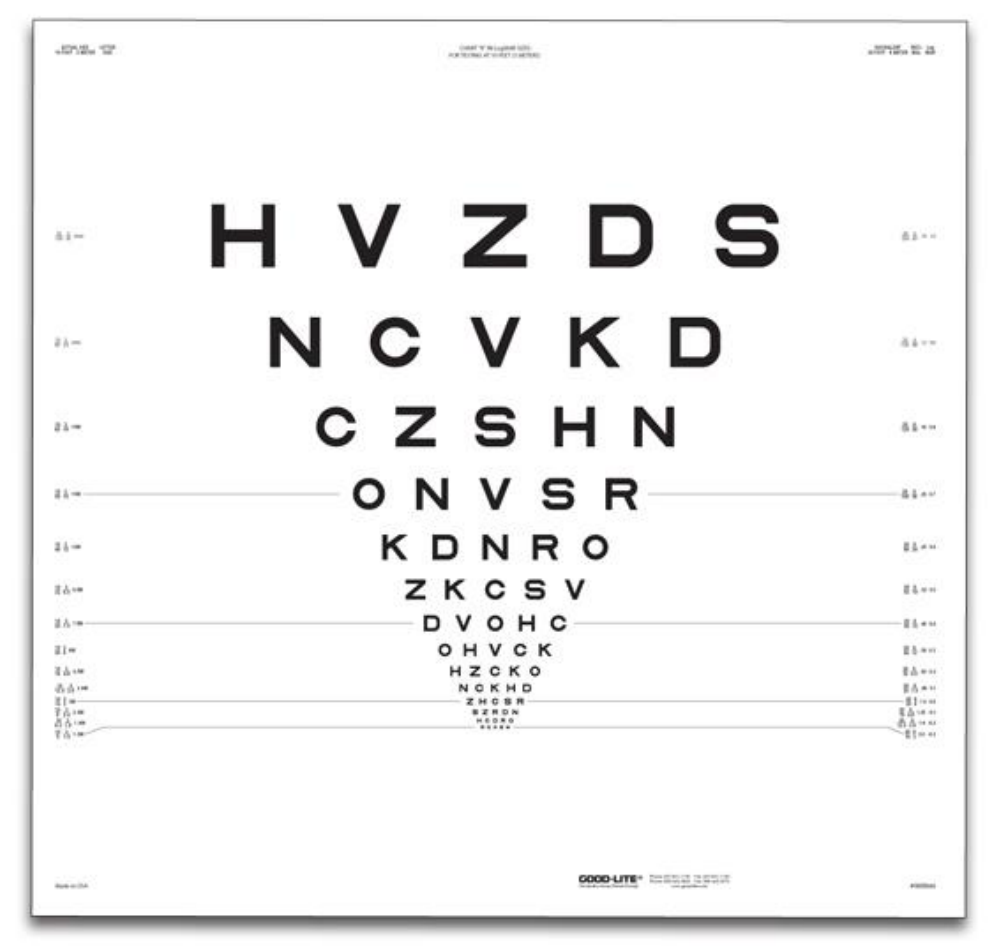

\section{Abbildung 11: ETDRS-Tafel (Quelle: Trusetal Verbandstoffwerk GmbH mit freundlicher Genehmigung durch den Hersteller)}

Zur Überprüfung des Kontrastsehens wurden dem Patienten Sehzeichen präsentiert, die sich sowohl in Kontrast als auch in Größe unterschieden. Für die Studie wurde das Bailey-Lovie-Chart-Set verwendet, welches sich für die Verwendung in Studien zur Bestimmung der Sehkraft in hohem und niedrigem Kontrast bewährt hat. So bot diese Form der Untersuchung ein hohes Maß an Vergleichbarkeit. Die Durchführung erfolgte in der gleichen Weise, wie die des normalen Visus, jedoch musste kein fester Abstand des Patienten zur Tafel eingehalten werden. Dem Probanden wurden zwei Tafeln mit je 11 Reihen à fünf Buchstaben präsentiert, auf denen sich die Optotypen in ihrem Kontrast zum weißen Hintergrund unterschieden. Eine Tafel wieß einen hohen Kontrast mit schwarzen Optotypen auf weißem Grund auf. Die zweite Tafel zeigte Optotypen in schwächerem Kontrast zum Hintergrund. Diese Untersuchung wurde ohne die Hilfe eines Illuminators durchgeführt. Sowohl bei der Erhebung des high-contrast- als auch des low-contrast-Visus war eine Maximalpunktzahl von 55 möglich, welche sich aus der korrekten Benennung der einzelnen Optotypen ergab. Die Ergebnisse beider Visusuntersuchungen wurden auf den standardisierten Untersuchungsbögen der Universitätsmedizin Göttingen (siehe Anhang) dokumentiert. 


\subsubsection{Gesichtsfeldmessung}

Im Rahmen der Studie wurde die statische Perimetrie gemessen. Sie wurde am OCTOPUS ${ }^{\circledR} 900$ der Firma Haag-Streit Diagnostics durchgeführt. Es wurde das Programm M2X gewählt, bei dem nur die zentralen $12^{\circ}$ des Gesichtsfeldes analysiert werden, da sich dies besonders gut für die Beurteilung der Makula eignet. Es wurden bei jedem Patienten 81 Prüfpunkte getestet, die sich in vier Quadranten mit jeweils 20 Punkten und einem gemeinsamen Mittelpunkt aufteilen (siehe Abbildung 12).
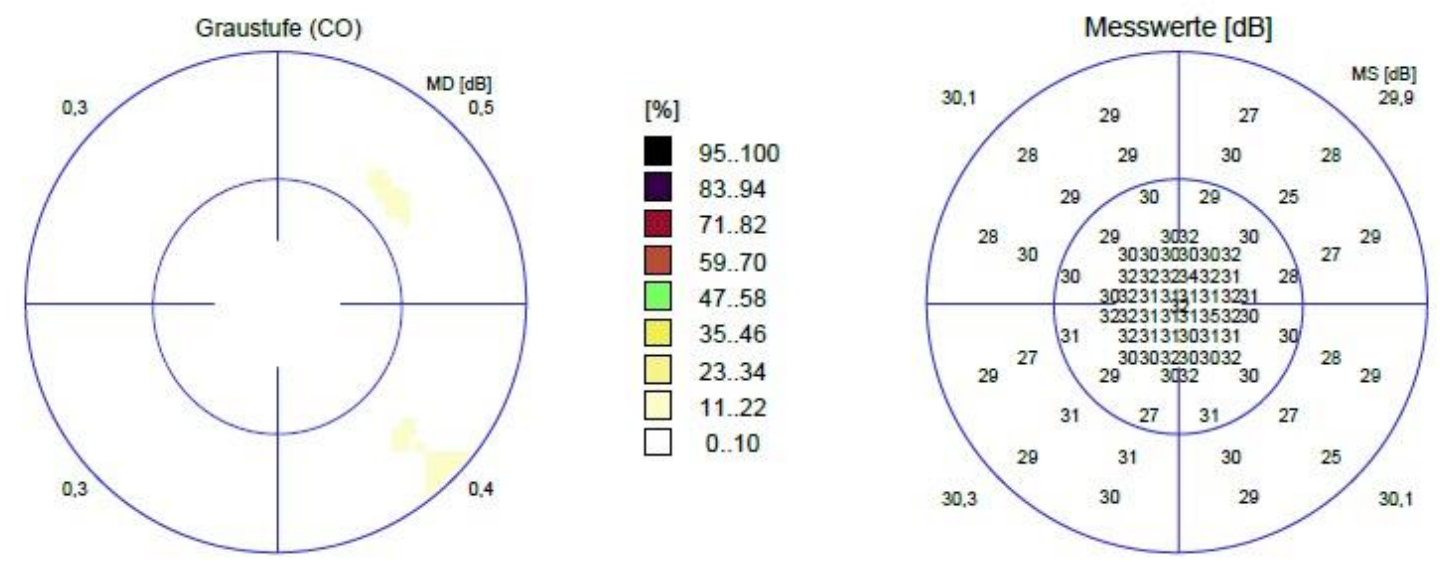

Abbildung 12: Beispiel einer perimetrischen Untersuchung mittels Octopus 900, links MD [dB] Verteilung in Graustufen, rechts MS [dB] Verteilung der reinen Messwerte (mit freundlicher Genehmigung der Augenklinik der UMG)

Analysiert wurden im Rahmen der Studie die beiden Gesichtsfeldindizes mean sensitivity (MS) und mean defect (MD) in Dezibel (dB). Es handelt sich hierbei um so genannte globale Indizes in Bezug auf die Gesichtsfeldmessung, die aus den gemessenen Empfindlichkeitswerten errechnet wurden. Bei der MS handelt es sich um die mittlere Empfindlichkeit, also den Mittelwert aller gemessenen Schwellenwerte, bezogen auf eine Gesichtsfeldfläche. Zur Berechnung des MD wurden die erhobenen Werte mit den Normwerten einer gleichaltrigen Kohorte verglichen und ebenfalls ein Mittelwert gebildet. Die Differenz zwischen dem gemessenen Wert des Patienten und dem Normalwert für die Alterskohorte und die gemessene Lokation bildete den "Defekt" ab. War die zentrale Retina des Patienten empfindlicher, als es für seine Altersgruppe üblich gewesen wäre, ergab sich durch die Bildung der Differenz ein negativer Wert. Ein negatives Vorzeichen kennzeichnete eine höhere, ein positives Vorzeichen eine geminderte Empfindlichkeit auf einen präsentierten Lichtreiz im Vergleich zur Alterskohorte. Für den MD war eine Toleranzbreite von -2 $\mathrm{dB}$ bis $+2 \mathrm{~dB}$ voreingestellt. Die berechneten Defektwerte waren aufgrund der bereits erfolgten Korrelation unabhängig vom Alter des Patienten oder der Messlokalisation (siehe Abbildung 13). 

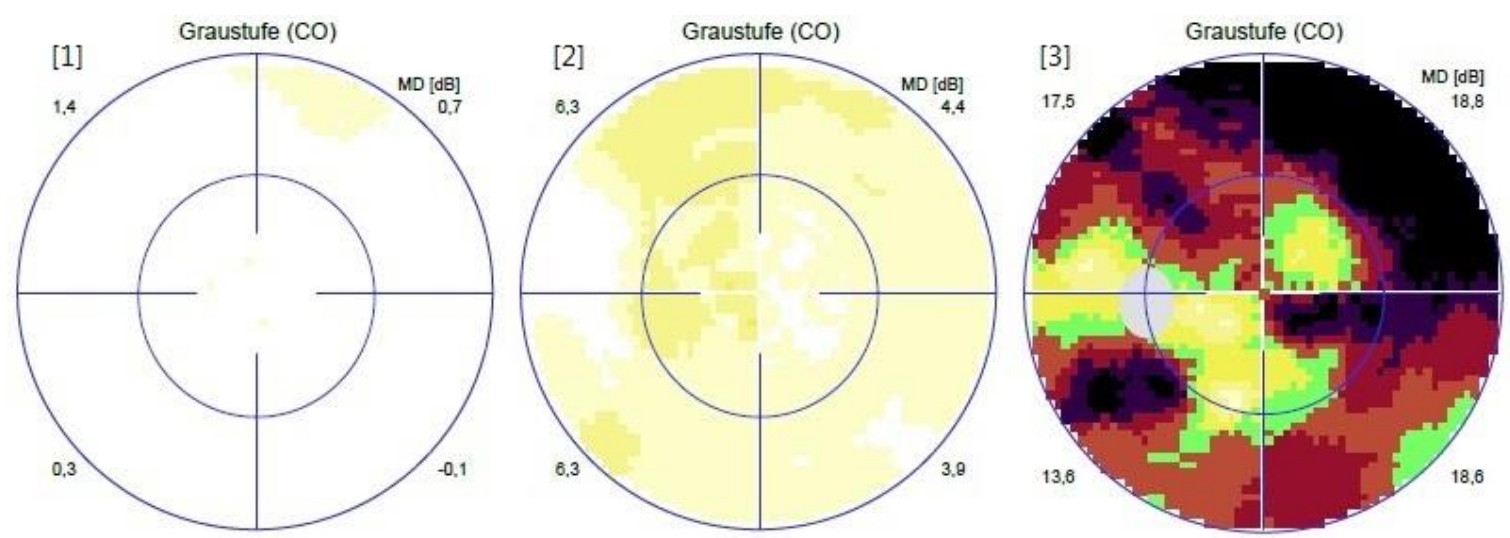

Abbildung 13: Beispiele Defektstärken für geringen [1], mäßigen [2] und ausgeprägten Defekt [3], CODarstellung, Octopus 900 (mit freundlicher Genehmigung der Augenklinik der UMG)

Zusätzlich wurden die Anteile der gestellten Fangfragen ausgewertet. Gab ein Patient an, einen nicht sichtbaren Lichtreiz trotzdem gesehen zu haben, wurde dies als "falsch-positive" Antwort gewertet. Über die gesamte Untersuchung wurden alle dargebotenen Reize gezählt, der Anteil der "falsch-positiven" Fangfragen berechnet und im Befund angegeben. Neben den "falschpositiven" Fangfragen wurden zusätzlich "falsch-negative" Reize präsentiert. Sie dienen dazu festzustellen, ob ein Patient angibt, weniger oder nur intensivere Reize zu sehen, als eigentlich möglich wären. Zu diesem Zweck werden dem Patienten an Lokalisationen, die bereits getestet wurden und einen bekannten Schwellenwert aufweisen, erneut Lichtreize präsentiert, die eine weitaus höhere Intensität aufweisen, als die Schwelle. Wird dieser Reiz nicht mehr erkannt, wird er als "falsch-negativ" gewertet. Auch der Anteil dieser Fangfragen wird als prozentualer Wert im Befund angegeben.

\subsubsection{Optische Kohärenztomografie (OCT)}

Für die Studie wurden die OCT-Messungen am SPECTRALIS ${ }^{\circledR}$ OCT der Firma Heidelberg Engineering durchgeführt. Es wurden vier unterschiedliche Bereiche der Makula untersucht (siehe Abbildung 14). Die Benennung erfolgte standardmäßig nach der Lage der einzelnen Quadranten:

- center point (CP) - der Mittelpunkt, an dem sich alle eingezeichneten Linien treffen

- central subfield (CSF) - der Bereich, der sich im Radius von $1 \mathrm{~mm}$ um den center point befindet 
- inner subfield (ISF) - gliedert sich in vier Bereiche, die nach der jeweiligen Lage im Raum benannt werden (superior, nasal, inferior und temporal), liegt im Radius $500-1500 \mu \mathrm{m}$ um den $\mathrm{CP}$ herum

- outer subfield (OSF) - wird ebenfalls in vier Bereiche gegliedert, die Benennung erfolgt in gleicher Weise wie für das ISF, liegt im Radius von 1500 - 3000 um um den CP herum

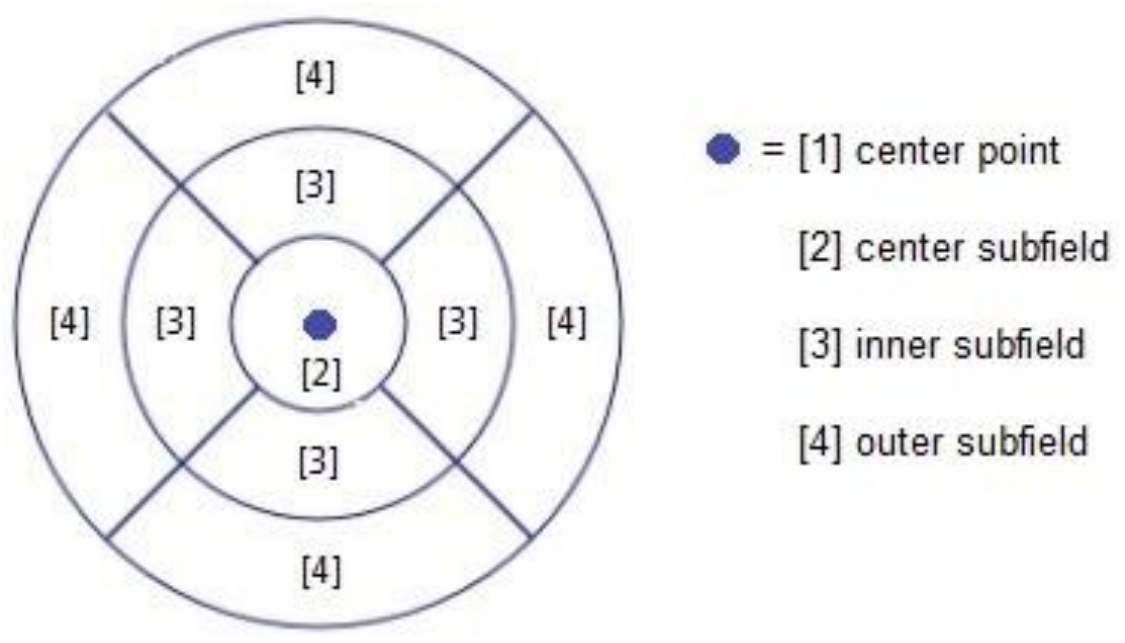

Abbildung 14: Bereiche der OCT-Messung, Schema (mit freundlicher Genehmigung der Augenklinik der UMG)

Für jeden Bereich wurden Volumina und die Dicke einzeln berechnet (siehe Abbildung 15).
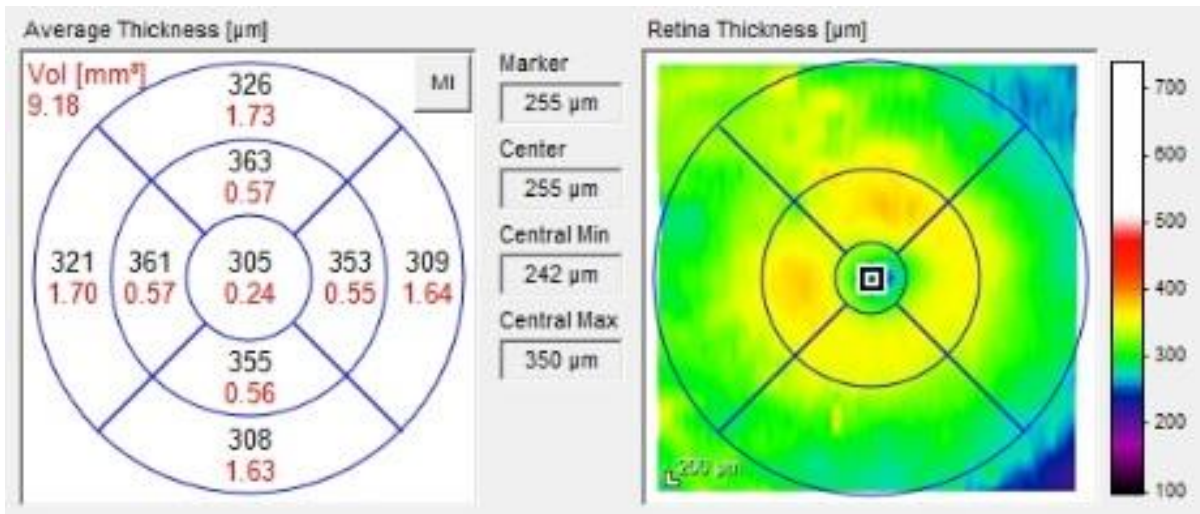

Abbildung 15: Beispiel einer OCT-Messung in Absolutwerten- [links] und Graustufendarstellung [rechts] (mit freundlicher Genehmigung der Augenklinik der UMG)

Es wurden die Dickenmessungen für den center point (CPT) und das center subfield (CSFT), sowie die Volumenbestimmungen für die gesamte Makula (TMV) und das center subfield (CSFV) bei jedem 
Patienten bestimmt und im Anschluss analysiert. Es wurde ein Scanfeld mit von $6 \mathrm{~mm} \times 6 \mathrm{~mm}$ $\left(20^{\circ} \times 20^{\circ}\right)$, zentriert auf die Fovea, mit parallel angeordneten B-Scans verwendet (siehe Empfehlung der Makulakommission vom März 2017).

\subsubsection{Fundusfotografie und Fluoreszeinangiografie}

Für die Studie wurden die Fotos sowie die Fluoreszeinangiografie an der Funduskamera FF450 plus IR der Firma Zeiss aufgenommen. Es wurden zuerst Bilder ohne und anschließend mit Farbstoff angefertigt. Verwendet wurde $3-5 \mathrm{ml}$ fluoreszierendes Kontrastmittel, welches über fünf Sekunden appliziert wurde. Es wurden nach einer Minute, fünf und zehn Minuten Aufnahmen angefertigt. Um den Grad der Ischämie zu bestimmen, wurde die Fläche der FAZ auf den Aufnahmen der Fluoreszeinangiografie ausgemessen. Die Auswertung der Aufnahmen der Fluoreszeinangiografie erfolgte durch zwei Retinologen der UMG, im Folgenden als Untersucher 1 (U1) und Untersucher 2 (U2) bezeichnet. Die Bilder aus den Untersuchungen wurden zur Bestimmung der Größe der FAZ angefertigt.

Die Fluoreszeinangiografie stellt die zentrale Untersuchung der vorliegenden Studie dar. Bezogen auf die Ergebnisse dieser Untersuchung erfolgte die Unterteilung der Gruppen für die unterschiedlichen Analyse-Typen (siehe Kapitel 2.3). Für die Auswertung wurde die Software „Image J“ verwendet. Mithilfe einer Indexlinie einer bestimmten Länge für jede FA-Aufnahme wurde die Größe der FAZ für jeden Patienten errechnet. Die Abmessung wurde von jedem Untersucher unabhängig einmal durchgeführt. Die doppelte Ausmessung soll als Absicherung dienen, da die Bestimmung von Größe und Lage der FAZ rein auf Helligkeitsunterschieden in der Fluoreszeinangiografie-Aufnahme beruht. Eine automatische Erkennung der genauen Lokalisation ist im Rahmen dieser Untersuchungsmethode nicht möglich und es kann somit untersucherabhängig zu geringfügig unterschiedlichen Messwerten bezüglich der exakten Größe kommen. Für die Gruppenaufteilung und Korrelationsanalysen wurde jeweils der gebildete Mittelwert der beiden gemessen Größen verwendet. 


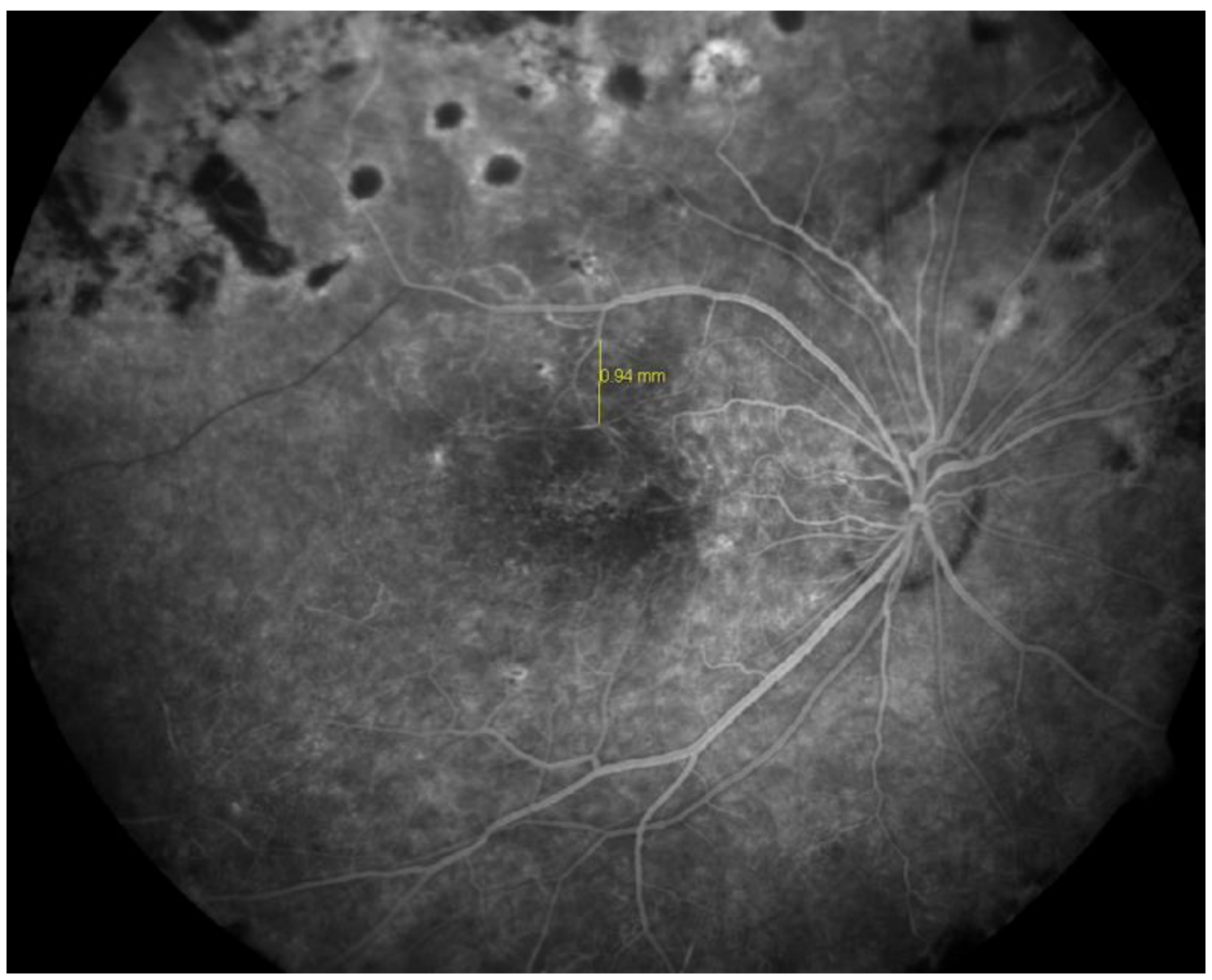

Abbildung 16: Beispiel einer Abmessungsbestimmung (hier: Abstand zwischen zwei willkürlich gewählten Gefäßen 0,94 mm) (mit freundlicher Genehmigung der Augenklinik der UMG) 


\section{Ergebnisse}

\subsection{Demografie}

In die prospektive Studie wurden insgesamt 40 konsekutive Patienten eingeschlossen. Es konnten nicht bei jedem Patienten alle Parameter erhoben werden, da zum Teil Untersuchungsgeräte zum Zeitpunkt des Patientenbesuchs nicht verfügbar waren. Vereinzelt waren Messungen nicht auswertbar oder für die Studie ungeeignet, da sie in anderen Geräten oder Untersuchungsmodi durchgeführt worden waren. Von 40 in die Studie eingeschlossenen Patienten ist bei 36 die korrekte Fluoreszeinangiografie wie geplant an der VISUCAM gelaufen und konnte ausgewertet werden. Anhand dieser Untersuchung wurde das Gesamtpatientenkollektiv festgelegt $(\mathrm{N}=36)$, da für die Korrelationsanalyse vor allem die Auswertung der Angiografie eine zentrale Bedeutung hat.

Das mittlere Patientenalter innerhalb der Stichprobe lag bei $61 \pm 16$ Jahren $(p=0,72)$. Der jüngste Patient war zum Zeitpunkt der Untersuchung 31, der Älteste 86 Jahre alt. Es wurden sowohl Patienten mit Diabetes mellitus Typ-1, als auch Typ-2 untersucht (Verhältnis 1:4). In der Patientenkohorte befand sich ein Proband mit der Diagnose eines pankreopriven Diabetes bei Zustand nach Pankreasresektion, bei fünf Patienten konnte der Diabetes-Typ nicht bestimmt werden (siehe Tabelle 5 und Abbildung 17). Für die Verteilung der Diabetes-Typen ergab sich ein Signifikanzwert von $p=0,47$. Bei 12 Patienten wurde das linke, bei 24 Patienten das rechte Auge untersucht.

Tabelle 5: Demographie und Patienten-Charakteristika

\begin{tabular}{|l|c|c|c|c|c|c|c|c|c|}
\hline & \multicolumn{3}{|c|}{ Männlich (N = 26) } & \multicolumn{3}{c|}{ Weiblich (N = 10) } & \multicolumn{3}{c|}{ Total (N = 36) } \\
\hline & N & $\%$ & mean \pm SD & N & $\%$ & mean \pm SD & N & $\%$ & mean \pm SD \\
\hline Alter [a] & 26 & & $62 \pm 16,3$ & 10 & & $59,9 \pm 12,5$ & 36 & & $61,4 \pm 15,2$ \\
\hline Diabetes-Typ & & & & & & & & & \\
\hline Typ-1 & 3 & 11,5 & & 3 & 30 & & 6 & 16,7 & \\
\hline Typ-2 & 19 & 73,1 & & 5 & 50 & & 24 & 66,7 & \\
\hline Pankreopriv & 1 & 3,9 & & 0 & 0 & & 1 & 2,8 & \\
\hline Unbekannt & 3 & 11,5 & & 2 & 20 & & 5 & 13,9 & \\
\hline
\end{tabular}


Geschlecht Weiblich

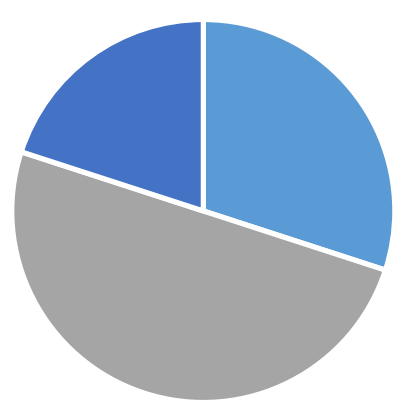

Geschlecht Männlich

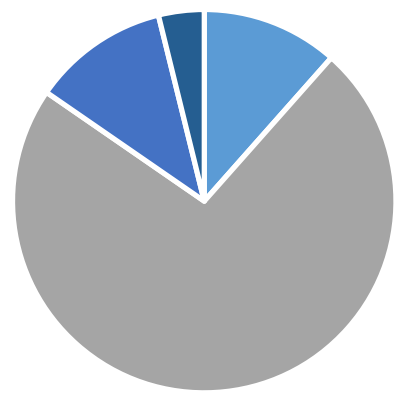

- Typ-1

- Typ-2

- Unbekannt

- Pankreopriver

Diabetes

\section{Abbildung 17: Geschlechtergetrennte Verteilung der Diabetes-Typen}

Der mittlere Langzeitzuckerwert $\left(\mathrm{HbA}_{1 c}\right.$ in \%), betrug 7,6. Dabei befand sich der Anteil an glykiertem Hämoglobin bei den Typ-1-Diabetikern rund einen Prozentpunkt unter dem der Typ-2-Diabetiker. Zum Zeitpunkt der Untersuchung lag die mittlere Erkrankungsdauer bei etwa 21 Jahren. Während die Gruppe der Typ-1-Diabetiker im Mittel etwa seit 28 Jahren erkrankt und seit ebenso langer Zeit auf die Behandlung mit Insulin angewiesen war, zeigte sich bei den Patienten mit Typ-2-Diabetes eine um 10 Jahre kürzere Erkrankungsdauer. Die Therapiedauer mit Insulin lag sogar 17 Jahre unterhalb derjenigen von Typ-1-Diabetikern (siehe Tabelle 6).

\section{Tabelle 6: Diabetesbezogene Daten}

\begin{tabular}{|l|c|c|c|c|c|c|c|c|}
\hline & $\begin{array}{c}\text { Typ-1-Diabetes } \\
\text { (N = 6) }\end{array}$ & \multicolumn{2}{|c|}{$\begin{array}{c}\text { Typ-2-Diabetes } \\
\text { (N = 24) }\end{array}$} & \multicolumn{2}{|c|}{$\begin{array}{c}\text { Pankreopriver } \\
\text { Diabetes (N = 1) }\end{array}$} & \multicolumn{4}{|c|}{ Total } \\
& N & mean \pm SD & N & mean \pm SD & N & mean \pm SD & N & mean \pm SD \\
\hline $\mathrm{HbA}_{1 \mathrm{c}}[\%]$ & 6 & $7 \pm 0,7$ & 24 & $7,7 \pm 1,6$ & 1 & 8 & 31 & $7,6 \pm 1,5$ \\
\hline Erkrankungsdauer [a] & 6 & $27,7 \pm 9,2$ & 24 & $18 \pm 8,5$ & 1 & 44 & 31 & $20,7 \pm 10,2$ \\
\hline $\begin{array}{l}\text { Therapiedauer mit } \\
\text { Insulin [a] }\end{array}$ & 6 & $27,7 \pm 9,2$ & 17 & $11,3 \pm 6,5$ & 1 & 26 & 24 & $16,8 \pm 9,8$ \\
\hline
\end{tabular}

Die Patienten wurden nach der Ischämiefläche in der Fluoreszeinangiografie in eine Gruppe von maximal $0,35 \mathrm{~mm}^{2}(\mathrm{G} 1 ; \mathrm{N}=6)$ und eine Gruppe von mehr als $0,35 \mathrm{~mm}^{2}$ Ischämiefläche (G2; $\left.N=30\right)$ eingeteilt. Beide Gruppen zeigten keinen signifikanten Unterschied hinsichtlich Durchschnittsalter und Diabetes-Typen (siehe Tabelle 7). Jeweils ein Sechstel der untersuchten Probanden litt an Typ-1Diabetes, zwei Drittel wiesen einen Typ-2-Diabetes auf. Das Durchschnittsalter lag unterhalb des Schwellenwertes (G1) etwa ein Jahr höher als oberhalb (G2) ( $p=0,89)$. In $G 1$ lag der HbA $\mathrm{H}_{1 c}$ um fast einen Prozentpunkt niedriger als in der Vergleichsgurppe $(G 2)(p=0,2)$. Die Erkrankungsdauer 
betrug etwa 4,5 Jahre weniger ( $p=0,36$ ) und auch der Behandlungszeitraum, in dem Insulin verabreicht wurde, fiel mit 9 Jahren in G1 deutlich geringer aus als in der Gruppe mit einer Ischämiefläche oberhalb des Schwellenwertes (G2) $(p=0,14)$. Es ist erkennbar, dass bei Patienten mit einer in etwa normal großen FAZ (G1) gleichzeitig ein kürzerer Erkrankungszeitraum mit weniger Jahren Insulintherapie vorlag.

Tabelle 7: Demografie und Patienten-Charakteristika [Schwellenwert-Analyse in Gruppen G1 und G2]

\begin{tabular}{|c|c|c|c|c|c|c|c|}
\hline & \multicolumn{3}{|c|}{$\leq 0,35 \mathrm{~mm}^{2}(\mathrm{G} 1)(\mathrm{N}=6)$} & \multicolumn{3}{|c|}{$>0,35 \mathrm{~mm}^{2}(\mathrm{G} 2)(\mathrm{N}=30)$} & \multirow{2}{*}{$\begin{array}{c}\text { t-Test } \\
\text { p-valu }\end{array}$} \\
\hline & $\mathbf{N}$ & $\%$ & mean \pm SD & $\mathbf{N}$ & $\%$ & mean \pm SD & \\
\hline Alter [a] & 6 & & $62,2 \pm 18,7$ & 30 & & $61,2 \pm 14,8$ & 0,89 \\
\hline Diabetestyp & & & & & & & 1 \\
\hline Typ-1-Diabetes & 1 & 16,7 & & 5 & 16,7 & & \\
\hline Typ-2-Diabetes & 4 & 66,7 & & 20 & 66,7 & & \\
\hline Pankreopriver Diabetes & 0 & 0 & & 1 & 3,3 & & \\
\hline Diabetestyp unbekannt & 1 & 16,7 & & 4 & 13,3 & & \\
\hline $\mathrm{HbA}_{1 \mathrm{c}}[\%]$ & 5 & & $6,8 \pm 0,6$ & 26 & & $7,7 \pm 1,6$ & 0,2 \\
\hline Erkrankungsdauer [a] & 5 & & $16,8 \pm 6,6$ & 26 & & $21,4 \pm 10,7$ & 0,36 \\
\hline Therapiedauer mit Insulin [a] & 3 & & $9 \pm 5,6$ & 21 & & $18 \pm 9,9$ & 0,14 \\
\hline
\end{tabular}

Die Quartilanalyse in Tabelle 8 bezüglich der Demographie zeigte, dass sich vor allem das Patientenalter $(p=0,33)$ und die Behandlungsdauer mit Insulin $(p=0,18)$ am meisten zwischen den beiden Extremgruppen unterschieden $(p=0,18)$. Im Q1-Quartil waren die Patienten rund 7 Jahre älter als in Q3 und wurden ca. 8 Jahre weniger mit Insulin behandelt. Von insgesamt 9 Patienten in jeder Gruppe wurde in Q1 weniger als die Hälfte der Patienten überhaupt mit Insulin behandelt, in Q3 zwei Drittel der Patienten. Der Langzeitzuckerwert zeigte sich im Vergleich zu Q3 in Q1 etwas höher $(p=0,44)$

Tabelle 8: Diabetesbezogene Daten [Quartilanalyse in Gruppen Q1 und Q3]

\begin{tabular}{|c|c|c|c|c|c|}
\hline & \multicolumn{2}{|c|}{$\leq 0,45 \mathrm{~mm}^{2}(\mathrm{Q} 1)(\mathrm{N}=9)$} & \multicolumn{2}{|c|}{$\geq 1,16 \mathrm{~mm}^{2}(\mathrm{Q} 3)(\mathrm{N}=9)$} & \multirow{2}{*}{$\begin{array}{c}\text { t-test } \\
p \text {-value }\end{array}$} \\
\hline & $\mathbf{N}$ & mean \pm SD & $\mathbf{N}$ & mean \pm SD & \\
\hline Alter [a] & 9 & $63 \pm 15,3$ & 9 & $55,7 \pm 15,6$ & 0,33 \\
\hline $\mathrm{HbA}_{1 \mathrm{c}}[\%]$ & 8 & $7,6 \pm 1,4$ & 8 & $7,2 \pm 0,8$ & 0,44 \\
\hline
\end{tabular}




\begin{tabular}{|l|c|c|c|c|c|}
\hline Erkrankungsdauer [a] & 8 & $15,3 \pm 6,2$ & 8 & $17,9 \pm 7,8$ & 0,49 \\
\hline $\begin{array}{l}\text { Therapiedauer mit Insulin } \\
\text { [a] }\end{array}$ & 4 & $8,8 \pm 4,6$ & 6 & $17 \pm 10,4$ & 0,18 \\
\hline
\end{tabular}

\subsection{Fundusfotografie und Fluoreszeinangiografie}

Die Abbildung 18 zeigt das Ergebnis der beiden durchgeführten Messungen der Ischämiefläche im Vergleich. Sie wurde durch zwei unabhängige Untersucher vermessen. Um nachzuweisen, dass es sich um ähnlich gemessene Werte handelt, wurden die Größen der Ischämieflächen gegeneinander auf jeweils der $\mathrm{x}$ - und der $\mathrm{y}$-Achse aufgetragen. Zwischen den beiden Größenmessungen zeigte sich ein beinah linearer Zusammenhang (Pearson-Korrelationskoeffizient 0,94; $p<0,001$ ). Die Werte korrelierten also sehr gut miteinander und es ist davon auszugehen, dass der gemittelte Wert beider Abmessungen eine realistische Repräsentation der tatsächlichen FAZ-Größe darstellt.

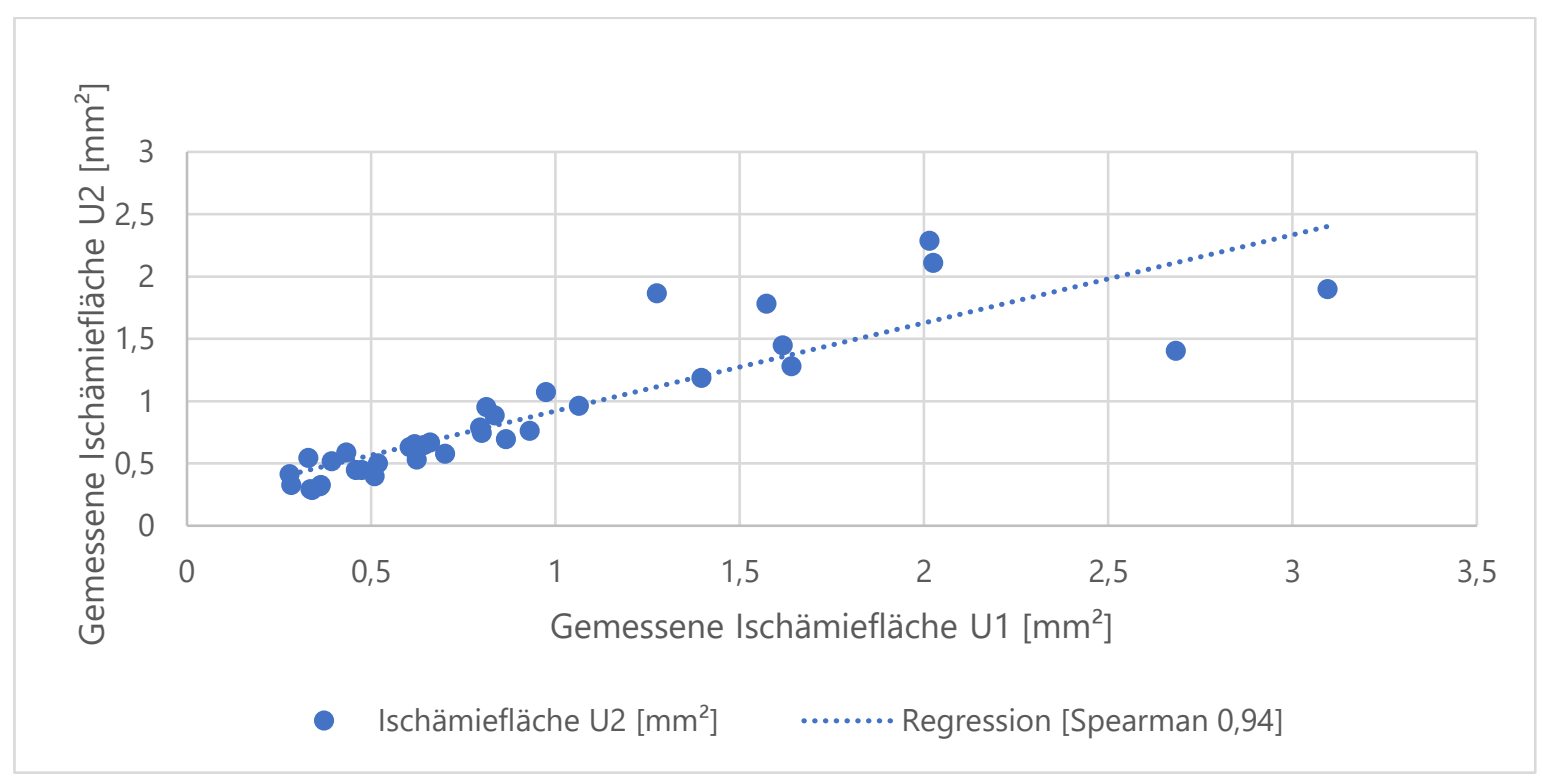

Abbildung 18: Gemessene Ischämieflächen $\left[\mathrm{mm}^{2}\right]$ von U1 und U2 anhand des FluoreszeinangiografieBildes

Die Abbildung 19 bietet einen Überblick über die Gesamtheit der gemessenen Ischämieflächen und den gemittelten Durchschnitt. Bei etwa zwei Drittel der untersuchten Kohorte (25 Patienten) lag eine durchschnittliche FAZ-Größe von unter $1 \mathrm{~mm}^{2}$ vor. Nur 11 Patienten wiesen höhere Werte auf. 


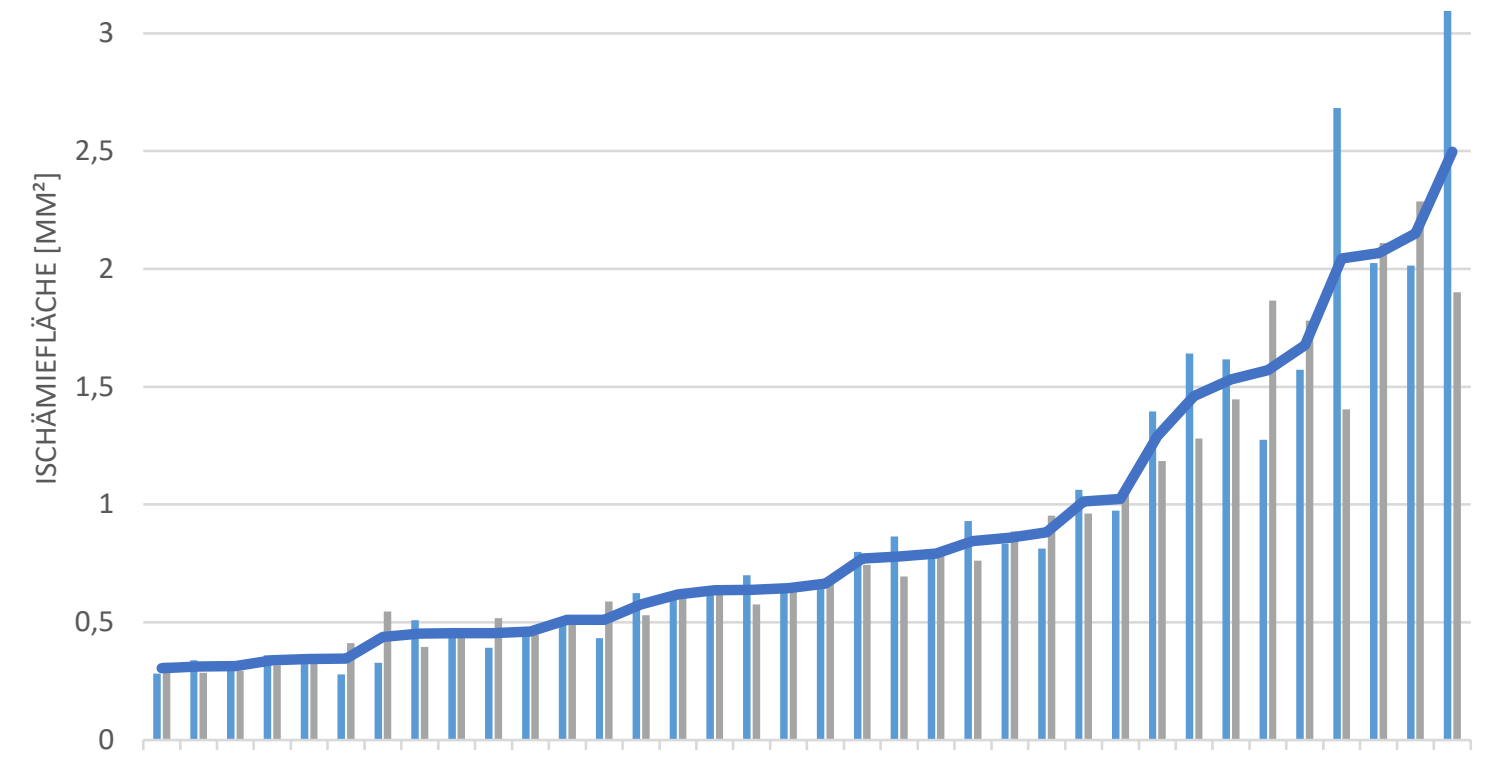

178311624182820392323723730522251540613510141333412911273842119 PATIENTEN-ID

Ischämiefläche U1 [mm²] Ischämiefläche U2 [mm²]

Gemittelte Ischämiefläche [mm²]

Abbildung 19: Übersicht über die gemessen Ischämieflächen $\left[\mathrm{mm}^{2}\right]$ im Fluoreszeinangiografie-Bild 


\subsection{Sphärisches Äquivalent}

Die Fehlsichtigkeit der Kohorte war insgesamt nicht stark ausgeprägt. Der Durchschnittswert des sphärischen Äquivalents des Patientenkollektivs lag bei $-0,21 \pm 2,15$ Dioptrien (dpt). Auch in den Gruppenanalysen fand sich stets ein geringes sphärisches Äquivalent mit Werten zwischen 0,33 dpt $\pm 2,14$ und 0,34 dpt \pm 1,75 (siehe Tabelle 9). In der Quartil-Analyse zeigte sich, dass in Q3 ein gemitteltes positives sphärisches Äquivalent gemessen werden konnte. Sowohl in Q1 als auch in beiden Schwellenwert-Gruppen lag das sphärische Äquivalent stets im negativen Bereich. Für die Quartil-Analyse lag der Signifikanzwert bei $p=0,49$ und in der Schwellenwert-Analyse bei $p=0,92$.

Tabelle 9: Sphärisches Äquivalent [Quartil-(Q1 und Q3) und Schwellenwert-Analyse (G1 und G2)]

\begin{tabular}{|c|c|c|c|c|c|c|c|c|c|c|c|c|}
\hline & \multicolumn{2}{|c|}{$\begin{array}{c}\leq 0,45 \mathrm{~mm}^{2} \\
(\mathrm{Q} 1)\end{array}$} & \multicolumn{2}{|c|}{$\begin{array}{c}\geq 1,16 \mathrm{~mm}^{2} \\
\text { (Q3) }\end{array}$} & \multirow{2}{*}{$\begin{array}{c}\text { t-test } \\
\begin{array}{c}p- \\
\text { value }\end{array}\end{array}$} & \multicolumn{2}{|c|}{$\begin{array}{c}\leq 0,35 \mathrm{~mm}^{2} \\
\text { (G1) }\end{array}$} & \multicolumn{2}{|c|}{$\begin{array}{c}>0,35 \mathrm{~mm}^{2} \\
\text { (G2) }\end{array}$} & \multirow{2}{*}{$\begin{array}{c}\text { t-test } \\
\begin{array}{c}p- \\
\text { value }\end{array}\end{array}$} & \multicolumn{2}{|c|}{$\begin{array}{c}\text { Total } \\
(\mathrm{N}=36)\end{array}$} \\
\hline & $\mathbf{N}$ & $\begin{array}{c}\text { mean } \pm \\
\text { SD }\end{array}$ & $\mathbf{N}$ & $\underset{\text { SD }}{\operatorname{mean}} \pm$ & & $\mathbf{N}$ & $\underset{\text { SD }}{\operatorname{mean}} \pm$ & $\mathbf{N}$ & $\begin{array}{c}\text { mean } \\
\pm S D\end{array}$ & & $\mathbf{N}$ & $\begin{array}{c}\text { mean } \\
\pm \text { SD }\end{array}$ \\
\hline $\begin{array}{l}\text { Sphärisches } \\
\text { Äquivalent }\end{array}$ & 9 & $-0,3 \pm 2,1$ & 8 & $0,3 \pm 1,8$ & 0,49 & 6 & $\begin{array}{c}-0,3 \pm \\
2,3\end{array}$ & 28 & $\begin{array}{c}-0,2 \pm \\
2,2\end{array}$ & 0,92 & 34 & $\begin{array}{c}-0,2 \pm \\
2,2\end{array}$ \\
\hline
\end{tabular}




\subsection{Visus- und Kontrastvisusbestimmung}

Über das gesamte Patientenkollektiv gemittelt lag die durchschnittliche Punktzahl der Visusprüfung bei 79,8 von 100 möglichen Punkten \pm 1,5. Bei der Bestimmung des high-contrast-Visus ergab sich im Mittel eine Punktzahl von 47,6 \pm 9,2, die Auswertung der low-contrast-Visus-Untersuchung zeigte einen Durchschnitt von 38,7 Punkten \pm 11,3. Bei beiden Messungen war eine Maximalpunktzahl von 55 erreichbar.

In der Schwellenwert-Analyse (siehe Tabelle 10) war erkennbar, dass in allen drei gemessenen Kategorien in der Gruppe mit einer FAZ-Größe kleiner oder gleich 0,35 mm (G1) bessere Ergebnisse beobachtet wurden als in G2. Auch lagen die ermittelten Werte stets über dem Durchschnitt des Gesamtkollektivs. Besonders bei der Messung des low-contrast-Visus war ein deutlicher Unterschied zwischen den Ergebnissen zu verzeichnen $(p=0,17)$. Die Patienten mit wenig Ischämie (G1) erreichten rund sieben Punkte mehr als die Patienten, bei denen die Größe der FAZ über dem Normalwert von 0,35 $\mathrm{mm}^{2}$ lag (G2). Der Visus lag in $\mathrm{G} 1$ etwa vier Punkte oberhalb von Gesamtdurchschnitt und G2 ( $p=0,25)$, der high-contrast-Visus etwa drei Punkte $(p=0,38)$. In Bezug auf die zu erreichende Maximalpunktzahl von 55 Punkten in den Kontrastvisusuntersuchungen erreichten sowohl die Patienten aus G1 als auch G2 im Schnitt bessere Ergebnisse in der high-contrast-Messung.

Tabelle 10: Visus- und Kontrastvisusbestimmung [Schwellenwert-Analyse in Gruppen G1 und G2]

\begin{tabular}{|c|c|c|c|c|c|c|c|}
\hline & \multicolumn{2}{|c|}{$\begin{array}{c}\leq 0,35 \mathrm{~mm}^{2} \text { (G1) } \\
(\mathrm{N}=6)\end{array}$} & \multicolumn{2}{|c|}{$\begin{array}{c}>0,35 \mathrm{~mm}^{2}(\mathrm{G} 2) \\
(\mathrm{N}=30)\end{array}$} & \multicolumn{2}{|c|}{ Total $(\mathrm{N}=36)$} & \multirow{2}{*}{$\begin{array}{c}\text { t-test } \\
\text { p-value }\end{array}$} \\
\hline & $\mathbf{N}$ & mean \pm SD & $\mathbf{N}$ & mean \pm SD & $\mathbf{N}$ & mean \pm SD & \\
\hline ETDRS Visual Acuity Score & 6 & $83 \pm 7,2$ & 26 & $79,1 \pm 7,3$ & 32 & $79,8 \pm 1,5$ & 0,25 \\
\hline Kontrastvisus - high & 6 & $50,7 \pm 6,1$ & 27 & $47 \pm 9,7$ & 33 & $47,6 \pm 9,2$ & 0,38 \\
\hline Kontrastvisus - low & 6 & $44,5 \pm 6,7$ & 27 & $37,4 \pm 11,8$ & 33 & $38,7 \pm 11,3$ & 0,17 \\
\hline
\end{tabular}

Auch bei der Quartil-Analyse fiel auf, dass die Patienten aus der Extremgruppe Q1 mit den geringsten FAZ-Größen deutlich bessere Punktzahlen in den Visus-Untersuchungen aufwiesen als die Patienten aus Q3 (siehe Tabelle 11). Der größte Unterschied zwischen den zwei Gruppen fand sich auch hier in der low-contrast-Untersuchung mit einer Differenz von fast 10 Punkten $(p=0,14)$. Im Vergleich zwischen der Schwellenwert- und der Quartil-Analyse fiel auf, dass sich die Messwerte für die Sehschärfe und den high-contrast-Visus zwischen den Gruppen G1 und Q1 sowie G2 und 
Q3 nur wenig unterschieden. Bezogen auf die Sehschärfenmessung fand sich nicht nur die fast identische Differenz zwischen beiden festgelegten Gruppen, sondern auch kaum unterschiedliche absolute Punktzahlen. In der high-contrast-Untersuchung bestanden ebenfalls nur geringfügige Unterschiede zwischen den Gruppen im Vergleich der beiden Analysen. Lediglich in Q3 war ein um etwa drei Punkte geringeres Ergebnis zu verzeichnen als in G2.

Tabelle 11: Visus- und Kontrastvisusbestimmung [Quartil-Analyse in Gruppen Q1 und Q3]

\begin{tabular}{|c|c|c|c|c|c|}
\hline & \multicolumn{2}{|c|}{$\leq 0,45 \mathrm{~mm}^{2}(\mathrm{Q} 1)(\mathrm{N}=9)$} & \multicolumn{2}{|c|}{$\geq 1,16 \mathrm{~mm}^{2}(\mathrm{Q} 3)(\mathrm{N}=9)$} & \multirow{2}{*}{$\begin{array}{c}\text { t-test } \\
p \text {-value }\end{array}$} \\
\hline & $\mathbf{N}$ & mean \pm SD & $\mathbf{N}$ & mean \pm SD & \\
\hline ETDRS Visual Acuity Score & 9 & $83,4 \pm 5,8$ & 8 & $79,4 \pm 8,6$ & 0,28 \\
\hline Kontrastvisus - high & 9 & $51,9 \pm 5,2$ & 8 & $44 \pm 14,5$ & 0,18 \\
\hline Kontrastvisus - low & 9 & $43,9 \pm 5,6$ & 8 & $34 \pm 16,4$ & 0,14 \\
\hline
\end{tabular}




\subsection{Gesichtsfeldmessung}

Bei der absoluten Lichtempfindlichkeit (MS) lagen die Schwellenwerte über das gesamte Patientenkollektiv gemittelt bei etwa $27 \mathrm{~dB} \pm 4,8$ (siehe Tabelle 12). Sowohl in Q1 als auch in $\mathrm{G1}$, also den Gruppen mit einer avaskulären Fläche kleiner als $0,35 \mathrm{~mm}^{2}$, befanden sich die mittleren Schwellenwerte geringfügig oberhalb dieses Werts (siehe Tabelle 13). Bei den Patienten mit einer FAZ größer als 0,35 $\mathrm{mm}^{2}$ lag der durchschnittliche Wert für die Lichtempfindlichkeit etwas unterhalb des Mittelwerts. Auch die Messergebnisse des Defektwerts (MD) zeigten bessere Ergebnisse in den Gruppen Q1 und G1. Während der MD hier stets innerhalb der Toleranzbreite von $-2 \mathrm{~dB}$ bis $+2 \mathrm{~dB}$ lag, zeigten sich für die Gruppen Q3 und G2 mittlere Defektwerte außerhalb dieses Bereichs. Bei Betrachtung des Gesamtkollektivs war erkennbar, dass auch hier im Mittel der MD außerhalb des Toleranzbereichs lag. Die größte Abweichung fand sich bei Patienten, deren FAZ größer als $0,35 \mathrm{~mm}^{2}$ Fläche maß.

Tabelle 12: Gesichtsfeldmessung [Schwellenwert-Analyse in Gruppen G1 und G2, fett gedruckt Werte mit statistischer Signifikanz]

\begin{tabular}{|c|c|c|c|c|c|c|c|}
\hline & \multicolumn{2}{|c|}{$\leq 0,35 \mathrm{~mm}^{2}(\mathrm{G} 1)$} & \multicolumn{2}{|c|}{$>0,35 \mathrm{~mm}^{2}(\mathrm{G} 2)$} & \multicolumn{2}{|c|}{$(N=36)$} & t-test \\
\hline & $\mathbf{N}$ & mean \pm SD & $\mathbf{N}$ & mean \pm SD & $\mathbf{N}$ & mean \pm SD & p-value \\
\hline mean sensitivity $[\mathrm{dB}]$ & 6 & $28,7 \pm 1,4$ & 29 & $26,4 \pm 5,2$ & 35 & $26,8 \pm 4,8$ & 0,04 \\
\hline mean defect $[\mathrm{dB}]$ & 6 & $0,8 \pm 1,4$ & 29 & $2,8 \pm 4,5$ & 35 & $2,4 \pm 4,2$ & 0,07 \\
\hline
\end{tabular}

Tabelle 13: Gesichtsfeldmessung [Quartil-Analyse in Gruppen Q1 und Q3]

\begin{tabular}{|c|c|c|c|c|c|}
\hline & \multicolumn{2}{|c|}{$\leq 0,45 \mathrm{~mm}^{2}(\mathrm{Q} 1)(\mathrm{N}=9)$} & \multicolumn{2}{|c|}{$\geq 1,16 \mathrm{~mm}^{2}(\mathrm{Q} 3)(\mathrm{N}=9)$} & \multirow{2}{*}{$\begin{array}{c}\text { t-test } \\
\text { p-value }\end{array}$} \\
\hline & $\mathbf{N}$ & mean \pm SD & $\mathbf{N}$ & mean \pm SD & \\
\hline mean sensitivity $[\mathrm{dB}]$ & 8 & $28,7 \pm 1,3$ & 9 & $27,3 \pm 3,6$ & 0,32 \\
\hline
\end{tabular}

Bei der statistischen Aufarbeitung der Fangfragen-Befunde (siehe Abbildung 20) fiel auf, dass sich zwischen "falsch-positiven" und "falsch-negativen" Fangfragen ein gegengleicher Zusammenhang ergab. Während die Patienten der Gruppen mit einer kleinen FAZ (Säulen [2] und [4]) im Mittel höhere Anteile an "falsch-positiven" Fangfragen aufwiesen, als die Patienten der entsprechenden Vergleichsgruppen der jeweiligen Analyse (Säulen [3] und [5]), verhielt es sich bezüglich der Anteile der "falsch-negativen" Fangfragen umgekehrt. Hier zeigte sich, dass die Patienten mit einer 
größeren Ischämiefläche, in den Gruppen G2 und Q3 im Durchschnitt auf mehr Fangfragen reagierten als die Patientengruppen in G1 und Q1.

Generell fiel auf, dass der Anteil der "falsch-positiven" Fangfragen im Mittel deutlich über dem Anteil der "falsch-negativen" Fangfragen lag. Mit Blick auf das gesamte Patientenkollektiv war er etwa doppelt so hoch. Betrachtete man gesondert die Gruppen mit geringer FAZ-Größe (G1 und Q1) lagen die Anteile sogar zwischen fünf- und siebenfach höher. In den Kohorten G2 und Q3 drifteten die prozentualen Anteile deutlich weniger weit auseinander, was zum einen an weniger bejahten "falsch-positiven" Fangfragen lag und zum anderen durch den vergleichsweise größeren Anteil an "falsch-negativen" Fangfragen zustande kam.

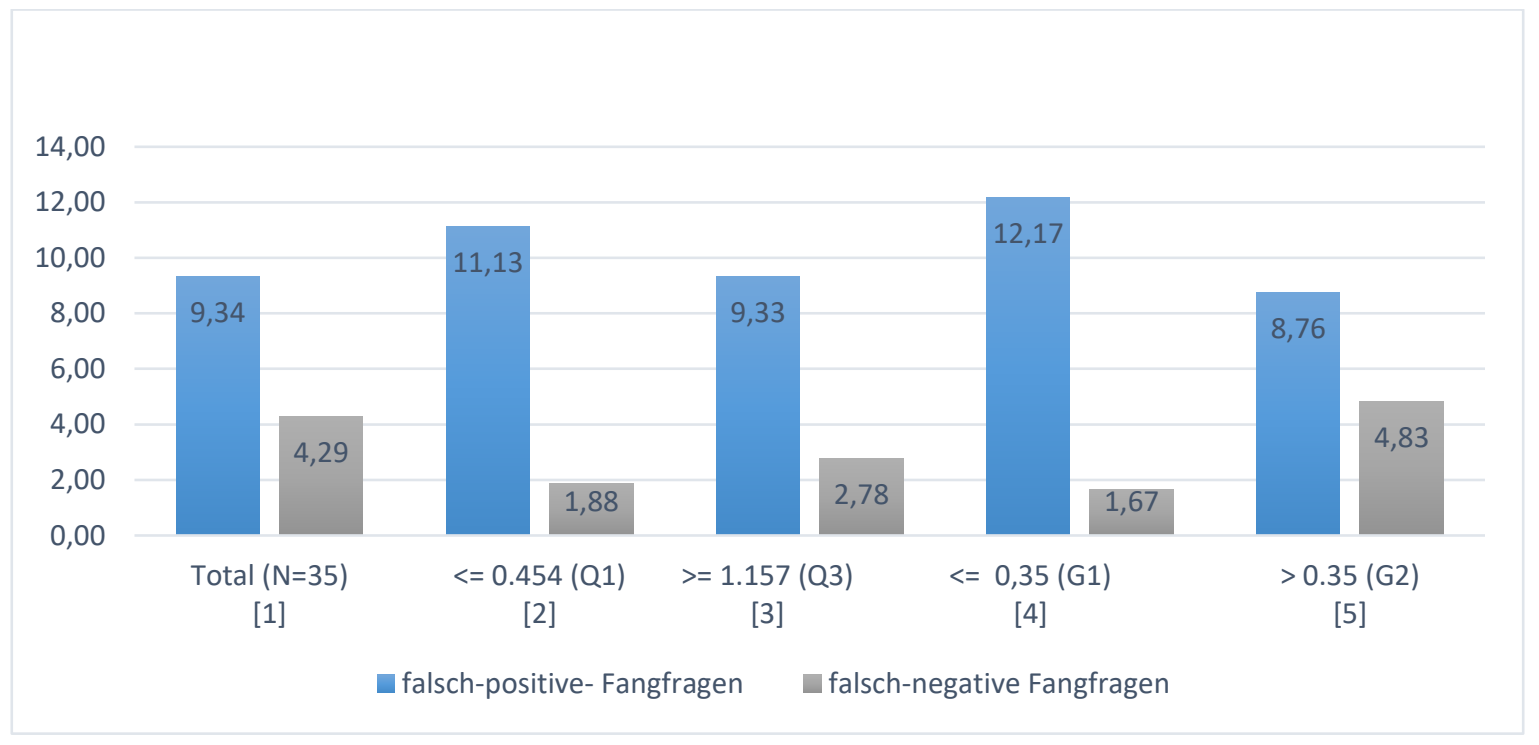

Abbildung 20: Mittelwerte der gewerteten Fangfragen in \% [Quartil-(Q1 und Q3) und SchwellenwertAnalyse (G1 und G2)] 


\subsection{Optische Kohärenztomografie (OCT)}

Die gemessenen Parameter in der OCT waren die Foveadicke (central subfield thickness, CSFT), die zentrale Foveadicke (central point thickness, CPT), das totale Makulavolumen (TMV) und das Foveavolumen (central subfield volume, CSFV). In der Studie wurden 34 OCT-Messungen ausgewertet und in Zusammenhang mit den gemessenen Ischämieflächen der Probanden in die vier Vergleichsgruppen eingeteilt. Tabelle 14 und Tabelle 15 zeigen die gemessenen Mittelwerte sowohl für die Schwellenwert- als auch für die Quartil-Analyse. Durchschnittlich lag die Dicke der gesamten Fovea bei 285,4 $\mu \mathrm{m} \pm 27,5$, bei einer im Mittel etwas geringeren Breite der zentralen Fovea von 250,6 $\mu \mathrm{m} \pm$ 39,5. Der Vergleich der Gruppen G1 und G2 zeigte, dass in den Dickenmessungen die Patienten mit einer Ischämiefläche kleiner als $0,35 \mathrm{~mm}^{2}$ (G1) auch geringere Werte bei den OCT-Indizes aufwiesen $(p(C P T)=0,75 ; p(C S F T)=0,74)$. Dasselbe galt für den Vergleich der gemessenen Volumina. Auch hier zeigte die Patientengruppe G1 geringere Messergebnisse als die Gruppe G2 (p(TMV) = 0,4; $($ CSFV $)=0,67)$. Im Durchschnitt erreichte das Gesamtkollektiv ein TMV von $8,7 \mathrm{~mm}^{3} \pm 0,7$ und ein CSFV von $0,2 \mathrm{~mm}^{3} \pm 0.02$

Tabelle 14: OCT-Parameter im Vergleich [Schwellenwert-Analyse in Gruppen G1 und G2]

\begin{tabular}{|c|c|c|c|c|c|c|c|}
\hline & \multicolumn{2}{|c|}{$\begin{array}{c}\leq 0,35 \mathrm{~mm}^{2}(\mathrm{G} 1) \\
(\mathrm{N}=6)\end{array}$} & \multicolumn{2}{|c|}{$\begin{array}{c}>0,35 \mathrm{~mm}^{2}(\mathrm{G} 2) \\
(\mathrm{N}=30)\end{array}$} & \multicolumn{2}{|c|}{ Total $(N=36)$} & \multirow{2}{*}{$\begin{array}{c}\text { t-test } \\
\begin{array}{c}p- \\
\text { value }\end{array}\end{array}$} \\
\hline & $\mathbf{N}$ & mean \pm SD & $\mathbf{N}$ & mean \pm SD & $\mathbf{N}$ & mean \pm SD & \\
\hline $\begin{array}{l}\text { center point thickness } \\
{[\mu \mathrm{m}]}\end{array}$ & 4 & $244,5 \pm 29,2$ & 30 & $251,4 \pm 41$ & 34 & $250,6 \pm 39,5$ & 0,75 \\
\hline $\begin{array}{l}\text { center subfield thickness } \\
{[\mu \mathrm{m}]}\end{array}$ & 4 & $281 \pm 33,2$ & 30 & $285,9 \pm 27,2$ & 34 & $285,4 \pm 27,5$ & 0,74 \\
\hline $\begin{array}{l}\text { total macular volume } \\
{\left[\mathrm{mm}^{3}\right]}\end{array}$ & 4 & $8,4 \pm 0,9$ & 30 & $8,7 \pm 0,7$ & 34 & $8,7 \pm 0,7$ & 0,4 \\
\hline $\begin{array}{l}\text { center subfield volume } \\
{\left[\mathrm{mm}^{3}\right]}\end{array}$ & 4 & $0,2 \pm 0,02$ & 30 & $0,2 \pm 0,02$ & 34 & $0,2 \pm 0,02$ & 0,67 \\
\hline
\end{tabular}

Der Vergleich der Extremgruppen in der Quartil-Analyse bestätigte die Tendenzen aus der Schwellenwert-Analyse. Auch hier lagen die Messergebnisse für die Gruppe mit der geringeren FAZ-Größe (Q1) deutlich unterhalb derer der Vergleichsgruppe Q3. Im Vergleich zur SchwellenwertAnalyse lagen hier die Ergebnisse der zwei Gruppen bezüglich CSFT, CPT und TMV noch weiter auseinander. In Gruppe Q3 fanden sich zudem für dieselben Parameter die größten Werte im Vergleich zwischen allen getesteten Gruppen. Das center subfield volume lag für die Gruppen mit 
geringerer FAZ-Größe in beiden Analysen (G1 und Q1), sowie für den Gesamtdurchschnitt und für die Gruppen $\mathrm{G} 2$ und Q3 bei $0,2 \mathrm{~mm}^{3} \pm 0,02$.

Tabelle 15: OCT-Parameter im Vergleich [Quartil-Analyse in Gruppen Q1 und Q3]

\begin{tabular}{|c|c|c|c|c|c|}
\hline & \multicolumn{2}{|c|}{$\leq 0,45 \mathrm{~mm}^{2}(\mathrm{Q} 1)(\mathrm{N}=9)$} & \multicolumn{2}{|c|}{$\leq 1,16 \mathrm{~mm}^{2}(\mathrm{Q} 3)(\mathrm{N}=9)$} & \multirow{2}{*}{$\frac{t \text {-test }}{\text { p-value }}$} \\
\hline & $\mathbf{N}$ & mean \pm SD & $\mathbf{N}$ & mean \pm SD & \\
\hline center point thickness $[\mu \mathrm{m}]$ & 7 & $247,9 \pm 29,6$ & 9 & $257,7 \pm 34$ & 0,55 \\
\hline center subfield thickness $[\mu \mathrm{m}]$ & 7 & $283,4 \pm 24,2$ & 9 & $294 \pm 25$ & 0,41 \\
\hline total macular volume $\left[\mathrm{mm}^{3}\right]$ & 7 & $8,5 \pm 0,7$ & 9 & $9,1 \pm 0,7$ & 0,12 \\
\hline center subfield volume $\left[\mathrm{mm}^{3}\right]$ & 7 & $0,2 \pm 0,02$ & 9 & $0,2 \pm 0,02$ & 0,31 \\
\hline
\end{tabular}

Zur Veranschaulichung dienen die Grafiken in Abbildung 21 und Abbildung 22. In der Balkendiagramm-Darstellung ergab sich für die Verteilung der Mittelwerte stets das gleiche Muster. Vom kleinsten bis zum größten Mittelwert staffelten sich die einzelnen Gruppen in der Folge G1 < Q1 < G2 < Q3 auf. Der Durchschnittswert des Gesamtkollektivs lag in der Mitte zwischen den Gruppen Q1 und G2. Die Patienten mit den vier geringsten FAZ-Größen wiesen in der Konsequenz die OCT-Indizes mit der kleinsten Abweichung zum Normalwert auf. Mit zunehmender Größe der Fovea nahm der Abstand zur Norm weiter zu. Ein Grund hierfür kann die Ausbildung eines Makulaödems mit zunehmender Größe der Ischämiefläche darstellen. Mit vermehrter ischämischer Schädigung der Netzhaut gehen auch Veränderungen im Sinne einer Flüssigkeitsansammlung im Bereich der Makula ausgelöst durch Ausschüttung von VEGF einher und werden in der OCT als Schichtdickenzunahme auffällig. 


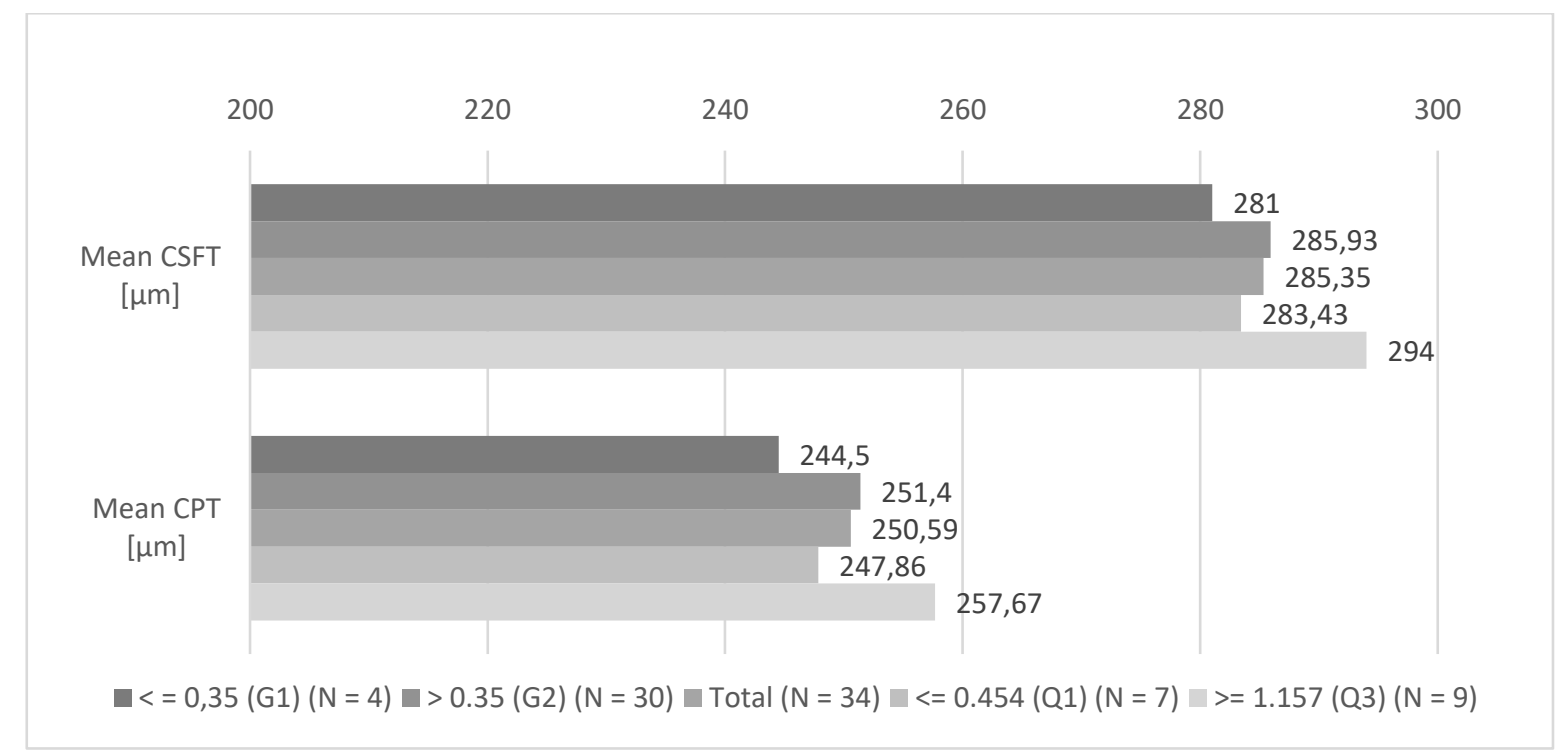

Abbildung 21: Analyse-übergreifende Darstellung von mean center subfield thickness [ $\mu \mathrm{m}]$ und mean center point thickness [ $\mu \mathrm{m}$ ], [Quartil- (Q1 und Q3) und Schwellenwert-Analyse (G1 und G2)]

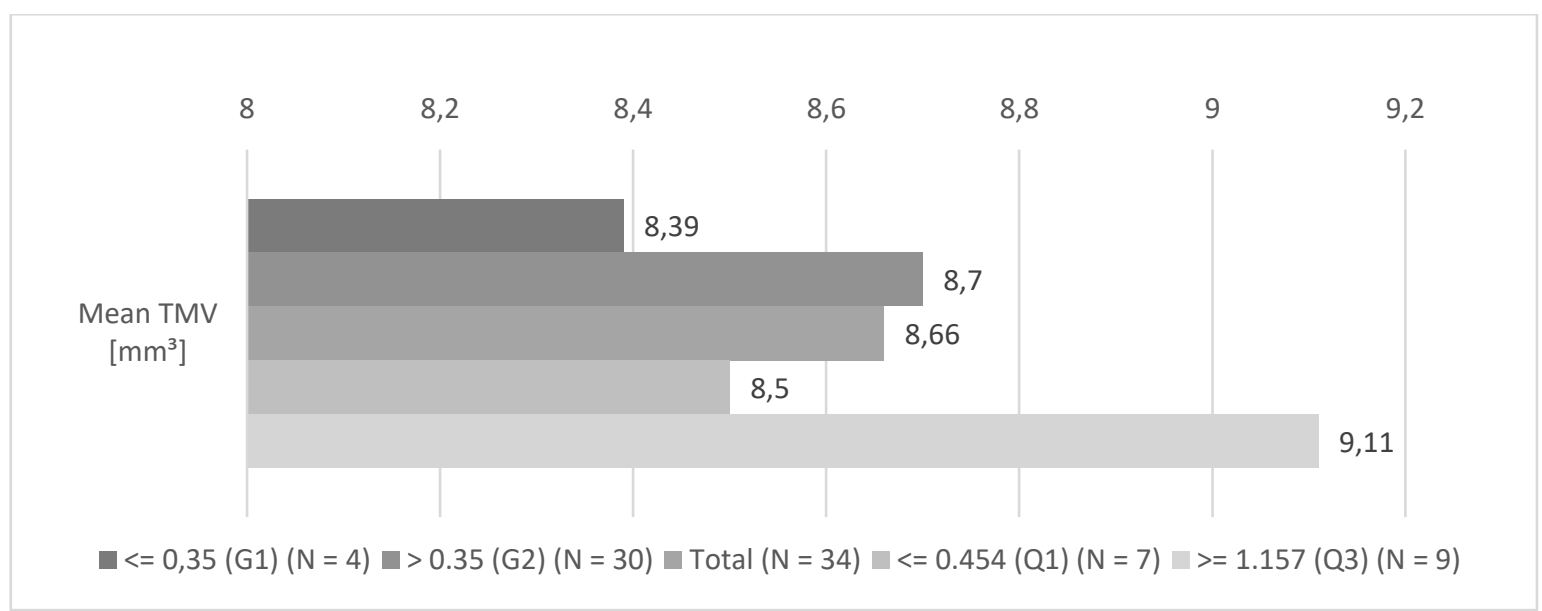

Abbildung 22: Analyse-übergreifende Darstellung des mean total macular volume [mm ${ }^{3}$, [Quartil- (Q1 und Q3) und Schwellenwert-Analyse (G1 und G2)] 


\subsection{Korrelationsanalysen}

Die im Folgenden aufgeführten Abbildungen zeigen jeweils auf der y-Achse aufgetragen die gemittelte Ischämiefläche aus der doppelt durchgeführten Ausmessung und auf der x-Achse den korrelierten Parameter aus Anamnese, Visus-, Kontrastvisus-, Gesichtsfeld- oder OCT-Untersuchung. Jede Grafik enthält eine Regressionsgerade, die den Zusammenhang zwischen den beiden Variablen verdeutlicht. Die Steigung dieser Graden entspricht dem Korrelationskoeffizienten nach Pearson.

\subsubsection{Ischämiefläche vs. demographische Daten}

Zur Korrelationsanalyse der demographischen Daten wurden als unabhängige Variablen (x-Achse) das Patientenalter, der $\mathrm{HbA}_{1 c}$ in \%, sowie die Dauer der Erkrankung und die Therapiedauer mit Insulin mit der Ischämiefläche als abhängige Variable (y-Achse) in Beziehung gesetzt. Die Tabelle 16 bietet eine Übersicht über die entsprechenden Ergebnisse des Korrelationskoeffizienten und Signifikanzwerte.

Die Abbildung 23 zeigt den Zusammenhang zwischen Patientenalter und Ischämiefläche $(p=0,23)$. Die Regressionsgerade wies eine Steigung von -0,21 auf. Es bestand somit ein negativer linearer Zusammenhang.

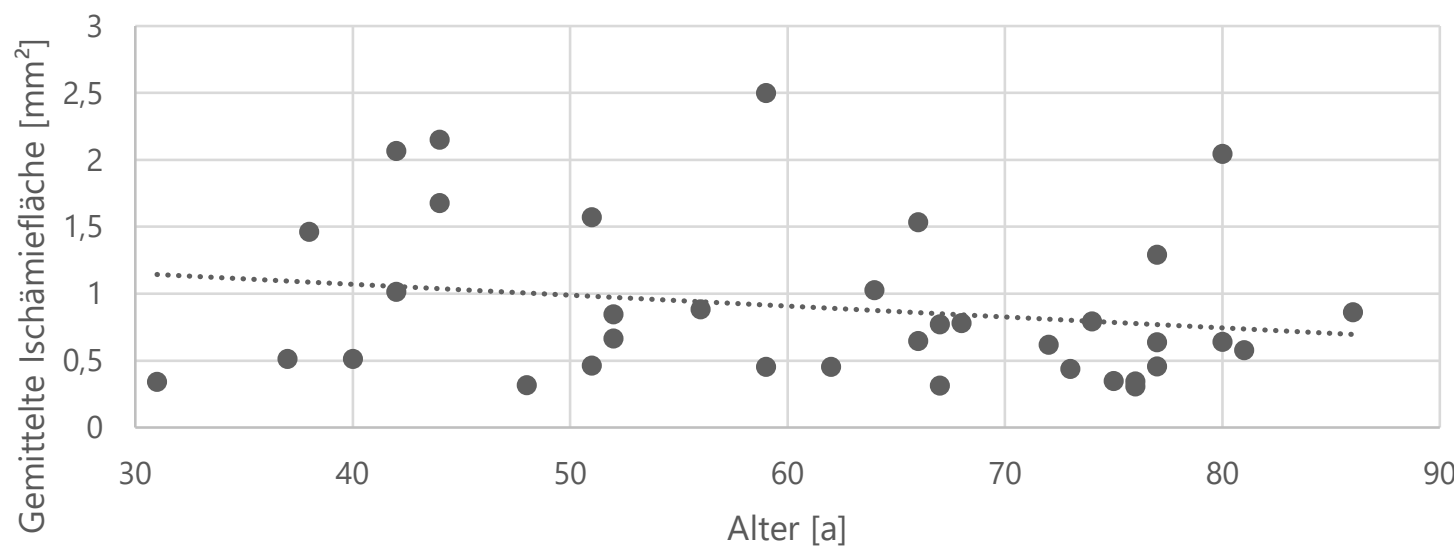

- Gemittelte Ischämiefläche vs. Alter $\quad$......... Regression

Abbildung 23: Gemittelte Ischämiefläche $\left[\mathrm{mm}^{2}\right]$ vs. Patientenalter [a] [Korrelationsanalyse; Korrelationskoeffizient $-0,21 ; p=0,23$ ] 
Die Abhängigkeit der beiden Variablen "Ischämiefläche" und " $\mathrm{HbA}_{1 c}$ " wurde in Abbildung 24 abgebildet. Der Verlauf der Regressionsgerade nähert sich fast einer horizontalen Linie, der Korrelationskoeffizient lag bei 0,02, der Signifikanzwert $p$ bei 0,92.

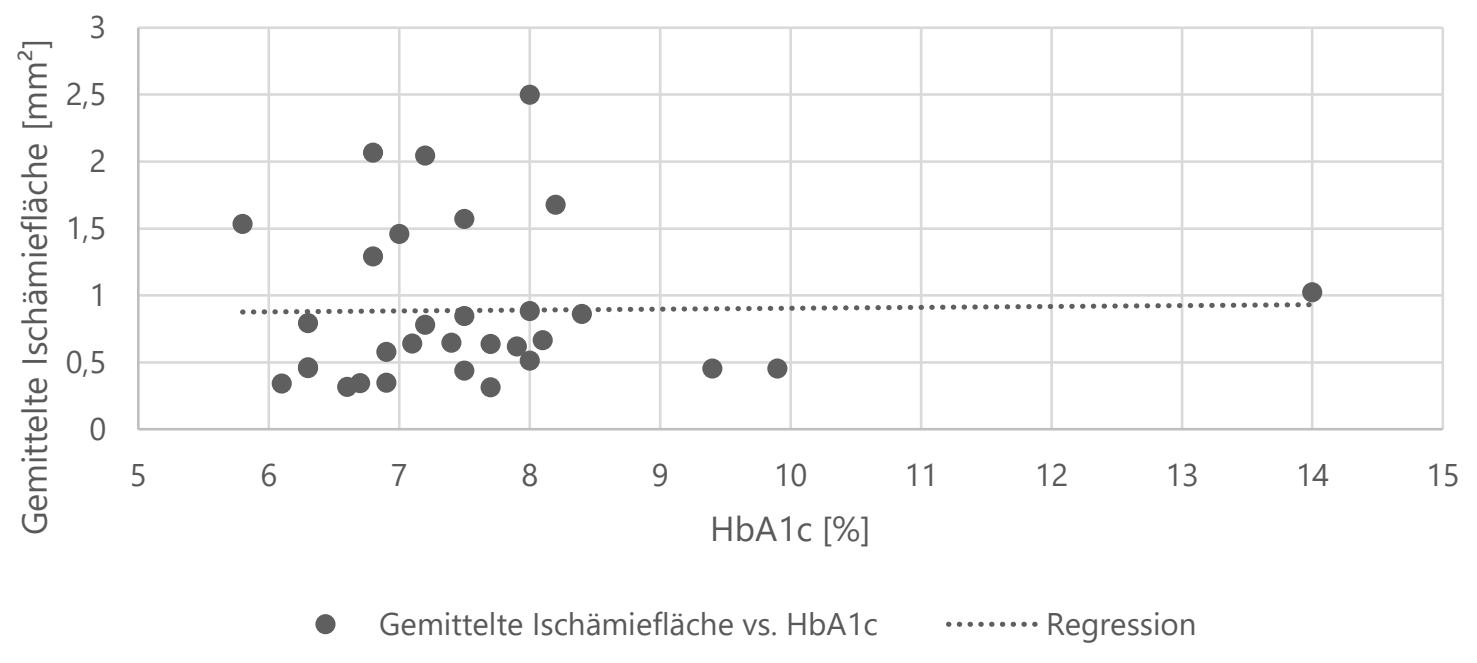

Abbildung 24: Gemittelte Ischämiefläche $\left[\mathrm{mm}^{2}\right]$ vs. HbA1c [\%] [Korrelationsanalyse; Korrelationskoeffizient 0,$02 ; p=0,92$ ]

In Abbildung 25 und Abbildung 26 wurden ebenfalls zwei unabhängige Variablen aufgetragen, die einem Zeitwert entsprechen. Es wurde hier sowohl die Ischämiefläche mit der Erkrankungsdauer als auch mit der Therapiedauer mit Insulin korreliert.

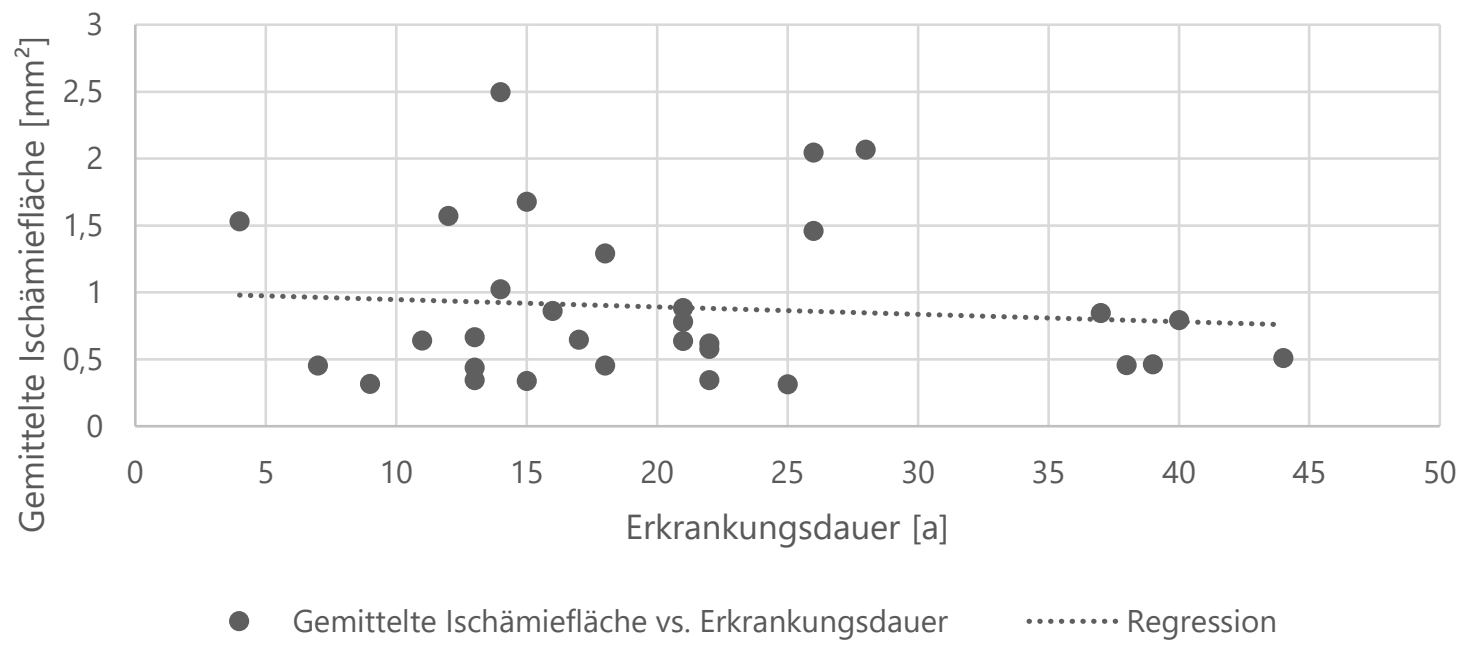

Abbildung 25: Gemittelte Ischämiefläche $\left[\mathrm{mm}^{2}\right]$ vs. Erkrankungsdauer [a] [Korrelationsanalyse; Korrelationskoeffizient $-0,1 ; p=0,61$ ] 
Während sich zwischen Fläche und Dauer des Diabetes ein negativer Zusammenhang erkennen ließ (Pearson - 0,$1 ; p=0,61$ ), zeigte die Korrelation zwischen Therapiedauer mit Insulin und Größe der Ischämiefläche ein gegensätzliches Bild (Pearson 0,05; $p=0,82$ ). Auch hier zeigte die Regressionsgerade jedoch einen annähernd horizontalen Verlauf mit niedriger Steigung.

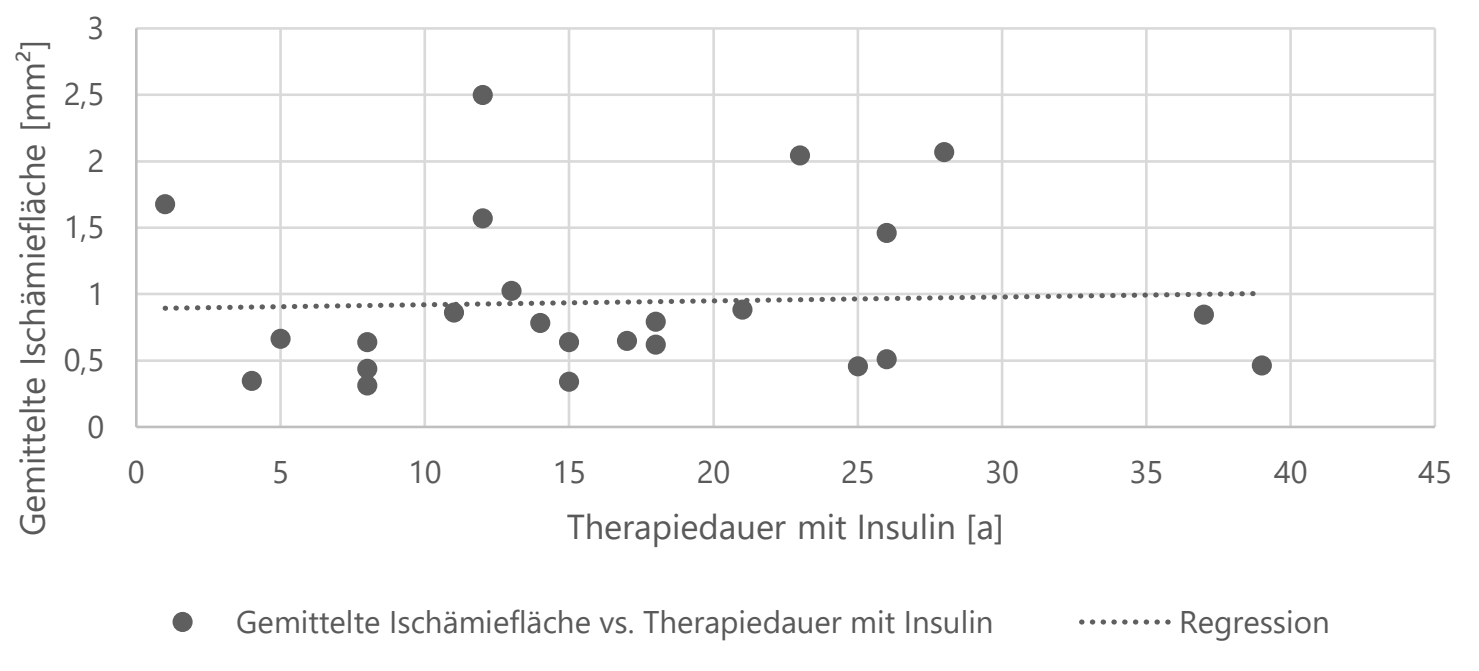

Abbildung 26: Gemittelte Ischämiefläche $\left[\mathrm{mm}^{2}\right]$ vs. Therapiedauer mit Insulin [a] [Korrelationsanalyse; Korrelationskoeffizient 0,05; $p=0,82$ ]

Für jeden demographischen Parameter wurde ebenfalls der Korrelationskoeffizient nach Spearman und der darauf bezogene $p$-Wert bestimmt (siehe Tabelle 16).

Tabelle 16: Übersicht Korrelationswerte Ischämiefläche vs. demografische Daten

\begin{tabular}{|l|c|c|c|c|}
\hline & $\begin{array}{c}\text { Korrelationskoeffizient } \\
\text { (Pearson) }\end{array}$ & $\begin{array}{c}\text { p-value(P): } \\
\text { H0:rho = }\end{array}$ & $\begin{array}{c}\text { Korrelationskoeffizient } \\
\text { (Spearman) }\end{array}$ & $\begin{array}{c}\text { p-value(S): } \\
\text { H0:rho = 0 }\end{array}$ \\
\hline $\begin{array}{l}\text { Ischämiefläche }\left[\mathrm{mm}^{2}\right] \\
\text { vs. Alter [a] }\end{array}$ & $-0,21$ & 0,23 & $-0,16$ & 0,35 \\
\hline $\begin{array}{l}\text { Ischämiefläche }\left[\mathrm{mm}^{2}\right] \\
\text { vs. HbA }[\text { [\%] }\end{array}$ & 0,02 & 0,92 & 0,15 & 0,4 \\
\hline $\begin{array}{l}\text { Ischämiefläche }\left[\mathrm{mm}^{2}\right] \\
\text { vs. Erkrankungsdauer } \\
\text { [a] }\end{array}$ & $-0,1$ & 0,61 & 0,03 & 0,88 \\
\hline $\begin{array}{l}\text { Ischämiefläche }\left[\mathrm{mm}^{2}\right] \\
\text { vs. Therapiedauer mit } \\
\text { Insulin [a] }\end{array}$ & 0,05 & 0,82 & 0,12 & 0,58 \\
\hline
\end{tabular}




\subsubsection{Ischämiefläche vs. Visus}

Zur Untersuchung der Sehkraft zählten sowohl der ETRDS-Visus als auch beide KontrastvisusUntersuchungen. Für alle drei Methoden gilt der Grundsatz, dass eine hohe Punktzahl einem besseren Ergebnis entspricht. In Abbildung 27, Abbildung 28 und Abbildung 29 sind die VisusUntersuchungen gegen die Ischämiefläche aufgetragen.

Für den ETDRS-Visustest ergab sich ein Korrelationskoeffizient von -0,17 bei einem Signifikanzwert von $p=0,34$ (siehe Tabelle 17). Die Regressionsgerade bildete dementsprechend eine negative Koinzidenz ab: bei kleinerer Ischämiefläche waren die Visuswerte höher.

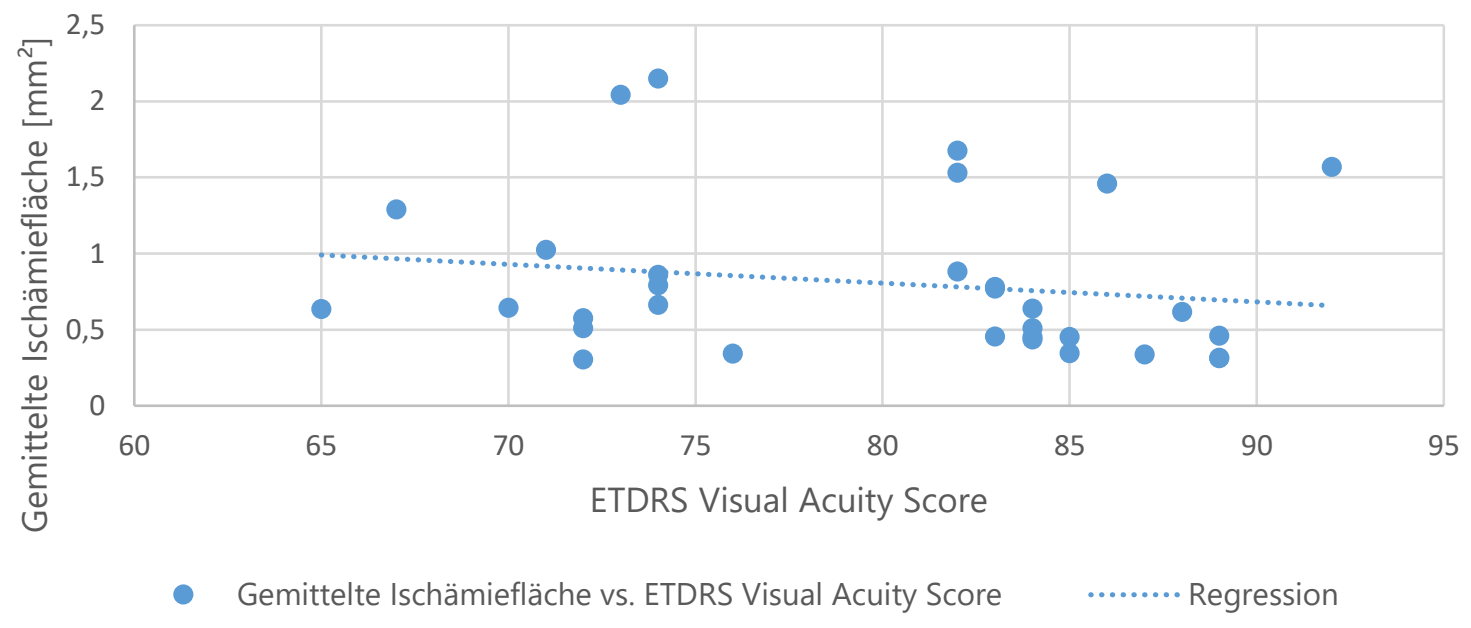

Abbildung 27: Gemittelte Ischämiefläche [ $\left.\mathrm{mm}^{2}\right]$ vs. ETDRS Visual Acuity Score [Korrelationsanalyse; Korrelationskoeffizient -0,17; $p=0,34$ ]

Gleiches galt für die Kontrastvisus-Untersuchungen. Die in Abbildung $\mathbf{2 8}$ dargestellte Analyse zum high-contrast-Visus zeigte ebenfalls eine Regressionsgerade mit einer negativen Steigung bei einem Korrelationskoeffizienten nach Pearson von $-0,38$ und einer Signifikanz von 0,03. In der Analyse nach Spearman zeigte sich ein geringfügig höherer $p$-Wert $(p=0,08)$. Das Ergebnis kann als signifikant bezeichnet werden.

In Abbildung 29 wurde die Korrelationsanalyse zum low-contrast-Visus veranschaulicht. Auch hier erkennt man einen negativen Zusammenhang (Pearson -0,41), der statistisch minimal größer ist als in der Untersuchung zum hohen Kontrast. Auch hierbei handelt es sich um ein signifikantes Ergebnis mit $p=0,02$ (siehe Tabelle 17) 


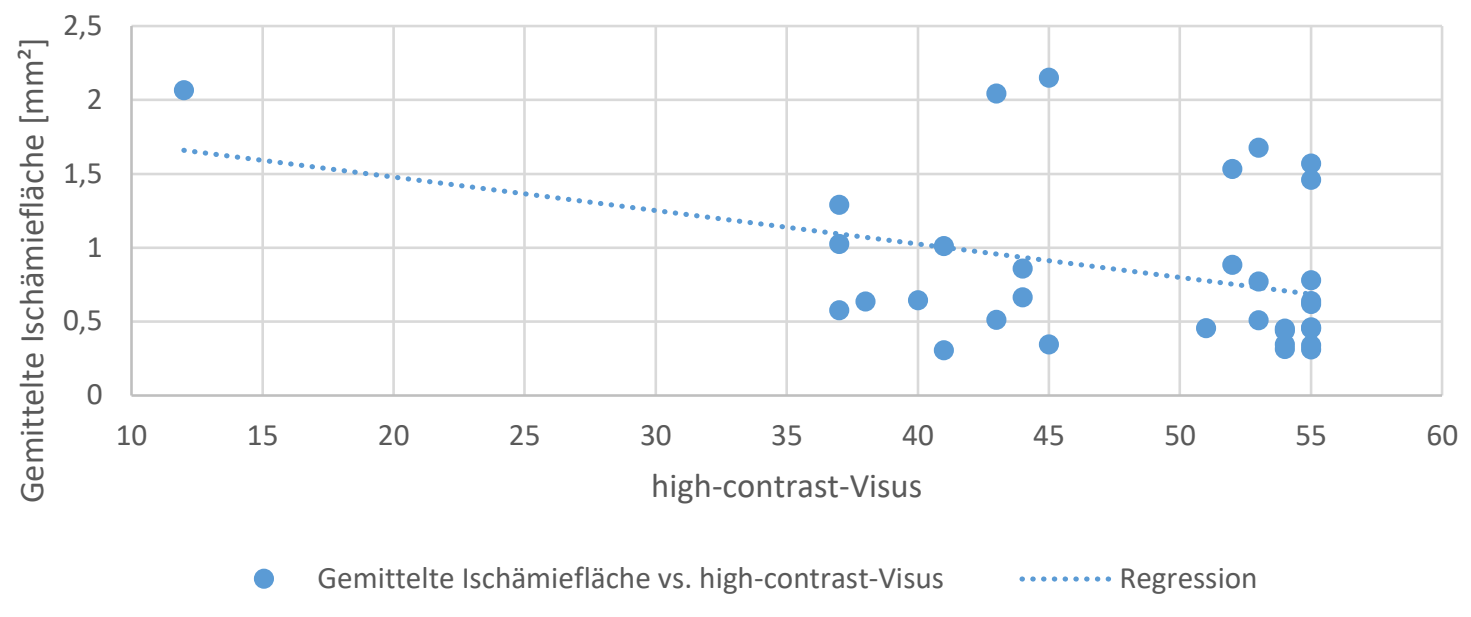

Abbildung 28: Gemittelte Ischämiefläche $\left[\mathrm{mm}^{2}\right]$ vs. high-contrast-Visus [Korrelationsanalyse, Korrelationskoeffizient $-0,38 ; p=0,03$ ]

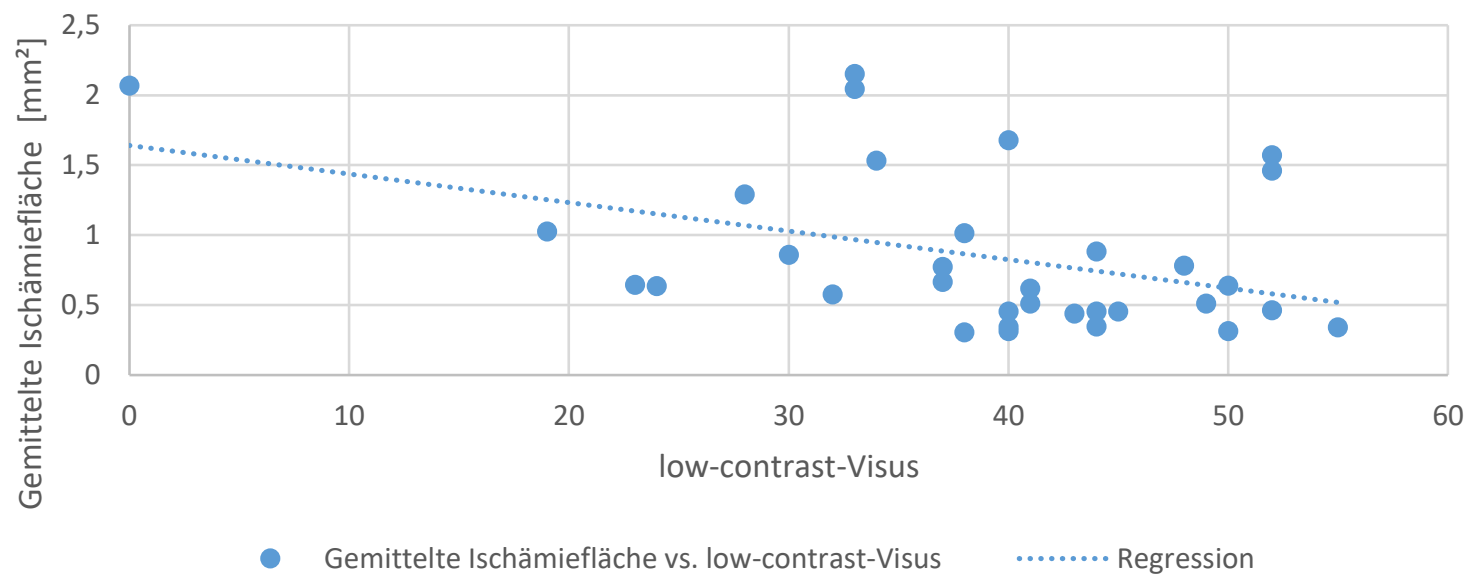

Abbildung 29: Gemittelte Ischämiefläche $\left[\mathrm{mm}^{2}\right]$ vs. low-contrast-Visus [Korrelationsanalyse; Korrelationskoeffizient $-0,41 ; p=0,02$ ]

Während die Korrelationskoeffizienten nach Pearson und Spearman (siehe Tabelle 17) annähernd vergleichbare Werte aufwiesen und stets auf einen negativen Zusammenhang hindeuteten, war erkennbar, dass der p-Wert als Maß für die Signifikanz in der Rangkorrelation für die Visus-Werte im Allgemeinen geringfügig bessere Werte aufwies. Vor allem für den ETRDS-Visus ergab sich ein deutlich besserer Wert bei $p=0,06$. 
Tabelle 17: Übersicht Korrelationswerte Ischämiefläche vs. Visus [fett gedruckt Werte mit statistischer Signifikanz]

\begin{tabular}{|l|c|c|c|c|}
\hline & $\begin{array}{c}\text { Korrelationskoeffizient } \\
\text { (Pearson) }\end{array}$ & $\begin{array}{c}\text { p-value(P): } \\
\text { H0:rho = }\end{array}$ & $\begin{array}{c}\text { Korrelationskoeffizie } \\
\text { nt } \\
\text { (Spearman) }\end{array}$ & $\begin{array}{c}\text { p-value(S): } \\
\text { H0:rho = }\end{array}$ \\
\hline $\begin{array}{l}\text { Ischämiefläche } \\
{\left[\mathrm{mm}^{2}\right] \text { vs. ETDRS }} \\
\text { Visual Acuity Score }\end{array}$ & $-0,17$ & 0,34 & $-0,33$ & 0,06 \\
\hline $\begin{array}{l}\text { Ischämiefläche } \\
{\left[\begin{array}{l}\left.\text { Imm }{ }^{2}\right] \text { vs. high- } \\
\text { contrast-Visus }\end{array}\right.}\end{array}$ & $-0,38$ & $\mathbf{0 , 0 3}$ & $-0,31$ & 0,08 \\
\hline $\begin{array}{l}\text { Ischämiefläche } \\
{\left[\begin{array}{l}\left.\text { Imm }{ }^{2}\right] \text { vs. low- } \\
\text { contrast-Visus }\end{array}\right.}\end{array}$ & $-0,41$ & $\mathbf{0 , 0 2}$ & $-0,39$ & $\mathbf{0 , 0 2}$ \\
\hline
\end{tabular}

\subsubsection{Ischämiefläche vs. perimetrische Daten}

Die Korrelationsanalyse der perimetrischen Daten umfasste die Ergebnisse der mean-sensitivityund der mean-defect-Erhebung. Dabei ist zu beachten, dass es sich bei der MS um einen absoluten Wert handelt, im Gegensatz zum MD, der bereits mit einem Vergleichswert korreliert wurde. Wie in Abbildung 30 und Abbildung 31 erkennbar, war der statistische Zusammenhang bei beiden perimetrischen Indizes durch geringe Werte des Korrelationskoeffizienten gekennzeichnet. Für die mean sensitivity ergab sich hier ein Wert von $-0,04$ bei $p=0,8$ (siehe Tabelle 18).

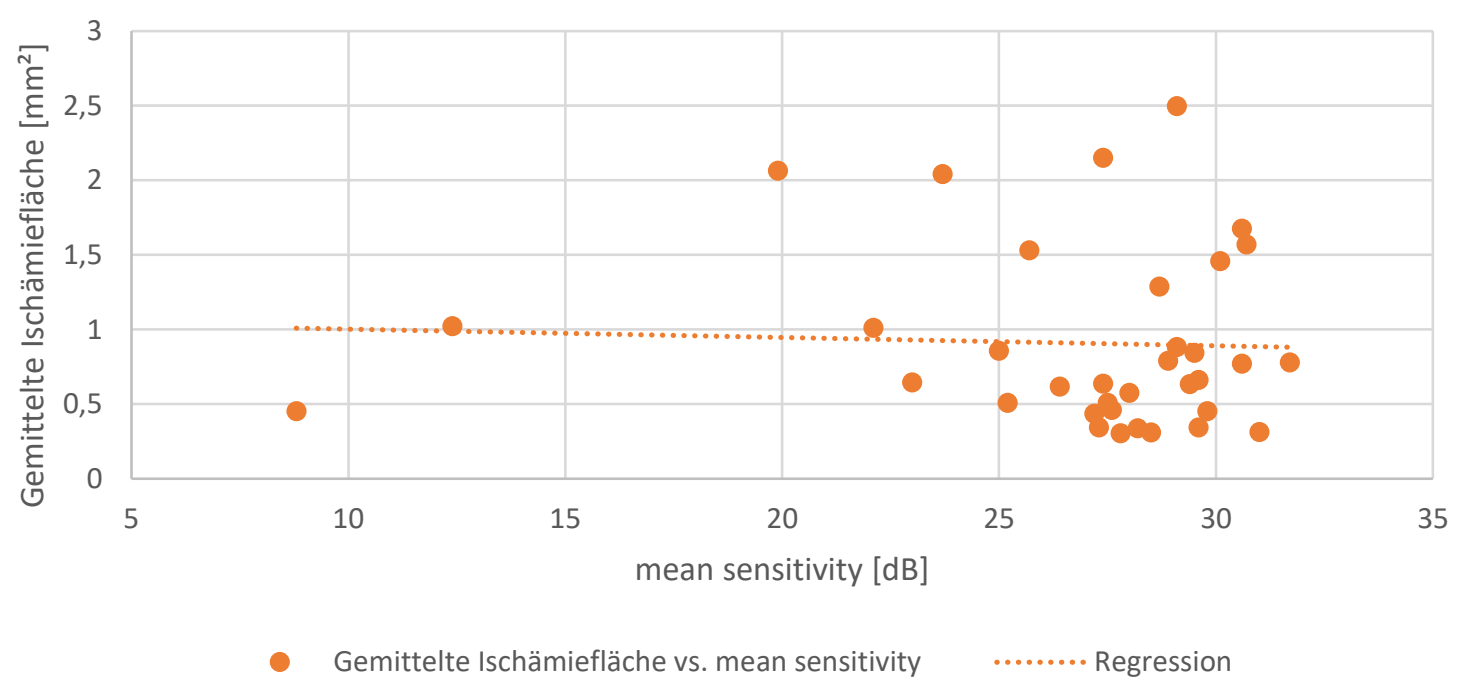

Abbildung 30: Gemittelte Ischämiefläche $\left[\mathrm{mm}^{2}\right]$ vs. mean sensitivity [dB] [Korrelationsanalyse; Korrelationskoeffizient $-0,04 ; p=0,8]$ 
Für die Analyse bezüglich des mean defect ergaben sich ähnliche Werte wie für die MS. Im Gegensatz zum vorherigen Wert lag jedoch ein geringfügig positiver Wert für den Korrelationskoeffizienten vor (Pearson 0,05). Bezüglich der Signifikanz ergibt sich $p=0,74$.

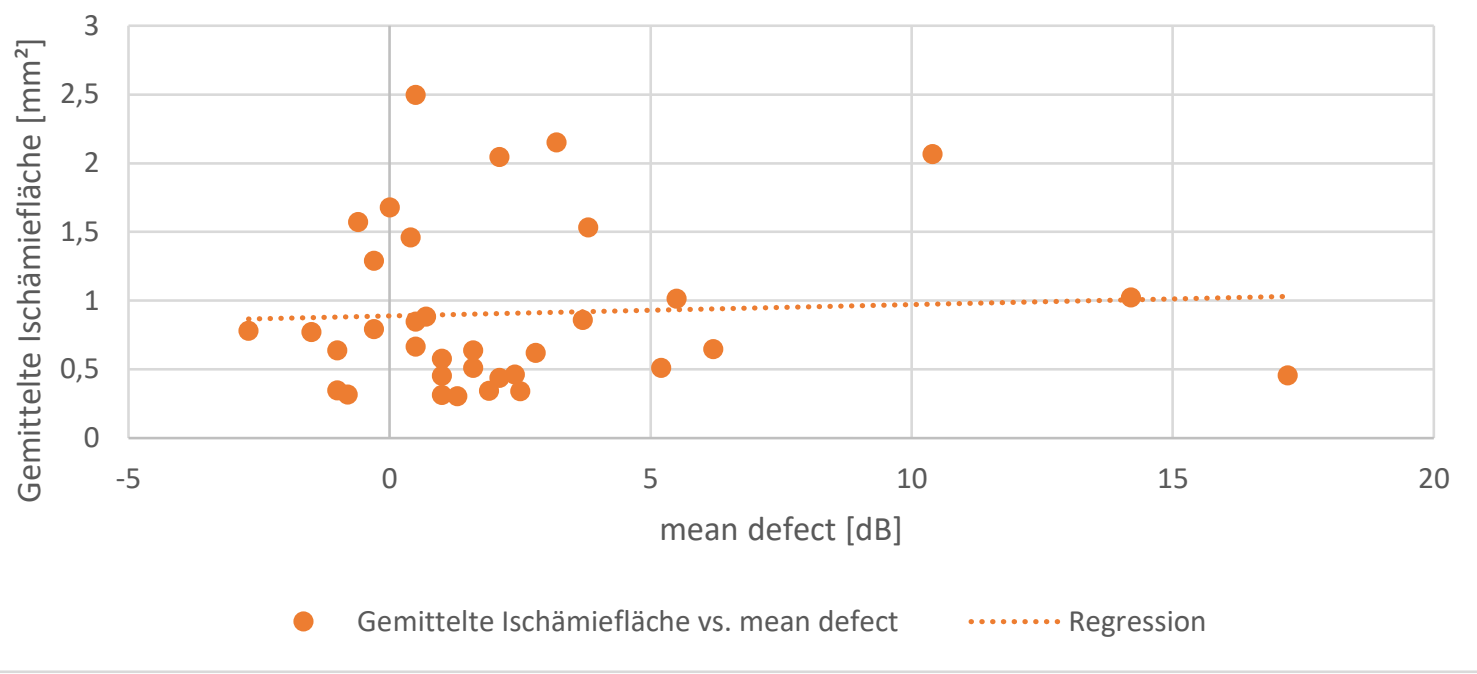

Abbildung 31: Gemittelte Ischämiefläche $\left[\mathrm{mm}^{2}\right]$ vs. mean defect [dB] [Korrelationsanalyse; Korrelationskoeffizient 0,05; $p=0,74]$

Die Rangkorrelation nach Spearman (siehe Tabelle 18) zeigte sowohl für die Koeffizienten als auch die Signifikanzwerte sehr ähnliche Ergebnisse.

Tabelle 18: Übersicht Korrelationswerte Ischämiefläche vs. perimetrische Daten

\begin{tabular}{|l|c|c|c|c|}
\hline & $\begin{array}{c}\text { Korrelationskoeffizient } \\
\text { (Pearson) }\end{array}$ & $\begin{array}{c}\text { p-value(P): } \\
\text { H0:rho }=\mathbf{0}\end{array}$ & $\begin{array}{c}\text { Korrelationskoeffizient } \\
\text { (Spearman) }\end{array}$ & $\begin{array}{c}\text { p-value(S): } \\
\text { H0:rho }=\mathbf{0}\end{array}$ \\
\hline $\begin{array}{l}\text { Ischämiefläche } \\
{\left[\mathrm{mm}^{2}\right] \text { vs. mean }} \\
\text { sensitivity [dB] }\end{array}$ & $-0,04$ & 0,8 & $-0,05$ & 0,77 \\
\hline $\begin{array}{l}\text { Ischämiefläche } \\
{\left[\begin{array}{l}\text { (mm }{ }^{2} \text { ] vs. mean } \\
\text { defect }[\mathrm{dB}]\end{array}\right.}\end{array}$ & 0,05 & 0,74 & 0,03 & 0,86 \\
\hline
\end{tabular}




\subsubsection{Ischämiefläche vs. OCT-Ergebnisse}

Im Rahmen der OCT-Untersuchung wurden für jeden Patienten vier verschiedene Parameter bestimmt. Bei zwei Werten handelte es sich um die Dickenmessung der retinalen Schicht an zwei verschiedenen Bereichen der Makula, die zwei weiteren Werte bezogen sich auf Angaben zu den Volumina von Makula und Fovea. Die entsprechenden Korrelationsanalysen in Bezug auf die Ischämiefläche werden in Abbildung 32 und Abbildung 33 gezeigt. Für beide Messungstypen ergaben sich Zusammenhänge mit positiver Steigung der jeweiligen Regressionsgeraden. Der Korrelationskoeffizient nach Pearson lag bei 0,21 (CPT) und 0,26 (CSFT).

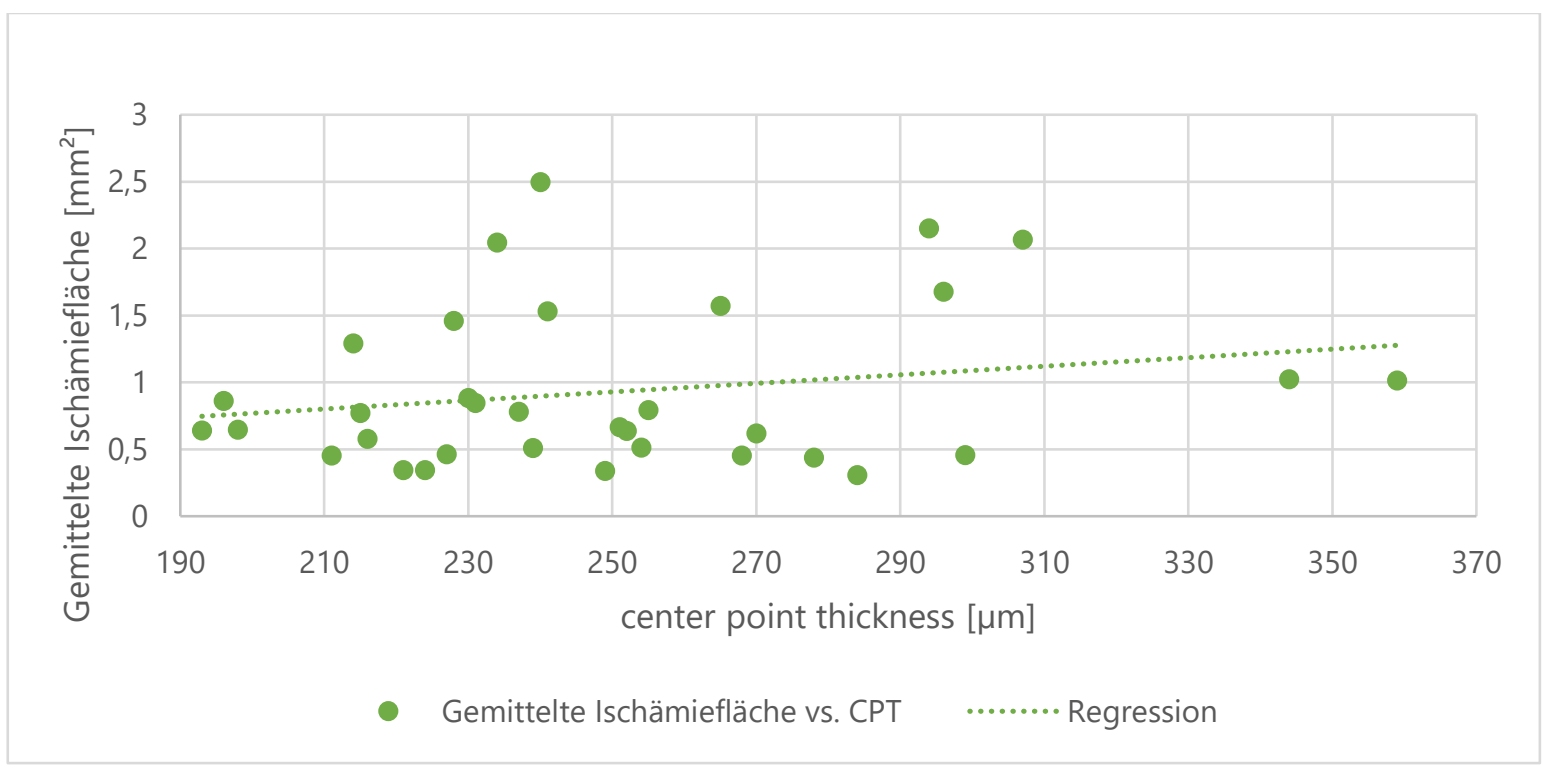

Abbildung 32: Gemittelte Ischämiefläche $\left[\mathrm{mm}^{2}\right]$ vs. center point thickness $[\mu \mathrm{m}]$ [Korrelationsanalyse; Korrelationskoeffizient 0,$21 ; p=0,23$ ] 


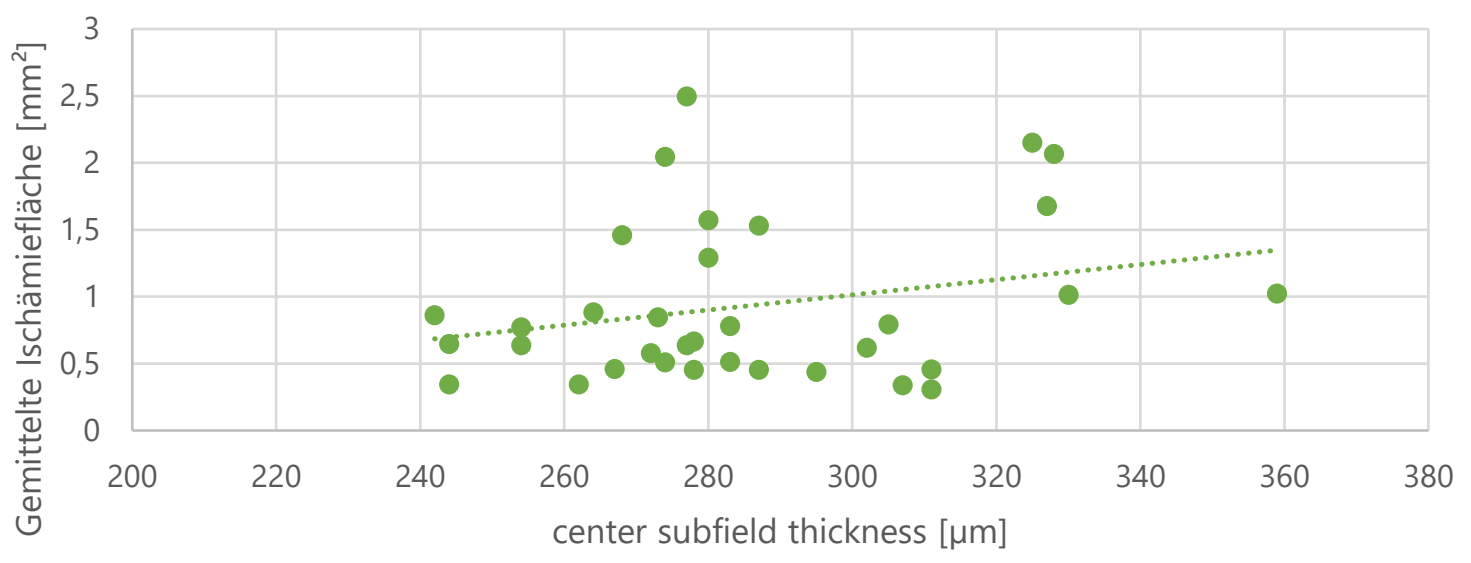

- Gemittelte Ischämiefläche vs. CSFT …..... Regression

Abbildung 33: Gemittelte Ischämiefläche $\left[\mathrm{mm}^{2}\right]$ vs. center subfield thickness [ $\left.\mu \mathrm{m}\right]$ [Korrelationsanalyse; Korrelationskoeffizient 0,26; $p=0,14]$

Der Signifikanzwert lag bei beiden Messungen bei $p>0,05$. Für die Analyse bezüglich des Zusammenhangs zwischen der center point thickness und der Ischämiefläche ergab sich ein $p=0,23$. Für die Dickenmessung im Bereich der Fovea (CSFT) galt $p=0,14$ (siehe Tabelle 19).

Weiterhin wurden Angaben zum Volumen von der gesamten Makula (TMV) und der Fovea (CSFV) erhoben. Die Grafik in Abbildung 34 zeigt die Korrelation bezogen auf das totale makuläre Volumen. Der Korrelationskoeffizient lag bei 0,35, der p-Wert bei 0,04. Auch hier bestand, wie bei den Kontrastvisus-Untersuchungen, ein statistisch signifikanter Zusammenhang zwischen dem erhobenen Wert und dem Ausmaß der Ischämie. Patienten mit einer ausgeprägteren Ischämie im Bereich der Macula lutea wiesen demnach auch größere Volumina in diesem Bereich auf. 


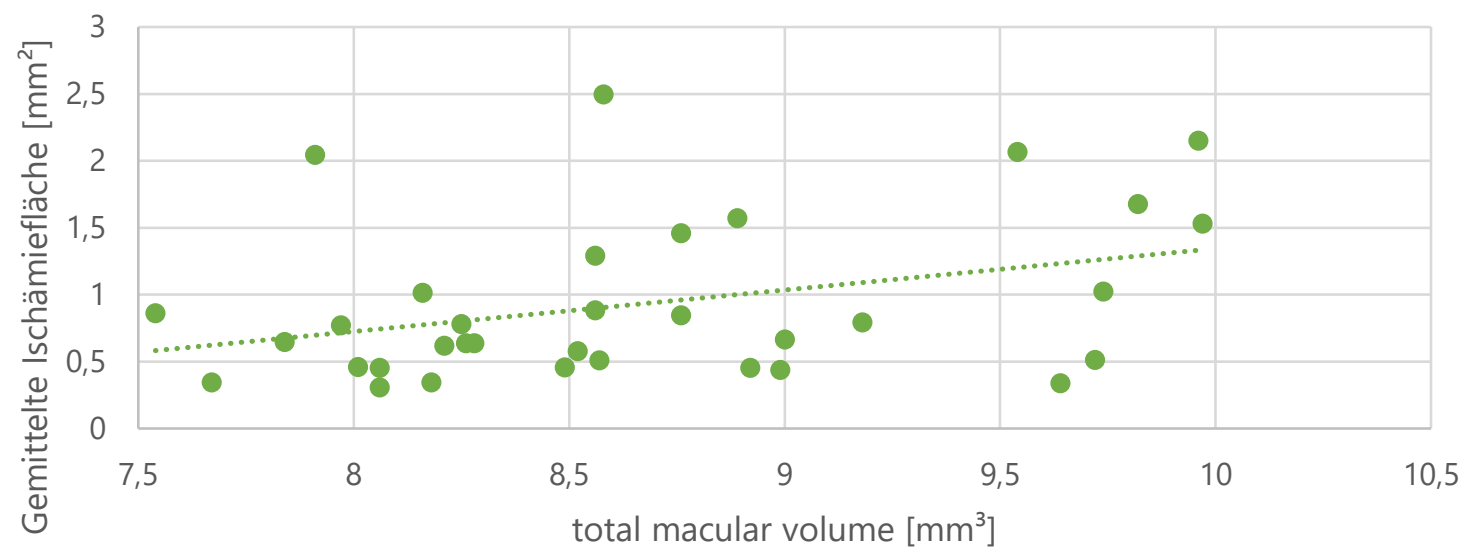

- Gemittelte Ischämiefläche vs. TMV $\quad$........ Regression

Abbildung 34: Gemittelte Ischämiefläche $\left[\mathrm{mm}^{2}\right]$ vs. total macular volume $\left[\mathrm{mm}^{3}\right]$ [Korrelationsanalyse; Korrelationskoeffizient 0,35; $p=0,04]$

Zusätzlich wurde das Volumen der Fovea gemessen, was dem center subfield volume entspricht. Auch bei dieser Messung ergab sich ein positiver Zusammenhang, der in der Steigung der Regressionsgeraden (Pearson 0,31) repräsentiert wird (siehe Abbildung 35). Für den Signifikanzwert wurde ein $p$ von 0,07 errechnet. Dies widerlegte knapp eine statistisch relevante Korrelation von Ischämiefläche und CSFV.

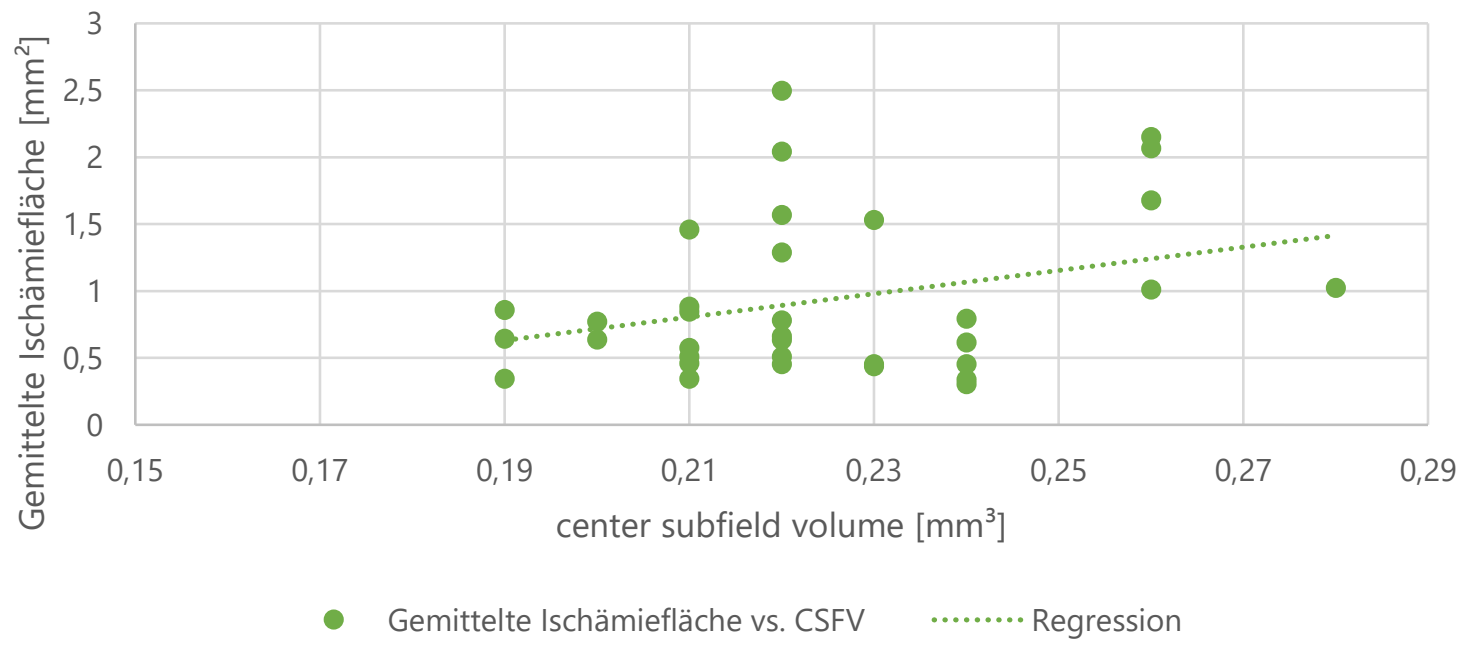

Abbildung 35: Gemittelte Ischämiefläche $\left[\mathrm{mm}^{2}\right]$ vs. center subfield volume $\left[\mathrm{mm}^{3}\right]$ [Korrelationsanalyse; Korrelationskoeffizient 0,31; $p=0,07]$ 
Im Gegensatz zur Korrelation zwischen Ischämiefläche und den in der perimetrischen Untersuchung erhobenen Daten) stellten sich die p-Werte nach Spearman im Vergleich zu Pearson in der Analyse bezogen auf die Messergebnisse aus der OCT, etwas schlechter dar. Für das totale makuläre Volumen änderte sich der $\mathrm{p}$-Wert soweit, dass die Korrelation das Signifikanz-Niveau verlässt $(p=0,06)$.

Tabelle 19: Übersicht Korrelationswerte Ischämiefläche vs. OCT-Ergebnisse [fett gedruckt Werte mit statistischer Signifikanz]

\begin{tabular}{|c|c|c|c|c|}
\hline & $\begin{array}{c}\text { Korrelationskoeffizient } \\
\text { (Pearson) }\end{array}$ & $\begin{array}{l}\text { p-value }(P): \\
\text { H0:rho }=0\end{array}$ & $\begin{array}{c}\text { Korrelationskoeffizient } \\
\text { (Spearman) }\end{array}$ & $\begin{array}{l}\text { p-value(S): } \\
\text { H0:rho = } 0\end{array}$ \\
\hline $\begin{array}{l}\text { Ischämiefläche } \\
{\left[\mathrm{mm}^{2}\right] \text { vs. center }} \\
\text { point thickness } \\
{[\mu \mathrm{m}]}\end{array}$ & 0,21 & 0,23 & 0,15 & 0,38 \\
\hline $\begin{array}{l}\text { Ischämiefläche } \\
{\left[\mathrm{mm}^{2}\right] \text { vs. center }} \\
\text { subfield thickness } \\
{[\mu \mathrm{m}]}\end{array}$ & 0,26 & 0,14 & 0,15 & 0,37 \\
\hline $\begin{array}{l}\text { Ischämiefläche } \\
{\left[\mathrm{mm}^{2}\right] \text { vs. total }} \\
\text { macular volume } \\
{\left[\mathrm{mm}^{3}\right]}\end{array}$ & 0,35 & 0,04 & 0,33 & 0,06 \\
\hline $\begin{array}{l}\text { Ischämiefläche } \\
{\left[\mathrm{mm}^{2}\right] \text { vs. center }} \\
\text { subfield volume } \\
{\left[\mathrm{mm}^{3}\right]}\end{array}$ & 0,31 & 0,07 & 0,2 & 0,25 \\
\hline
\end{tabular}




\section{Diskussion}

In dieser Arbeit sollte untersucht werden, ob es einen Zusammenhang zwischen dem morphologischen Kriterium ,makuläre Ischämie' einerseits und funktionellen Parametern andererseits gibt. Dazu wurde die Größe der FAZ den gemessenen funktionellen, aber auch weiteren morphologischen Parametern gegenübergestellt.

\subsection{Ergebnisinterpretation}

\subsubsection{Ausprägung der Ischämie}

Eine normal große FAZ weißt eine Größe von etwa 0,3-0,4 mm² auf (John et al. 2011; Chui et al. 2014; Carpineto et al. 2016). Im diabetischen Auge kann eine Vergrößerung dieses Gebietes auf $>0,4 \mathrm{~mm}^{2}$ auf eine makuläre Ischämie hindeuten.

In der untersuchten Stichprobe wies der Großteil der Probanden eine geringgradige bis mäßig ausgeprägte vergrößerte FAZ auf. Der Grund für diese Verteilung lag wahrscheinlich in der Selektion der rekrutierten Patienten. Durch die regelmäßige Untersuchung der Probanden durch spezialisierte Diabetologen der Diabetologischen Ambulanz der UMG sind die zu erwartenden diabetesbedingten Netzhautveränderungen vermutlich geringer ausgeprägt als bei Patienten, die sich aufgrund von Sehverschlechterung in der Augenklinik der UMG vorstellen.

\subsubsection{Zusammenhang zwischen Visus, Kontrastvisus und dem Grad der Ischämie}

Die Ergebnisse sowohl von Schwellenwert- und Quartilanalyse als auch der Korrelationsanalyse zeigten, dass Patienten mit gering ausgeprägter makulärer Ischämie höhere Punktzahlen in den Visusuntersuchungen, also eine höhere Sehkraft, aufwiesen. Dies galt gleichermaßen für ETDRSVisus und den Kontrastvisus. Zwischen der ETDRS-Visusmessung und fovealer Ischämiefläche wurde kein statistisch signifikanter Zusammenhang gefunden. Es ließ sich so nur eine Aussage zur Tendenz der Größe der Ischämiefläche nach Durchführung einer Visusuntersuchung machen. Statistisch relevant zeigten sich dagegen die Ergebnisse der Kontrastvisus-Korrelationsanalyse. Sowohl für den high-contrast- als auch für den low-contrast-Visus konnte ein Zusammenhang nachgewiesen werden. Bei Vorliegen einer diabetischen Retinopathie bestand also ein Abhängigkeitsverhältnis 
zwischen dem Ausmaß der Erkrankung und der Einschränkung der Kontrastsensitivität. Hierbei galt: je größer die Ischämiefläche, desto schlechter das Kontrastsehen.

Die aktuelle Studienlage unterstützt die Messergebnisse dieser Studie. Es ist bekannt, dass besonders in früheren Stadien einer Diabetes-Erkrankung Veränderungen der Kontrastsehschärfe zu verzeichnen sind (Sun und Zhang 2012). Dabei konnte nachgewiesen werden, dass auch die Zunahme der Ischämiefläche Einfluss auf das Kontrastsehen hat. Da auch andere typische Augenerkrankungen einen nachteiligen Einfluss auf das Kontrastsehen haben, haben wir diese ausgeschlossen. Dazu gehören vor allem eine relevante Linsentrübung (Katarakt), das Glaukom und Erkrankungen des Nervus opticus (Bach et al. 2008).

\subsubsection{Zusammenhang zwischen den Parametern der Perimetrie und dem Ischämiegrad}

Je größer die foveale Ischämie, desto größer zeigen sich die zentralen relativen Gesichtsfeldausfälle (relative Skotome) im Vergleich zur gesunden Kohorte. Die Ergebnisse der Schwellenwert-Analyse waren für die mean sensitivity statistisch signifikant. Für die Patienten mit vergrößerten fovealen avaskulären Zonen zeigten sich eine geringere mean sensitivity und höhere Defektwerte. Für die mean sensitivity als auch für den mean defect ließen sich im Rahmen der Korrelationsanalysen beide Zusammenhänge gleichermaßen nachweisen.

Der mean defect galt als wichtigster Parameter dieser Untersuchung. Im Gegensatz zur individuell sehr unterschiedlichen mean sensitivity bietet er ein objektives Maß - sowohl bezogen auf den getesteten Prüfort als auch auf das Alter des Patienten - und unterliegt deshalb weniger Schwankungen. Trotzdem fanden wir nur bei der mean sensitivity statisch signifikante Ergebnisse. Die Annahme, dass bei Patienten mit vermehrter ischämischer Schädigung (G2 und Q3) eine Vergrößerung der Gesichtsfeldausfälle vorliegt, konnte bestätigt werden. Neben den ausgewerteten Perimetrieparametern wurden außerdem die Angaben der Patienten bezogen auf die eingestreuten Fangfragen analysiert. Hierbei zeigte sich, dass die Probanden mit geringeren ischämischen Schäden höhere prozentuale Anteile bei den falsch-negativen Fangfragen aufwiesen (G1 und Q1), während es sich in Bezug auf die falsch-positiven Fangfragen umgekehrt verhielt. Hier beobachteten wir, dass besonders Patienten mit größeren ischämischen Bereichen die Fangfragen bejahten (G2 und Q3). Dies deutet daraufhin, dass Patienten mit geringerer Lichtempfindlichkeit und Sehkraft dazu tendieren, auf die Frage nach der Sichtbarkeit eines Lichtpunktes positiv zu antworten. Dies geschieht auch dann, wenn kein Lichtreiz präsentiert wurde, um ihre bestehende Sehschwäche zu kompensieren. 


\subsubsection{Zusammenhang zwischen der Netzhautdicke (OCT) und dem Grad der Ischämie}

Die Messergebnisse der OCT-Untersuchung zeigten für Patienten aus der Gruppe G1 (FAZ <=0,35 $\mathrm{mm}^{2}$ ) für die drei Parameter CSFT, CPT und TMV die geringsten, für Patienten aus der Gruppe Q3 (FAZ >= 1,16 $\mathrm{mm}^{2}$ ) die höchsten Werte. Die Korrelationsanalysen zeigten für alle vier Parameter positive Zusammenhänge bezogen auf die Größe der Ischämiefläche. Das heißt mit zunehmender Ischämiefläche nehmen sowohl die Netzhautdicke als auch Volumen an unterschiedlichen Punkten der Netzhaut zu. Im Vergleich zwischen den in der Literatur angegebenen Normwerten und den Studienergebnissen zeigte sich, dass in dieser Studie in Bezug auf Volumen und Dicke größere Durchschnittwerte gemessen wurden (vgl. Tabelle 20)

Tabelle 20: Vergleich Normwerte vs. Studienergebnisse

\begin{tabular}{|l|c|c|c|c|}
\hline & $\begin{array}{c}\text { mean CSFT } \\
{[\mu \mathrm{m}]}\end{array}$ & $\begin{array}{c}\text { mean CPT } \\
{[\mu \mathrm{m}]}\end{array}$ & $\begin{array}{c}\text { mean TMV } \\
{\left[\mathbf{m m}^{3}\right]}\end{array}$ & $\begin{array}{c}\text { mean CSFV } \\
{\left[\mathbf{m m}^{3}\right]}\end{array}$ \\
\hline Gesunde Probanden (Bressler et al. 2008) & $174-201$ & $144-211$ & $8,49-10,1$ & - \\
\hline Patienten mit Diabetes mellitus und/oder DR \\
(Bressler et al. 2008) & $179-256$ & $158-244$ & - \\
\hline$<=0,35 \mathrm{~mm}^{2}(\mathrm{G} 1)(\mathrm{N}=4)$ & & 281 & 8,39 & 0,22 \\
\hline$>0,35 \mathrm{~mm}^{2}(\mathrm{G} 2)(\mathrm{N}=30)$ & 285,93 & 251,4 & 8,7 & 0,23 \\
\hline Total $(\mathrm{N}=34)$ & 285,35 & 250,59 & 8,66 & 0,22 \\
\hline$<=0,45 \mathrm{~mm}^{2}(\mathrm{Q} 1)(\mathrm{N}=7)$ & 283,43 & 247,86 & 8,5 & 0,22 \\
\hline$>=1,16 \mathrm{~mm}^{2}(\mathrm{Q} 3)(\mathrm{N}=9)$ & 294 & 257,67 & 9,11 & 0,23 \\
\hline
\end{tabular}

Die Durchschnittswerte für die von einem OCT-Gerät gemessene Foveadicke unterscheiden sich deutlich bezüglich der Lokalisation und Untersuchungstechnik. Für die Dicke am center point (^ zentrale Foveadicke) gelten beispielsweise geräteabhängig Normwerte von 146 - $182 \mu \mathrm{m}$, während die Dicke im center subfield um den center point herum zwischen 170 und $212 \mu \mathrm{m}$ betragen kann (Hee et al. 1998; Massin et al. 2002; Chan et al. 2006; Koleva-Georgieva und Sivkova 2010). Auch bezüglich der Volumina lassen sich Aussagen über Durchschnittswerte treffen. Das Gesamtvolumen der Makula (total macular volume, TMV) beträgt in gesunden Augen im Mittel etwa zwischen $8,49 \mathrm{~mm}^{3}$ und $10,1 \mathrm{~mm}^{3}$ (Liu et al. 2011; Pokharel et al. 2016). Für das Foveavolumen 
(central subfield volume, CSFV) wurden Mittelwerte um etwa $0,2 \mathrm{~mm}^{3}$ beschrieben (Diaz et al. 2012; Pokharel et al. 2016).

Bei Patienten mit Diabetes mellitus finden sich auch höhere Durchschnittswerte für die retinale Dicke als bei gesunden Patienten, auch wenn sich keine Merkmale einer diabetischen Retinopathie finden lassen. Je nach Form der retinalen Schädigung ergeben sich unterschiedliche Durchschnittswerte bezüglich der Netzhautdicke an verschiedenen Lokalisationen (vgl. Tabelle 21).

Tabelle 21: Übersicht retinale Schichtdicken bei Patienten mit Diabetes mellitus (Bressler et al. 2008)

\begin{tabular}{|l|c|c|c|}
\hline \multicolumn{1}{|c|}{ Region } & Diabetes ohne DR & NPDR & PDR \\
\hline center point [in $\mu \mathrm{m}$ ] & 158 & 244 & 236 \\
\hline center subfield [in $\mu \mathrm{m}$ ] & 179 & 256 & 254 \\
\hline inner subfield [in $\mu \mathrm{m}$ ], gemittelt über 4 Quadranten & 256,25 & 292,75 & 299,75 \\
\hline outer subfield [in $\mu \mathrm{m}$ ], gemittelt über 4 Quadranten & 235 & 262,25 & 275 \\
\hline
\end{tabular}

Einige Studien zeigen jedoch auch, dass es bei fortgeschrittenen Erkrankungsverläufen auch zu einer atrophiebedingten Verdünnung der Netzhaut kommen kann. Dabei handelt es sich aber um Spätstadien der Erkrankung. Liegt eine retinale Ischämie vor, kommt es vor allem im Bereich der Ganglienzellschicht innerhalb der Netzhaut zum Abbau von Nervenzellgewebe und somit zur Verschmälerung dieses Bereichs (Byeon et al. 2009; van Dijk et al. 2010; Choi et al. 2017). Bereits 1998 konnte für Patienten mit Diabetes mellitus durchschnittliche Messwerte bezüglich der retinalen Dicke für jeden Bereich des Messradius abhängig vom Krankheitsprofil der Patienten erhoben werden (Hee et al. 1998). Im Rahmen verschiedener Studien mit neueren OCT-Geräten ergaben sich Durchschnittswerte für den CP von $144-211 \mu \mathrm{m}$ und das CSF von $174-201 \mu \mathrm{m}$ (Lattanzio et al. 2002; Massin et al. 2002; Bressler et al. 2008) für Patienten mit Diabetes und/oder minimaler diabetischer Retinopathie. Die Zunahme der retinalen Dicke im untersuchten Patientenkollektiv ist durch das vermehrte Auftreten eines zarten diabetischen Makulaödemen zu erklären. Das Makulaödem tritt häufig im Rahmen einer diabetischen Retinopathie auf und ist der häufigste Grund für den Verlust von Sehkraft bei Patienten mit Diabetes mellitus (Patelli et al. 2014). Ein klinisch signifikantes Makulaödem ist in der OCT nachweisbar und im Rahmen der Studie als Ausschlusskriterium definiert. Die Problematik in Bezug auf die Dicken- und Volumina-Messung liegt im Bereich der subklinischen Ödeme, die unterhalb der Nachweisgrenze des OCT-Geräts liegen. Im Rahmen der Diabetic Retinopathy Clinical Research Network Studien wurde als Schwellenwert für ein diabetisches Makulaödem eine retinale Dicke von über $250 \mu \mathrm{m}$ festgelegt (Diabetic Retinopathy Clinical Research Network 2009). Frühe ödematöse Veränderungen lassen 
sich bereits ab einer Dicke von etwa $240 \mu \mathrm{m}$ erkennen (Koleva-Georgieva und Sivkova 2010). Geringe Schwellungen der Retina durch Flüssigkeitseinlagerungen können jedoch unterhalb der Nachweisgrenze des OCT-Gerätes nicht gemessen und dem Untersucher angezeigt werden.

\subsubsection{Zusammenhang zwischen Alter, Diabetes-Typ und Grad der Ischämie}

Die in der aktuellen Studie erhobenen demografischen Daten, wie z.B. das Durchschnittsalter und die Verteilung der Diabetes-Typen, zeigen eine ähnliche Verteilung in der Gesamtbevölkerung (Heidemann et al. 2013), wodurch sich belegen lässt, dass die vorliegende Patientendaten eine repräsentative Gruppe darstellt. Die untersuchten Probanden dieser Studie waren im Durchschnitt etwa 61 Jahre alt. In der Quartil- und Schwellenwertanalyse zeigte sich, dass Probanden mit einer größeren Ischämiefläche ein niedrigeres Durchschnittsalter aufwiesen als solche mit weniger ausgedehnteren zentralen Ischämien. Diese Tendenz zeigte sich auch in der Auswertung der Daten innerhalb der Korrelationsanalyse. Dort ließ sich eine negative Korrelation nachweisen, die jedoch statistisch nicht signifikant war. Aufgrund des verhältnismäßig großen Anteils an Probanden mit Typ-1 Diabetes aus der Diabetes-Ambulanz lag in dem Anteil von Patienten mit einem Diabetes mellitus Typ-2 bei etwa 67\%, während in der Bevölkerung etwa 90\% der an Diabetes erkrankten Personen an einem Diabetes mellitus Typ-2 leiden. Diese Verteilung ist auf die besondere Patientenrekrutierung zurückzuführen und muss bei der Ergebnisinterpretation berücksichtigt werden.

\subsubsection{Zusammenhang zwischen dem Langzeitzuckerwert und Grad der Ischämie}

Der $\mathrm{HbA}_{1 c}$ zeigte bei der Schwellenwertanalyse einen deutlichen Unterschied zwischen Patienten der Gruppe G1 und G2. Bei einer FAZ-Größe kleiner oder gleich 0,35 $\mathrm{mm}^{2}$ lag der Langzeitzuckerwert bei $6,8 \%$. Bei einer FAZ-Größe größer $0,35 \mathrm{~mm}^{2}$ lag er bei $7,7 \%$. Für die Quartilanalyse zeigte sich eine entgegengesetzte Verteilung, für die sich keine Erklärung finden ließ. Die Korrelationsanalyse zeigte nur einen geringfügigen Zusammenhang ohne statistische Signifikanz.

Bezüglich des $\mathrm{HbA}_{1 c}$ existieren bereits verschiedene Studien, die einen gleichgerichteten Zusammenhang zwischen dem Schweregrad einer diabetischen Retinopathie und der Höhe der Blutzuckerwerte nahelegen (Singh et al. 1991; Long et al. 2017). Der in unserer Studie nachgewiesene positive Zusammenhang der beiden Parameter war daher absehbar. Eine lineare Verteilung der Messwerte ist jedoch unwahrscheinlich, da die aktuell geltende Leitlinie die 
Einstellung hoch-normaler Langzeitzuckerwerte für Patienten mit Diabetes mellitus vorsieht. In der ACCORD-Studie wurde belegt, dass eine zu aggressive Senkung des $\mathrm{HbA}_{1 c}$ zur Steigerung der Mortalität führt, auch wenn die Studienergebnisse heftig diskutiert werden (ACCORD Study Group et al. 2010). Die Deutsche Diabetes Gesellschaft empfiehlt derzeit für Typ-2-Diabetiker einen Langzeitzuckerwert von etwa 6,5 - 7,5\% (NVL Therapie des Typ-2-Diabetes 2013).

Die einmalige Erhebung des Langzeitzuckerwerts stellt außerdem im Hinblick auf eine meist Jahrzehnte bestehende Krankheit nur eine Momentaufnahme dar. Um verlässlichere Daten bezüglich des Einflusses des Blutzuckerspiegels zu gewinnen, wäre es sinnvoll eine Langzeitbeobachtung von Ischämiefläche und wiederholt gemessenen $\mathrm{HbA1c}$-Werten anzufertigen, wobei dies bei der geplanten einmaligen Erfassung der Studienparameter nicht möglich war (Long et al. 2017).

\subsubsection{Zusammenhang zwischen Erkrankungsdauer, Therapiedauer und dem Grad der Ischämie}

Erkrankungsdauer und Therapiedauer mit Insulin ergaben deutliche Unterschiede, obwohl sie nicht signifikant waren. In der Korrelationsanalyse bestätigte sich der Verdacht, dass die Ischämiefläche bei zunehmender Erkrankungsdauer zunahm. Für die Therapiedauer mit Insulin bestand in allen drei Analysen ein positiver Zusammenhang, also eine Zunahme der Ischämiefläche bei zunehmender Dauer der Insulintherapie, jedoch ohne statistische Signifikanz.

\subsubsection{Zusammenhang zwischen der Refraktion und dem Grad der Ischämie}

Die Erhebung der Daten bezüglich der Refraktion der untersuchten Augen stellte in erster Linie ein Merkmal zur Qualitätskontrolle der anderen untersuchten Parameter dar. Alle eingeschlossenen Augen weisen nur geringfügige Abweichung von der Norm auf, sodass der Einfluss eines hohen Refraktionsfehlers auf die Messungen der funktionellen Parameter gering ist.

Grundsätzlich dienten die Erhebung und Auswertung der demografischen Daten (Durchschnittsalter, Diabetes-Typ, Langzeitzuckerwert, Refraktion sowie Therapieform und -dauer) zur Qualitätssicherung der Studiendaten. Mithilfe der Daten wollten wir untersuchen, ob es sich bei unserer Stichprobe um eine repräsentative Gruppe handelt, und in welchem Rahmen mithilfe der erhobenen Daten der Funktionstests relevante Aussagen über den Einfluss der makulären Ischämie getroffen werden können. 


\subsection{Limitationen}

Die vorliegende Studie beinhaltet einige Limitationen, die in prospektiven Studien nicht zu verhindern sind. Als generelle Ausschlusskriterien für die Teilnahme an der Studie galten Augenerkrankungen wie retinale Gefäßverschlüsse, Glaukom, epiretinale Gliose, Makulaforamen, altersabhängige Makuladegeneration, Uveitis posterior und Vernarbungen im Bereich der Makula. Diese Erkrankungen überlagern die funktionellen Ergebnisse, sind im klinischen Alltag aber durchaus normal. Insofern spiegeln die Studienergebnisse nicht die Realität wider. Zusätzlich musste das geplante Kollektiv nochmals etwas verringert werden, was bei prospektiven Studien allerdings üblich ist. Insgesamt war der Einschluss von 40 Patienten geplant und es wurden dementsprechend 40 Patienten untersucht. Aus diesem Kollektiv mussten nachträglich vier Patienten aufgrund von fehlerhaften oder fehlenden Fluoreszeinangiografien ausgeschlossen werden, da sie sich so nicht mehr für die Datenauswertung eigneten.

Für die Studie wurden Patienten aus der Poliklinik der UMG-Augenklinik, als auch Patienten aus der UMG Diabetes-Ambulanz rekrutiert. Die Patienten, die durch ihre Anbindung an die Ambulanz für die Studie untersucht wurden, eigneten sich in besonderem Maße für die Rekrutierung, da bei einem großen Teil der Probanden keine Augenerkrankung bekannt war. Das hohe Maß an Naivität der Studienaugen bedeutete in den meisten Fällen jedoch auch geringere pathologische diabetische Veränderungen. Im Rückblick wurden im Rahmen der Patientenrekrutierung viele Patienten eingeschlossen, die zwar optimal den Einschlusskriterien entsprachen, jedoch deshalb auch wenig retinale Veränderungen aufwiesen. Das Patientenkollektiv lag deshalb nicht repräsentativ im Bereich einer Normalverteilung, sondern spiegelte einen weniger kranken Teil der Patienten mit diabetischer Retinopathie wider. Es ist fraglich, ob sich ein adäquates Patientenkollektiv untersuchen lässt, welches einen Querschnitt der an Diabetes erkrankten Bevölkerung darstellt, oder ob der Ausschluss der Patienten mit Folgeerkrankungen stets die vollständige Repräsentation dieser Kohorte limitiert.

Im Rahmen der Studie wurden die FAZ der Patienten vermessen und sowohl miteinander als auch mit anderen visuellen Parametern verglichen. Für den Bereich der FAZ ist es physiologisch, dass sich dort keine Gefäße befinden und er nicht durchblutet wird. Durch die Minderperfusion von Gefäßen im Rahmen von Erkrankungen des Augenhintergrundes kann sich dieser Bereich erweitern. Krankhaft nicht durchblutete Areale im Bereich um die eigentliche FAZ herum sind weder in der Funduskopie noch in der OCT von den physiologisch nicht perfundierten Bereichen zu unterscheiden. Allein in der Fluoreszeinangiografie lassen sich Hinweise auf weniger durchblutete Areale beispielsweise durch Veränderungen am Kapillarkranz nachweisen. Anhand der Normwerte 
für die Größe der FAZ zwischen 0,25 mm² und 0,4 mm² (John et al. 2011; Chui et al. 2014; Carpineto et al. 2016) wurden Werte größer als $0,4 \mathrm{~mm}^{2}$ als ein Zuwachs an minderperfundierter Fläche gewertet. Es konnte jedoch in Studien nachgewiesen werden, dass die Größe und auch die Form der Makula sowie der FAZ in hohem Maße von Individuum zu Individuum variiert. Sowohl Alter als auch Geschlecht scheinen einen Einfluss auf die Größe der FAZ zu haben (Ghassemi et al. 2017). Eine Norm für die Form lässt sich kaum bestimmen, ebenso schwanken Größe und Dicke sowohl in gesunden Probanden (Magrath et al. 2017) als auch in Patienten mit bekanntem Diabetes mellitus (Sim et al. 2014). Die hohe Variabilität bezogen auf Makula und Fovea macht es schwierig einem definierten Normwert in Bezug auf die Korrelationsanalyse zu folgen. 


\section{Zusammenfassung}

In der vorliegenden Studie sollte der Einfluss der makulären Ischämie auf funktionelle und morphologische retinale Parameter bei Patienten mit diabetischer Retinopathie untersucht werden. Ziel dieser Studie war es im Besonderen, Zusammenhänge zwischen den verschiedenen Untersuchungsparametern und der Größe der Ischämiefläche im Bereich der fovealen avaskulären Zone (FAZ) nachzuweisen. Hintergrund ist der Wunsch, mithilfe einer möglichen Korrelation eine verbesserte Frühdiagnostik zur Detektion der diabetischen Makulopathie etablieren zu können.

Für die Studie wurden insgesamt 40 Diabetiker unterschiedlichen Alters und Typs untersucht und 36 Patienten mit einem kompletten Datensatz in die Analyse eingeschlossen. Es wurden die Refraktion, die Sehkraft, das Kontrastsehen und die Größe des Gesichtsfeldes bestimmt. Außerdem wurden für jeden Patienten eine OCT-Untersuchung und eine Fluoreszeinangiografie durchgeführt. Jeder dieser gemessenen Parameter wurde mit der Größe der makulären Ischämie korreliert. Die Auswertung zeigt signifikante Zusammenhänge zwischen der makulären Ischämiefläche einerseits und einzelnen Parametern (Kontrastsehstärke, Größe des Gesichtsfelddefekts und totales makuläres Volumen) andererseits. Mit zunehmender makulärer Ischämie wird das Kontrastsehen schlechter und die Größe der Gesichtsfeldausfälle nimmt zu. Zudem konnte gezeigt werden, dass mit Vergrößerung der Ischämiefläche sowohl die Schichtdicke als auch das zentrale Netzhautvolumen zunehmen. Der Visus hingegen war nicht signifikant verändert.

Eine genaue Definition einer visusmindernden geschädigten Ischämiefläche war allerdings nicht möglich. Es kann demnach keine klare Grenze definiert werden, jenseits derer eine Makula mit einem unwiederbringlichen Funktionsverlust einhergeht.

Zusammenfassend konnte durch die Studie gezeigt werden, dass die Parameter Kontrastsehen, relatives Zentralskotom und makuläres Volumen in der OCT mit der makulären Ischämie korrelieren. Falls man im Rahmen eines Screenings mit funktionellen und einfachen bildmorphologischen Methoden mögliche zentral-ischämische Patienten identifizieren möchte, sind diese drei Untersuchungsmethoden geeignet und der Messung der Sehschärfe deutlich überlegen. 


\section{Anhang}

\subsection{Patienteninformation}

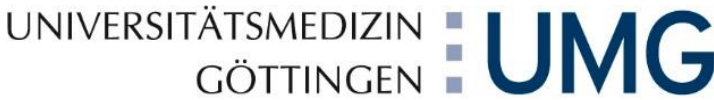

Zentrum 13 - Augenklinik mit Poliklinik

Abteilung Augenheilkunde

Direktor: Prof. Dr. med. Hans Hoerauf

Studienleiter: Dr. med. Josep Callizo

Robert-Koch-Strasse 40, 37075 Göttingen

Tel.: 0551-39 6776/ Fax: 0551-39 6787

E-mail: josep.callizo@med.uni-goettingen.de

\section{Einfluss der makulären Ischämie auf funktionelle und morphologische Parameter bei Patienten mit diabetischer Retinopathie}

\section{- Patienteninformation -}

\section{Sehr geehrte Patientin, sehr geehrter Patient,}

wir möchten Sie um die freiwillige Teilnahme an einer wissenschaftlichen Studie bitten.

Durch den bei Ihnen bestehenden Diabetes mellitus (Zuckerkrankheit) haben die kleinsten Gefäße der Netzhaut etwas gelitten. Unter der Netzhaut versteht man den sog. Fotofilm des Auges, der das Licht in Information umwandelt. Die Folge der Zuckererkrankung kann sich am Auge z.B. durch Blutungen oder Schwellungen in der Netzhaut bemerkbar machen.

Es ist aber auch möglich, dass Netzhautanteile aufgrund der zuckerbedingten Gefäßschädigung nicht mehr durchblutet werden. Falls Netzhautabschnitte einmal nicht mehr durchblutet sind, kann die Durchblutung an diesen Stellen auch nicht wieder hergestellt werden. Das ist vor allem im Zentrum der Netzhaut ungünstig, da sich hier der Hauptvorgang des Sehens abspielt.

Es gibt bisher nur sehr wenige Daten darüber, ab wann sich sauerstoffarme und nicht mehr durchblutete Abschnitte im Rahmen einer diabetischen Makulopathie auf das zentrale Sehen auswirken.

Dieses Wissen würde uns im klinischen Alltag jedoch sehr helfen, Untersuchungen und die Behandlungsmethode gezielter einzusetzen. Wir möchten Sie deshalb bitten, uns bei einer wissenschaftlichen Studie zu unterstützen, die wir bei Patienten mit einer diabetischen Makulopathie durchführen möchten. Die Teilnahme an dieser wissenschaftlichen Studie ist freiwillig. Die Entscheidung, ob Sie an dieser wissenschaftlichen Studie teilnehmen oder nicht, hat keinen Einfluss auf Ihre weitere augenärztliche Behandlung. 
Ihre Teilnahme an dieser Studie ist freiwillig. Sie werden in diese Studie also nur dann einbezogen, wenn Sie dazu schriftlich Ihre Einwilligung erklären. Sofern Sie nicht an der Studie teilnehmen oder später aus ihr ausscheiden möchten, erwachsen Ihnen daraus keine Nachteile.

Bitte lesen Sie die nachstehende Information sorgfältig. Bei Unklarheiten wird Ihr Arzt Sie ausführlich beraten.

\section{Was ist das Ziel der Studie?}

Das Ziel unserer Studie besteht darin herauszufinden, ab wann ein Sauerstoffmangel der zentralen Netzhautgefäße Auswirkungen auf Ihre Sehkraft hat.

Ein möglicher Zusammenhang hängt wahrscheinlich von mehreren Faktoren ab. Um dies herauszufinden wollen wir bestimmte Sehfunktionen (wie z.B. Sehkraft, Kontrastsehen, Gesichtsfeld) untersuchen.

\section{Was sind die Voraussetzungen für eine Teilnahme?}

Voraussetzung zur Teilnahme ist, dass bei Ihnen eine diabetische Makulopathie vorliegt und dass eine Fluoreszenzangiographie geplant ist. Nach eingehender mündlicher und schriftlicher Aufklärung über den Sinn und den Ablauf der Studie werden wir Sie bitten, schriftlich Ihr Einverständnis zur Teilnahme zu bekunden.

\section{Welche Untersuchungen werden durchgeführt?}

Es werden folgende Untersuchungen durchgeführt, die auch immer bei Patienten mit der Erkrankung durchgeführt werden:

- Befragung der Patienten zur Krankengeschichte

- Sehschärfenmessung

- OCT (Schichtaufnahme der Netzhaut)

- Netzhautfotos

- Untersuchung des vorderen und hinteren Augenabschnittes

- Augeninnendruckmessung

Folgende 2 Untersuchungen werden nicht immer und in Ihrem Fall zusätzlich im Rahmen der Studie durchgeführt: 
- Messen des Kontrastsehens. Das Kontrastsehen ist die Fähigkeit des Auges, Helligkeitsunterschiede zu erkennen. Die Prüfung des Kontrastsehens kann Auskunft darüber geben, warum das Sehen trotz guten Visus als eingeschränkt empfunden wird.

- Gesichtsfeldtest. Das Gesichtsfeld ist der Bereich, den ein Mensch bei ruhig gestellten Augen und ohne den Kopf oder den Körper zu bewegen überblicken kann. Durch Lichtpunkte unterschiedlicher Position und Helligkeit kann dieses Gesichtsfeld und damit die visuelle Sensibilität einzelner Netzhautareale geprüft werden.

Diese Untersuchungen dauern zusätzlich ca. 30 min.

\section{Wie oft werden Sie untersucht?}

Die genannten Untersuchungen werden einmalig an Ihnen durchgeführt. Es sind keine weiteren Termine erforderlich.

\section{Was sind die möglichen Risiken oder Nachteile einer Teilnahme an der Studie?}

Es gibt keine Risiken. Es handelt sich um eine Studie mit nichtinvasiven Untersuchungsverfahren.

\section{Welchen Vorteil haben Sie durch Ihre Teilnahme an der Studie?}

Eigennutzen: Durch Ihre Teilnahme an der Studie gewinnen wir Informationen über den Verlauf Ihrer diabetischen Makulopathie und können somit gegebenenfalls die Prognose optimieren.

Gruppennutzen: Die Ergebnisse der Untersuchungen könnten in Zukunft helfen, Patienten mit diabetischer Makulopathie gezielter zu behandeln.

Fremdnutzen: Durch Ihre Teilnahme helfen Sie mit, eine bestmögliche Behandlung der diabetischen Makulopathie zu gewährleisten.

\section{Was geschieht mit Ihren medizinischen Daten?}

Es gilt die ärztliche Schweigepflicht. Jede Information über Sie wird zu jeder Zeit mit der größtmöglichen Vertraulichkeit behandelt. Die medizinischen Daten und Messergebnisse werden in einer zentralen Datenbank gespeichert, die den jeweils geltenden datenschutzrechtlichen Auflagen Rechnung trägt. Ihre Daten werden pseudonymisiert. Das heißt, diese werden durch ein Pseudonym (eine mehrstellige Buchstaben- und Zahlenkombination) ersetzt, um Ihre Identität zu schützen. In keiner wissenschaftlichen Veröffentlichung wird Ihr Name erscheinen. Ihre Daten werden auf keinen Fall bekannt gegeben.

Ihre personenbezogenen Studiendaten werden für 10 Jahre gespeichert.

Sie können jederzeit, auch ohne Angabe von Gründen, Ihre Teilnahme beenden, ohne dass Ihnen dadurch irgendwelche Nachteile bei Ihrer medizinischen Behandlung entstehen.

Im Falle einer Rücknahme der Einwilligung werden auf ihren Wunsch hin die bereits erhobenen personenbezogenen Daten gelöscht. 


\subsection{Einverständniserklärung}

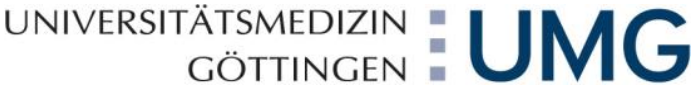

Zentrum 13 - Augenklinik mit Poliklinik

Klinik für Augenheilkunde

Direktor: Prof. Dr. med. Hans Hoerauf

Studienleiter Dr. med. Josep Callizo

Robert-Koch-Strasse 40, 37075 Göttingen

Tel.: 0551-39 6776/ Fax: 0551-39 6787

E-Mail: josep.callizo@med.uni-goettingen.de

\section{Einfluss der makulären Ischämie auf funktionelle und morphologische \\ Parameter bei Patienten mit diabetischer Retinopathie}

\section{Einwilligungserklärung}

Patientenaufkleber

Teilnehmer- $\mathrm{Nr}$

Ich bin in einem persönlichen Gespräch durch den Prüfarzt

Name der Ärztin/des Arztes

ausführlich und verständlich über Wesen, Bedeutung, Risiken und Tragweite der Untersuchung aufgeklärt worden. Ich habe darüber hinaus den Text der Patienteninformation sowie die hier nachfolgend abgedruckte Datenschutzerklärung gelesen und verstanden. Ich hatte die Gelegenheit, mit dem Prüfarzt über die Durchführung der Untersuchung zu sprechen. Alle meine Fragen wurden zufriedenstellend beantwortet.

Möglichkeit zur Dokumentation zusätzlicher Fragen seitens des Probanden oder sonstiger Aspekte des Aufklärungsgesprächs: 
Ich hatte ausreichend Zeit, mich zu entscheiden.

Mir ist bekannt, dass ich jederzeit und ohne Angabe von Gründen meine Einwilligung zur zusätzlichen Untersuchung zurückziehen kann (mündlich oder schriftlich), ohne dass mir daraus Nachteile entstehen.

\section{Datenschutz:}

Mir ist bekannt, dass bei dieser Untersuchung personenbezogene Daten, insbesondere medizinische Befunde über mich erhoben, gespeichert und ausgewertet werden sollen. Die Verwendung der Angaben über meine Gesundheit erfolgt nach gesetzlichen Bestimmungen und setzt vor der Teilnahme an der Untersuchung folgende freiwillig abgegebene Einwilligungserklärung voraus, das heißt ohne die nachfolgende Einwilligung kann ich nicht an der klinischen Prüfung teilnehmen.

1. Ich erkläre mich damit einverstanden, dass im Rahmen dieser klinischen Studie personenbezogene Daten, insbesondere Angaben über meine Gesundheit, über mich erhoben und in Papierform sowie auf elektronischen Datenträgern in der Universitäts-Augenklinik Göttingen aufgezeichnet werden.

2. Außerdem erkläre ich mich damit einverstanden, das autorisierte und zur Verschwiegenheit verpflichtete zuständigen Überwachungsbehörden in meine beim Prüfarzt vorhandenen personenbezogenen Daten, insbesondere meine Gesundheitsdaten, Einsicht nehmen, soweit dies für die Überprüfung der ordnungsgemäßen Durchführung der Studie notwendig ist. Für diese Maßnahme entbinde ich den Prüfarzt von der ärztlichen Schweigepflicht.

3. Die Einwilligung zur Erhebung und Verarbeitung meiner personenbezogenen Daten kann ich jederzeit widerrufen. Ich bin bereits darüber aufgeklärt worden, dass ich jederzeit die Teilnahme an der Studie beenden kann. Im Fall eines solchen Widerrufs meiner Einwilligung, können auf meinen Wunsch hin auch meine Daten aus der Studiensammlung gelöscht werden.

4. Ich erkläre mich damit einverstanden, dass meine Daten nach Beendigung oder Abbruch der Studie mindestens zehn Jahre aufbewahrt werden. Danach werden meine personenbezogenen Daten gelöscht, soweit nicht gesetzliche, satzungsmäßige oder vertragliche Aufbewahrungsfristen entgegenstehen (vertraglich vereinbarte Fristen müssen hier genannt werden).

5. Ich bin über folgende gesetzliche Regelung informiert: Falls ich meine Einwilligung, an der Studie teilzunehmen, widerrufe, müssen alle Stellen, die meine personenbezogenen Daten, insbesondere Gesundheitsdaten, gespeichert haben, unverzüglich prüfen, inwieweit die gespeicherten Daten für die in Nr. 3 genannten Zwecke noch erforderlich sind.

Nicht mehr benötigte Daten sind unverzüglich zu löschen.

6. Ich erkläre mich damit einverstanden, dass im Rahmen dieser Untersuchung personenbezogene Daten, Bestandteil meiner Patientenakte in der Universitäts-Augenklinik Göttingen werden.

7. Ich erkläre mich damit einverstanden, dass meine Daten ohne Hinweis auf die Person für eine Auswertung und Veröffentlichung verwendet werden können. 
Ich erkläre mich freiwillig dazu bereit, an der Studie teilzunehmen.

Ein Exemplar der Patienteninformation und -Einwilligung habe ich erhalten.

Ein Exemplar verbleibt im Prüfzentrum.

Name des Probanden in Druckbuchstaben

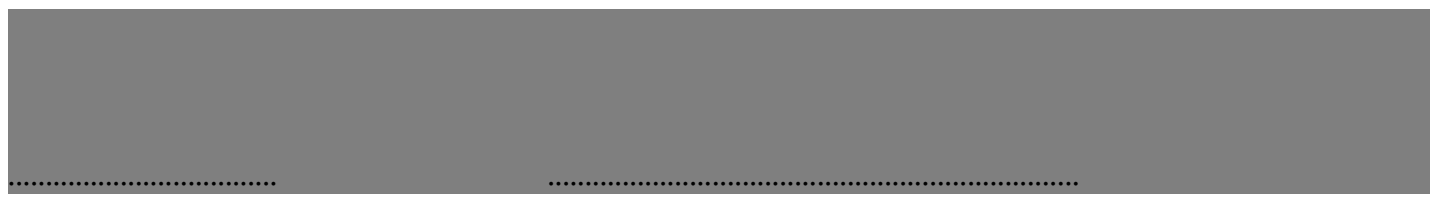

Datum

Unterschrift des Probanden 


\subsection{Untersuchungsbögen}

\subsubsection{Visual Acuity Score}

\section{UNIVERSITÄTSMEDIZIN $=1$
GÖTTINGEN $=10$ \\ Visual Acuity \\ Anleitung: Jeder richtig gelesener Buchstabe wird eingekreist. Falsche oder übersprungene Buchstaben werden nicht markiert. Nur das Studienauge wird untersuch.}

Datum der Untersuchung:

Studienauge:

rechtes

$\square$ linkes

Refraktion:

RA Sphäre:

Zylinder Achse: Rechtes Auge Korrekte

LA Sphäre: Zylinder: Chart R Anzahl

Åquivalent 4 meter

Reihe Åquivalent

4 meter

$\begin{array}{llllll}20 / 200 & H & \text { V } & \text { Z } & \text { D } & \text { S }\end{array}$

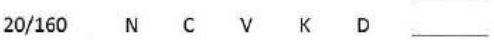

20/125 C Z S H N

20/100 O N V S R

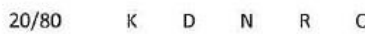

20/63 Z K C S S V

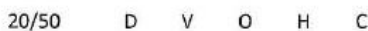

20/40 O $\quad$ H $\quad$ V $\quad$ C $\quad$ K

20/32 H $\quad$ Z $\quad C \quad K \quad$ O

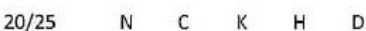

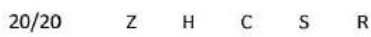

20/16 S $\quad Z \quad R \quad$ D $N$

20/12.5

$20 / 10$

$\mathrm{H}$
$\mathrm{R}$

Gesamt Anzahl korrekt gelesen bei 4 metern:* *Wenn < 3 Buchstaben bei Reihe 1, dann $1 \mathrm{~m}$ n.e. $\square$ Addiere + 0.75 Sphăre

Korrekte

Anzahl

1 meter

1
2
3
4
5
6

$\begin{array}{lllllll}20 / 800 & \text { H } & \text { V } & \text { Z } & \text { D } & \text { S } & \\ 20 / 640 & \text { N } & \text { C } & \text { V } & \text { K } & \text { D } & \\ 20 / 500 & \text { C } & \text { Z } & \text { S } & \text { H } & \text { N } & \\ 20 / 400 & \text { O } & \text { N } & \text { V } & \text { S } & \text { R } & \\ 20 / 320 & \text { K } & \text { D } & \text { N } & \text { R } & \text { O } & \\ 20 / 250 & \text { Z } & \text { K } & \text { C } & \text { S } & \text { V } & \end{array}$

Gesamt Anzahl korrekt zelesen bei 1 metern Visual Acuity Score - Rechtes Auge

A. Summe der Buchstaben 4 meter:

B. Wenn $\geq 20$, addiere 30 , sonst 0

C. . Summe der Buchstaben 1 meter, sonst 0:

Kleinste Reihe mit 3 gelesenen Buchstaben

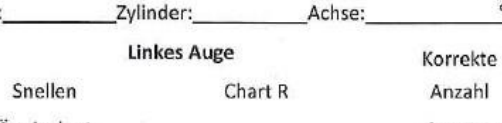

Äquivalent 4 meter

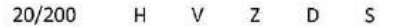

$20 / 160 \quad N \quad C \quad V \quad K \quad$ D

20/125 C $\quad$ Z $\quad S \quad H \quad$ N

20/100 O N V S R

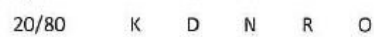

20/63 Z K C S V

20/50 D $V$ O $\quad H \quad$ C

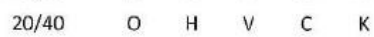

20/32 H Z C $\quad K \quad$

$20 / 25$ N $\quad C \quad$ K $\quad H \quad$ D

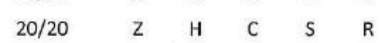

$\begin{array}{lllllll}20 / 16 & S & Z & R & D & N\end{array}$

$20 / 12.5$

20/10

$\longrightarrow$

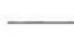

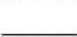

$\longrightarrow$

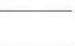

esamt Anzahl korrekt gelesen bei 4 metern:* *Wenn < 3 Buchstaben bei Reihe 1, dann $1 \mathrm{~m}$
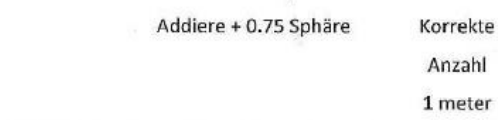

$\begin{array}{lllllll}20 / 800 & \text { H } & \text { V } & \text { Z } & \text { D } & \text { S } & \\ 20 / 640 & \text { N } & \text { C } & \text { V } & \text { K } & \text { D } & \\ 20 / 500 & \text { C } & \text { Z } & \text { S } & \text { H } & \text { N } & \\ 20 / 400 & \text { O } & \text { N } & \text { V } & \text { S } & \text { R } & \\ 20 / 320 & \text { K } & \text { D } & \text { N } & \text { R } & \text { O } & \\ 20 / 250 & \text { Z } & \text { K } & \text { C } & \text { S } & \text { V } & \end{array}$

Gesamt Anzahl korrekt gelesen bei 1 metern: Visual Acuity Score - Linkes Auge

A. Summe der Buchstaben 4 meter:

B. Wenn $\geq 20$, addiere 30 , sonst 0

C. . Summe der Buchstaben 1 meter, sonst 0 :

Kleinste Reihe mit 3 gelesenen Buchstaben

Rechtes Auge: Summe von A, B und C

Linkes Auge: Summe von A, B und C 


\subsubsection{Bailey-Lovie-Chart}

Name:

Datum:

Visite:

Studienauge:

\begin{tabular}{|c|c|l|l|}
\hline \multicolumn{5}{|c|}{ OD } \\
\hline Snellen & Dezimal & \multicolumn{2}{|c|}{ High Contrast } \\
\hline $20 / 200$ & 0,1 & R K U P F & \\
\hline $20 / 160$ & 0,125 & B V H E N & \\
\hline $20 / 125$ & 0,16 & U F P K D & \\
\hline $20 / 100$ & 0,2 & E N V H R & \\
\hline $20 / 80$ & 0,25 & P K F D B & \\
\hline $20 / 63$ & 0,32 & V R H N U & \\
\hline $20 / 50$ & 0,4 & K B D F E & \\
\hline $20 / 40$ & 0,5 & H N U R P & \\
\hline $20 / 32$ & 0,625 & F B E D V & \\
\hline $20 / 25$ & 0,8 & N R U P K & \\
\hline $20 / 20$ & 1 & D B E V H & \\
\hline & & & \\
\hline
\end{tabular}

\begin{tabular}{|c|c|}
\hline OD & \\
\hline Low Cont & ast \\
\hline$F \cup H E P$ & \\
\hline $\mathrm{K} B \vee \mathrm{R} \mathrm{N}$ & \\
\hline D H U F E & \\
\hline$P R K B V$ & \\
\hline $\mathrm{N} D \mathrm{~F} \cup \mathrm{H}$ & \\
\hline E B K P R & \\
\hline VNDFU & \\
\hline H E P K B & \\
\hline$R D N \vee F$ & \\
\hline U H P E K & \\
\hline $\mathrm{B} \vee \mathrm{F} \mathrm{N}$ & \\
\hline & \\
\hline
\end{tabular}

\begin{tabular}{|c|c|l|l|}
\hline \multicolumn{5}{|c|}{ OS } \\
\hline Snellen & Dezimal & \multicolumn{3}{|c|}{ High Contrast } \\
\hline $20 / 200$ & 0,1 & R K U P F & \\
\hline $20 / 160$ & 0,125 & B V H N & \\
\hline $20 / 125$ & 0,16 & U F P K D & \\
\hline $20 / 100$ & 0,2 & E N V H R & \\
\hline $20 / 80$ & 0,25 & P K F D B & \\
\hline $20 / 63$ & 0,32 & V R H N U & \\
\hline $20 / 50$ & 0,4 & K B D F E & \\
\hline $20 / 40$ & 0,5 & H N U R P & \\
\hline $20 / 32$ & 0,625 & F B E D V & \\
\hline $20 / 25$ & 0,8 & N R U P K & \\
\hline $20 / 20$ & 1 & D B E V H & \\
\hline & & & \\
\hline
\end{tabular}

\begin{tabular}{|c|c|}
\hline OS & \\
\hline Low Cont & \\
\hline$F \cup H E P$ & \\
\hline $\mathrm{K} B \vee \mathrm{R} \mathrm{N}$ & \\
\hline D H U F E & \\
\hline$P R K B V$ & \\
\hline$N D F U H$ & \\
\hline E B K P R & \\
\hline$V N D F U$ & \\
\hline H E P K B & \\
\hline$R D N \vee F$ & \\
\hline U H P E K & \\
\hline$B \vee F N D$ & \\
\hline & \\
\hline
\end{tabular}




\section{Literaturverzeichnis}

Acan D, Karahan E, Kocak N, Kaynak S (2018): Evaluation of systemic risk factors in different optical coherence tomographic patterns of diabetic macular edema. Int J Ophthalmol 11, 1204-1209

ACCORD Study Group, ACCORD Eye Study Group, Chew EY, Ambrosius WT, Davis MD, Danis RP, Gangaputra S, Greven CM, Hubbard L, Esser BA et al. (2010): Effects of medical therapies on retinopathy progression in type 2 diabetes. N Engl J Med 363, 233-244

Ahmadpour-Baghdadabad M, Manaviat M, Shojaoddiny-Ardekani A (2013): Optical coherence tomography in diabetic macular edema: patterns and related risk factors. Nepal J Ophthalmol $\underline{5}, 190-194$

Antonetti DA, Barber AJ, Hollinger LA, Wolpert EB, Gardner TW (1999): Vascular endothelial growth factor induces rapid phosphorylation of tight junction proteins occludin and zonula occluden 1. A potential mechanism for vascular permeability in diabetic retinopathy and tumors. J Biol Chem 274, 2346323467

Antonetti DA, Klein R, Gardner TW (2012): Diabetic retinopathy. N Engl J Med 366 1227-1239

Avogaro A, Albiero M, Menegazzo L, de Kreutzenberg S, Fadini GP (2011): Endothelial dysfunction in diabetes. Diabetes Care 34, S.285-S.290

Bach M, Wesemann W, Kolling G, Bühren J, Krastel H, Schiefer U (2008): Photopisches Kontrastsehen: Örtliche Kontrastempfindlichkeit. Ophthalmol $\underline{105}, 46-59$

Bain SC, Klufas MA, Ho A, Matthews DR (2019): Worsening of diabetic retinopathy with rapid improvement in systemic glucose control: A review. Diabetes Obes Metab 21, 454-466

Beulens JWJ, Patel A, Vingerling JR, Cruickshank JK, Hughes AD, Stanton A, Lu J, McG Thom SA, Grobbee DE, Stolk RP et al. (2009): Effects of blood pressure lowering and intensive glucose control on the incidence and progression of retinopathy in patients with type 2 diabetes mellitus: a randomised controlled trial. Diabetologia 52, 2027-2036

Bressler NM, Edwards AR, Antoszyk AN, Beck RW, Browning DJ, Ciardella AP, Danis RP, Elman MJ, Friedman SM, Glassman AR et al. (2008): Retinal thickness on stratus optical coherence tomography ${ }^{\mathrm{TM}}$ in people with diabetes and minimal or no diabetic retinopathy. Am J Ophthalmol 145, 894-901

Broe R, Rasmussen ML, Frydkjaer-Olsen U, Olsen BS, Mortensen HB, Peto T, Grauslund J (2014): The 16-year incidence, progression and regression of diabetic retinopathy in a young population-based Danish cohort with type 1 diabetes mellitus: The Danish cohort of pediatric diabetes 1987 (DCPD1987). Acta Diabetol 51, 413-420

Bundesärztekammer-Leitlinie 2015 zu Prävention und Therapie von Netzhautkomplikationen bei Diabetes Kurzfassung, 2. Auflage. Version 2. 2015.

Byeon SH, Chu YK, Lee H, Lee SY, Kwon OW (2009): Foveal ganglion cell layer damage in ischemic diabetic maculopathy: correlation of optical coherence tomographic and anatomic changes. Ophthalmology $\underline{116}, 1949-1959$

Carpineto P, Mastropasqua R, Marchini G, Toto L, di Nicola M, di Antonio L (2016): Reproducibility and repeatability of foveal avascular zone measurements in healthy subjects by optical coherence tomography angiography. $\mathrm{Br} \mathrm{J}$ Ophthalmol $\underline{100}$ 671-676 
Chan A, Duker JS, Ko TH, Fujimoto JG, Schuman JS (2006): Normal macular thickness measurements in healthy eyes using stratus optical coherence tomography. Arch Ophthalmol 124, 193-198

Chew EY, Klein ML, Ferris FL, Remaley NA, Murphy RP, Chantry K, Hoogwerf BJ, Miller D (1996): Association of elevated serum lipid levels with retinal hard exudate in diabetic retinopathy. Early Treatment Diabetic Retinopathy Study (ETDRS) Report 22. Arch Ophthalmol $1960 \underline{114}$ 1079-1084

Choi JA, Kim HW, Kwon JW, Shim Y, Jee DH, Yun JS, Ahn YB, Park CK, Ko SH (2017): Early inner retinal thinning and cardiovascular autonomic dysfunction in type 2 diabetes. PLoS One 12(3): e0174377

Chui TYP, VanNasdale DA, Elsner AE, Burns SA (2014): The association between the foveal avascular zone and retinal thickness. Invest Ophthalmol Vis Sci 55, 6870-6877

Cohen SR, Gardner TW (2016): Diabetic retinopathy and diabetic macular edema. Dev Ophthalmol 55, 137-146

Davis MD, Fisher MR, Gangnon RE, Barton F, Aiello LM, Chew EY, Ferris FL, Knatterud GL (1998): Risk factors for high-risk proliferative diabetic retinopathy and severe visual loss: Early Treatment Diabetic

Retinopathy Study Report \#18. Invest Ophthalmol Vis Sci 39, 233-252

Diabetes Control and Complications Trial Research Group, Nathan DM, Genuth S, Lachin J, Cleary P, Crofford O, Davis M, Rand L, Siebert C (1993): The effect of intensive treatment of diabetes on the development and progression of long-term complications in insulin-dependent diabetes mellitus. N Engl J Med 329, 977-986

Diabetic Retinopathy Clinical Research Network (2009): Three-year follow up of a randomized trial comparing focal/grid photocoagulation and intravitreal triamcinolone for diabetic macular edema. Arch Ophthalmol $127,245-251$

Diaz M, Kevashamurthy R, Chalam KV, Grover S (2012): Normative data for macular volume by spectral-domain optical coherence tomography (Spectralis). Invest Ophthalmol Vis Sci 53, 4075-4075

Ding J, Wong TY (2012): Current epidemiology of diabetic retinopathy and diabetic macular edema. Curr Diab Rep 12, 346-354

Do DV, Nguyen QD, Boyer D, Schmidt-Erfurth U, Brown DM, Vitti R, Berliner AJ, Gao B, Zeitz O, Ruckert R et al. (2012): One-year outcomes of the da Vinci Study of VEGF Trap-Eye in eyes with diabetic macular edema. Ophthalmology $119,1658-1665$

Do DV, Wang X, Vedula SS, Marrone M, Sleilati G, Hawkins BS, Frank RN (2015): Blood pressure control for diabetic retinopathy. Cochrane Database Syst Rev. 2015(1): CD006127

Duckworth W, Abraira C, Moritz T, Reda D, Emanuele N, Reaven PD, Zieve FJ, Marks J, Davis SN, Hayward R et al. (2009): Glucose control and vascular complications in veterans with type 2 diabetes. N Engl J Med 360, 129-139

Evans JR, Michelessi M, Virgili G (2014): Laser photocoagulation for proliferative diabetic retinopathy. Cochrane Database Syst Rev. 2014(11): CD011234

Feldman-Billard S, Larger É, Massin P, Standards for screeningand surveillance of ocular complications in people with diabetes SFD study group (2018): Early worsening of diabetic retinopathy after rapid improvement of blood glucose control in patients with diabetes. Diabetes Metab $\underline{44}, 4-14$

Ferris FL, Nathan DM (2016): Preventing Diabetic Retinopathy Progression. Ophthalmology $\underline{123}$, 1840-1842

Ghassemi F, Mirshahi R, Bazvand F, Fadakar K, Faghihi H, Sabour S (2017): The quantitative measurements of foveal avascular zone using optical coherence tomography angiography in normal volunteers. J Curr Ophthalmol 29, 293-299 
Group TDR (2015): Intensive diabetes therapy and ocular surgery in type 1 diabetes. N Engl J Med $\underline{372,1722-}$ 1733

Hadjadj S, Duly-Bouhanick B, Bekherraz A, Brldoux F, Gallois Y, Mauco G, Ebran J, Marre M (2004): Serum triglycerides are a predictive factor for the development and the progression of renal and retinal complications in patients with type 1 diabetes. Diabetes Metab $\underline{30}$ 43-51

Hammes HP, Kerner W, Hofer S, Kordonouri O, Raile K, Holl RW, DPV-Wiss Study Group (2011): Diabetic retinopathy in type 1 diabetes-a contemporary analysis of 8,784 patients. Diabetologia $\underline{54}, 1977-1984$

Hammes H-P, Welp R, Kempe H-P, Wagner C, Siegel E, Holl RW, DPV Initiative-German BMBF Competence Network Diabetes Mellitus (2015): Risk factors for retinopathy and DME in type 2 diabetes-results from the German/Austrian DPV database. PLoS One 15(10): e0132492

Haritoglou C, Kernt M, Wolf A (2015): Diabetic maculopathy. Ophthalmol Z Dtsch Ophthalmol Ges $112,871-$ 883

Hee MR, Puliafito CA, Duker JS, Reichel E, Coker JG, Wilkins JR, Schuman JS, Swanson EA, Fujimoto JG (1998): Topography of diabetic macular edema with optical coherence tomography. Ophthalmology $\underline{105}$ 360-370

Heidemann C, Du Y, Schubert I, Rathmann W, Scheidt-Nave C (2013): Prävalenz und zeitliche Entwicklung des bekannten Diabetes mellitus: Ergebnisse der Studie zur Gesundheit Erwachsener in Deutschland (DEGS1). Bundesgesundheitsblatt - Gesundheitsforschung - Gesundheitsschutz $\underline{56}$, 668-677

Hemmingsen B, Lund SS, Gluud C, Vaag A, Almdal T, Hemmingsen C, Wetterslev J (2011): Targeting intensive glycaemic control versus targeting conventional glycaemic control for type 2 diabetes mellitus. Cochrane Database Syst Rev. 2011 Jun 15(6): CD008143

Hermann JM, Hammes H-P, Rami-Merhar B, Rosenbauer J, Schütt M, Siegel E, Holl RW, Mellitus on behalf of the DI the GBCND (2014): HbA1c variability as an independent risk factor for diabetic retinopathy in type 1 diabetes: A German/Austrian multicenter analysis on 35,891 Patients. PLoS One 2014; 9(3): e91137

Hooymans JM, Ballegooie EV, Schweitzer NM, Doorebos H, Reitsma WD, Slutter WJ (1982): Worsening of diabetic retinopathy with strict control of blood sugar. Lancet Lond Engl 2, 438

John D, Kuriakose T, Devasahayam S, Braganza A (2011): Dimensions of the foveal avascular zone using the Heidelberg retinal angiogram-2 in normal eyes. Indian J Ophthalmol $\underline{59}$, 9-11

Katz J, Sommer A, Witt K (1991): Reliability of Visual Field Results over Repeated Testing. Ophthalmology $\underline{98}$ 70-75

Kawasaki R, Tanaka S, Tanaka S, Yamamoto T, Sone H, Ohashi Y, Akanuma Y, Yamada N, Yamashita H, Japan Diabetes Complications Study Group (2011): Incidence and progression of diabetic retinopathy in Japanese adults with type 2 diabetes: 8 year follow-up study of the Japan diabetes complications study (JDCS). Diabetologia 54, 2288-2294

Klein R, Klein BE, Moss SE, Davis MD, DeMets DL (1984): The Wisconsin epidemiologic study of diabetic retinopathy. II. Prevalence and risk of diabetic retinopathy when age at diagnosis is less than 30 years. Arch Ophthalmol 960 102, 520-526

Klein R, Sharrett AR, Klein BEK, Moss SE, Folsom AR, Wong TY, Brancati FL, Hubbard LD, Couper D, ARIC Group (2002): The association of atherosclerosis, vascular risk factors, and retinopathy in adults with diabetes: the atherosclerosis risk in communities study. Ophthalmology $\underline{109}, 1225-1234$ 
Klein R, Marino EK, Kuller LH, Polak JF, Tracy RP, Gottdiener JS, Burke GL, Hubbard LD, Boineau R (2002): The relation of atherosclerotic cardiovascular disease to retinopathy in people with diabetes in the Cardiovascular Health Study. Br J Ophthalmol 흐, 84-90

Koleva-Georgieva DN, Sivkova NP (2010): Optical coherence tomography for the detection of early macular edema in diabetic patients with retinopathy. Folia Med (Plovdiv) $\underline{52}, 40-48$

Kozak I, Oster SF, Cortes MA, Dowell D, Hartmann K, Kim JS, Freeman WR (2011): Clinical evaluation and treatment accuracy in diabetic macular edema using navigated laser photocoagulator NAVILAS. Ophthalmology $118,1119-1124$

Lachenmayr B, Friedburg D, Hartmann E, Buser A: Physiologische Optik. In: Auge - Brille - Refraktion. 4. Auflage; Georg Thieme Verlag 2006, 14

Lattanzio R, Brancato R, Pierro L, Bandello F, laccher B, Fiore T, Maestranzi G (2002): Macular thickness measured by optical coherence tomography (OCT) in diabetic patients. Eur J Ophthalmol $12,482-487$

Lee WJ, Sobrin L, Lee MJ, Kang MH, Seong M, Cho H (2014): The relationship between diabetic retinopathy and diabetic nephropathy in a population-based study in Korea (KNHANES V-2, 3). Invest Ophthalmol Vis Sci $55,6547-6553$

Lightman S, Towler HM (2003): Diabetic retinopathy. Clin Cornerstone $\underline{5}, 12-21$

Liu T, Hu AY, Kaines A, Yu F, Schwartz SD, Hubschman JP (2011): A pilot study of normative data for macular thickness and volume measurements using cirrus high-definition optical coherence tomography. Retina Phila Pa 31, 1944-1950

Long M, Wang C, Liu D (2017): Glycated hemoglobin A1C and vitamin D and their association with diabetic retinopathy severity. Nutr Diabetes 2017 12;7(6): e281

Madonna R, Balistreri CR, Geng Y-J, De Caterina R (2017): Diabetic microangiopathy: Pathogenetic insights and novel therapeutic approaches. Vascul Pharmacol $90,1-7$

Magrath GN, Say EAT, Sioufi K, Ferenczy S, Samara WA, Shields CL (2017): Variability in foveal avascular zone and capillary density using optical coherence tomography angiography machines in healthy eyes. Retina Phila Pa 37, 2102-2111

Mallika P, Tan A, S A, T A, Alwi SS, Intan G (2010): Diabetic retinopathy and the effect of pregnancy. Malays Fam Physician Off J Acad Fam Physicians Malays $\underline{5}, 2-5$

Massin P, Erginay A, Haouchine B, Mehidi AB, Paques M, Gaudric A (2002): Retinal thickness in healthy and diabetic subjects measured using optical coherence tomography mapping software. Eur J Ophthalmol 12. $102-108$

Massin P, Bandello F, Garweg JG, Hansen LL, Harding SP, Larsen M, Mitchell P, Sharp D, Wolf-Schnurrbusch UEK, Gekkieva M et al. (2010): Safety and efficacy of ranibizumab in diabetic macular edema (RESOLVE Study): a 12-month, randomized, controlled, double-masked, multicenter phase II study. Diabetes Care 33, 2399-2405

Mathew C, Yunirakasiwi A, Sanjay S (2015): Updates in the management of diabetic macular edema. J Diabetes Res $\underline{2015}$

Mottl AK, Pajewski N, Fonseca V, Ismail-Beigi F, Chew E, Ambrosius WT, Greven C, Schubart U, Buse J (2014): The degree of retinopathy is equally predictive for renal and macrovascular outcomes in the ACCORD Trial. J Diabetes Complications 28, 874-879 
Nguyen QD, Shah SM, Khwaja AA, Channa R, Hatef E, Do DV, Boyer D, Heier JS, Abraham P, Thach AB et al. (2010): Two-year outcomes of the ranibizumab for edema of the macula in diabetes (READ-2) study. Ophthalmology $117,2146-2151$

Patel V, Rassam S, Newsom R, Wiek J, Kohner E (1992): Retinal blood flow in diabetic retinopathy. BMJ 305 , 678-683

Patelli F, Radice P, Giacomotti E (2014): Diabetic macular edema. Dev Ophthalmol 54, 164-173

Pokharel A, Shrestha GS, Shrestha JB (2016): Macular thickness and macular volume measurements using spectral domain optical coherence tomography in normal Nepalese eyes. Clin Ophthalmol 10, $511-$ 519

Popescu T, Moţa M (2009): Dyslipidemia and hypertension in patients with type 2 diabetes and retinopathy. Romanian J Intern Med Rev Roum Med Interne 47, 235-241

Ramsey DJ, Arden GB (2015): Hypoxia and Dark Adaptation in Diabetic Retinopathy: Interactions, Consequences, and Therapy. Curr Diab Rep $\underline{15}, 118$

Raum P, Lamparter J, Ponto KA, Peto T, Hoehn R, Schulz A, Schneider A, Wild PS, Pfeiffer N, Mirshahi A (2015): Prevalence and cardiovascular associations of diabetic retinopathy and maculopathy: Results from the Gutenberg Health Study. PLoS One 10(9): e0139527

Rema M, Srivastava BK, Anitha B, Deepa R, Mohan V (2006): Association of serum lipids with diabetic retinopathy in urban South Indians - the Chennai Urban Rural Epidemiology Study (CURES) Eye Study-2. Diabet Med J Br Diabet Assoc 23, 1029-1036

Sachdev N, Sahni A (2010): Association of systemic risk factors with the severity of retinal hard exudates in a north Indian population with type 2 diabetes. J Postgrad Med 56, 3-6

Saif A, Karawya S, Abdelhamid A (2014): Blood pressure is a risk factor for progression of diabetic retinopathy in normotensive patients with type 2 diabetes: correlation with carotid intima-media thickness. Endocr Regul 48, 189-194

Schmidt-Erfurth U, Lang GE, Holz FG, Schlingemann RO, Lanzetta P, Massin P, Gerstner O, Bouazza AS, Shen H, Osborne A et al. (2014): Three-year outcomes of individualized ranibizumab treatment in patients with diabetic macular edema: the RESTORE extension study. Ophthalmology $\underline{121}$ 1045-1053

Sim DA, Keane PA, Rajendram R, Karampelas M, Selvam S, Powner MB, Fruttiger M, Tufail A, Egan CA (2014): Patterns of peripheral retinal and central macula ischemia in diabetic retinopathy as evaluated by ultra-widefield fluorescein angiography. Am J Ophthalmol 158, 144-153

Simó R, Hernández C (2008): Intravitreous anti-VEGF for diabetic retinopathy: hopes and fears for a new therapeutic strategy. Diabetologia 51, 1574-1580

Singh R, Prakash V, Shukla PK, Gautam S, Maurya OP (1991): Glycosylated hemoglobin and diabetic retinopathy. Ann Ophthalmol 23, 308-311

Stratton IM, Kohner EM, Aldington SJ, Turner RC, Holman RR, Manley SE, Matthews DR (2001): UKPDS 50: risk factors for incidence and progression of retinopathy in Type II diabetes over 6 years from diagnosis. Diabetologia $\underline{44}, 156-163$

Sun T, Zhang M (2012): Characters of contrast sensitivity in diabetic patients without diabetic retinopathy. Zhonghua Yan Ke Za Zhi Chin J Ophthalmol 48, 41-46

Uçgun NI, Yildirim Z, Kiliç N, Gürsel E (2007): The importance of serum lipids in exudative diabetic macular edema in type 2 diabetic patients. Ann N Y Acad Sci 1100, 213-217 
UK Prospective Diabetes Study (UKPDS) Group (1998): Intensive blood-glucose control with sulphonylureas or insulin compared with conventional treatment and risk of complications in patients with type 2 diabetes (UKPDS 33). The Lancet 352, 837-853

van Dijk HW, Verbraak FD, Kok PHB, Garvin MK, Sonka M, Lee K, DeVries JH, Michels RPJ, van Velthoven MEJ, Schlingemann RO, Abràmoff MD (2010): Decreased retinal ganglion cell layer thickness in patients with type 1 diabetes. Invest Ophthalmol Vis Sci $\underline{51}$, 3660-3665

Voigt M, Schmidt S, Lehmann T, Köhler B, Kloos C, Voigt UA, Meller D, Wolf G, Müller UA, Müller N (2018): Prevalence and progression rate of diabetic retinopathy in type 2 diabetes patients in correlation with the duration of diabetes. Exp Clin Endocrinol Diabetes Off J Ger Soc Endocrinol Ger Diabetes Assoc $\underline{126}, 570-576$

Yau JWY, Rogers SL, Kawasaki R, Lamoureux EL, Kowalski JW, Bek T, Chen S-J, Dekker JM, Fletcher A, Grauslund $J$ et al. (2012): Global prevalence and major risk factors of diabetic retinopathy. Diabetes Care $\underline{35}$, $556-564$

Zhang J, Wang Y, Li L, Zhang R, Guo R, Li H, Han Q, Teng G, Liu F (2018): Diabetic retinopathy may predict the renal outcomes of patients with diabetic nephropathy. Ren Fail $\underline{40}$, 243-251 


\section{Danksagung}

An dieser Stelle möchte ich mich bei allen Menschen bedanken, die zur Entstehung sowie dem Abschluss dieser Arbeit beigetragen und mich auf dem Weg dorthin unterstützt haben.

Zu allererst bedanke ich mich bei meinem Doktorvater Prof. Dr. med. Nicolas Feltgen, der mir die Möglichkeit gegeben hat in der Abteilung für Augenheilkunde an diesem Projekt mitzuarbeiten. Mein besonderer Dank richtet sich in diesem Zuge außerdem an Dr. Josep Callizo, der mir als mein direkter Betreuer und Ansprechpartner stets mit Rat und Tat zur Seite stand. Mit seiner Hilfe und Unterstützung über den gesamten Weg der Entstehung dieser Arbeit war es mir möglich diese Studie umzusetzen. Er hatte bei jeglicher Fragegestellung stets ein offenes Ohr und ich habe durch unsere Zusammenarbeit viel gelernt.

Weiterhin möchte ich mir bei allen Mitarbeitern der Augenklinik Göttingen bedanken, die an der Durchführung dieser Studie mitgewirkt haben. Besonderer Dank richtet sich an dieser Stelle an das ärztliche Personal der Poliklinik, welches sich stets dafür einsetzte die Studienpatienten im fortlaufenden Betrieb in der Ambulanz zusätzlich aufzunehmen und zu untersuchen. Außerdem bedanke ich mir bei Marion Pieper, die als Study Nurse sämtliche Patienten dieser Studie betreute und, neben ihrer Arbeit, für einen reibungslosen Untersuchungsablauf sorgte. Außerdem danke ich den Mitarbeitern und Mitarbeiterinnen der Leitstelle der Poliklinik, die mir bei der Terminkoordination und Betreuung der Studienpatienten zur Seite standen.

Zusätzlicher Dank geht an Dr. Katja Gollisch für die tolle Zusammenarbeit von Augenklinik und Diabetes-Ambulanz bei der Rekrutierung der Studienpatienten sowie für die Zeit, die sie für die zusätzlichen Patientengespräche und Aktenarbeit aufgebracht hat.

Ein besonderes Dankeschön richte ich außerdem an Sebastian Pfeiffer, der mir mit seinem statistischen Fachwissen bei jeglichen Fragestellungen zur Seite stand und mir bei der Auswertung der Ergebnisse eine große Hilfe war. 